Check for updates

Cite this: RSC Adv., 2020, 10, 36413

Received 20th August 2020

Accepted 14th September 2020

DOI: 10.1039/dOra07160a

rsc.li/rsc-advances

\title{
Anisotropic quasi-one-dimensional layered transition-metal trichalcogenides: synthesis, properties and applications $\uparrow$
}

\begin{abstract}
Abhinandan Patra and Chandra Sekhar Rout (D) *
The strong in-plane anisotropy and quasi-1D electronic structures of transition-metal trichalcogenides $\left(M X_{3} ; M=\right.$ group IV or $V$ transition metal; $X=S$, Se, or Te) have pronounced influence on moulding the properties of $M X_{3}$ materials. In particular, the infinite trigonal $M X_{6}$ prismatic chains running parallel to the $b$-axis are responsible for the manifestation of anisotropy in these materials. Several marvellous properties, such as inherent electronic, optical, electrical, magnetic, superconductivity, and charge density wave (CDW) transport properties, make transition-metal trichalcogenides (TMTCs) stand out from other 2D materials in the fields of nanoscience and materials science. In addition, with the assistance of pressure, temperature, and tensile strain, these materials and their exceptional properties can be tuned to a superior extent. The robust anisotropy and incommensurable properties make the $M_{X_{3}}$ family fit for accomplishing quite a lot of compelling applications in the areas of field effect transistors (FETs), solar and fuel cells, lithium-ion batteries, thermoelectricity, etc. In this review article, a precise audit of the distinctive crystal structures, static and dynamic properties, efficacious synthesis schemes, and enthralling applications of quasi-1D $M X_{3}$ materials is made.
\end{abstract}

\section{Introduction}

The many pioneering studies based on the modern miracle material graphene ${ }^{1}$ paved the way for other monolayered and fewlayered two-dimensional transition metal dichalcogenides (TMDCs) to be studied. They gained huge attention due to their Centre for Nano and Material Sciences, Jain University, Jain Global Campu Jakkasandra, Ramanagaram, Bangalore-562112, India. E-mail: r.chandrasekhar@ jainuniversity.ac.in; csrout@gmail.com

$\dagger$ Electronic supplementary information (ESI) available. See DOI: 10.1039/d0ra07160a

strong tunable mechanical, electrical, optical, and

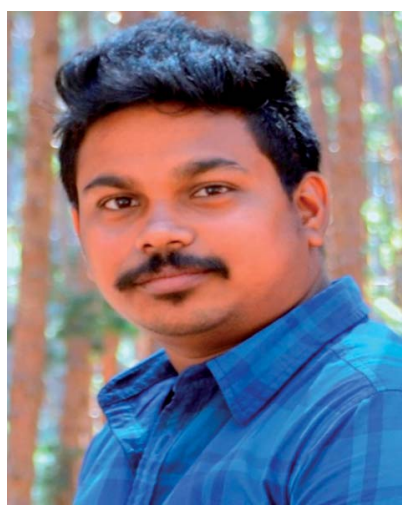

Abhinandan Patra received his M.Sc. degree in Physics from the College of Engineering and Technology under Biju Patnaik University of Technology (BPUT), Odisha, India, in 2019. He is presently pursuing his PhD degree at the Centre for Nano and Material Sciences, Jain University. His main research interests are the synthesis and comprehensive characterization of novel functionalized layered $2 D$ nanomaterials and their composites for electrochemical storage and conversion applications.

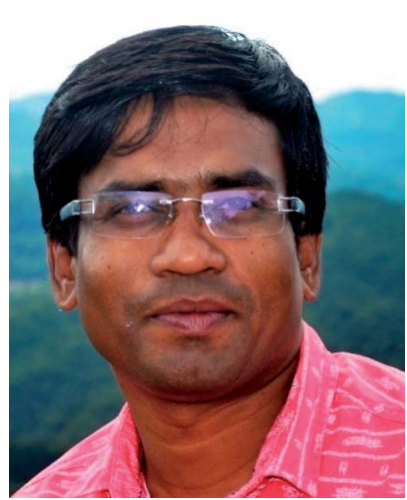

Dr Chandra Sekhar Rout is Associate Professor at the Centre for Nano \& Material Sciences, Jain University. Before joining CNMS, he was a DSTRamanujan Fellow at IIT Bhubaneswar, India (2013-2017). He received his B.Sc. (2001) and M.Sc. (2003) degrees from Utkal University and his $P h D$ from JNCASR, Bangalore (2008) under the supervision of Prof. C. N. R. Rao. He did postdoctoral research at National University of Singapore (20082009), Purdue University, USA (2010-2012), and UNIST, South Korea (2012-2013). His research interests include $2 D$ materials for sensors, supercapacitors and energy storage devices, field emitters, and electronic devices. 
physiochemical properties originating from the low dimensionality and quantum confinement effects. In accordance with the above excellent properties, they are useful for various optoelectronic and energy storage applications. Another noteworthy family of two-dimensional materials is transition metal trichalcogenides $\left(\mathrm{MX}_{3}\right)$, which have been a source of attraction for a couple of years. These $\mathrm{MX}_{3}$ materials possess typical electrical, optical, magnetic, and physical properties that are quite anisotropic. Therefore, linking the benefits of 2D materials (elasticity, robustness, ease of synthesis, and huge surface to volume ratios) with excellent quasi-one-dimensional (1D) properties can be reasonably fruitful for application in the unexplored directions of nanoelectronics and nanotechnology.

The weak van der Waals amalgamated crystal structures of $\mathrm{MX}_{3}$ architecturally and chemically constitute a very distinct family of compounds, where $\mathrm{M}$ (group IV, V, or VI) represents a metal atom and $\mathrm{X}$ is a chalcogen atom (S, Se, or Te), and the $\mathrm{M}-\mathrm{X}$ bond is ioniccovalent in nature..$^{6-9}$ The compact in-plane anisotropy and quasi one-dimensional properties of monolayer and few-layer $\mathrm{MX}_{3}$ contribute a huge amount to configuring every single property of these materials. The prismatic $\mathrm{MX}_{6}$ chains of $\mathrm{MX}_{3}$ are extended along the $b$-axis to provide strong anisotropy. Pressure, temperature, and tensile strain can tune the electronic properties, and charge density wave transportation plays a pivotal role in making metal trichalcogenides a prime material of interest in the nanoscience and materials science communities. Instead of bulk or layered $\mathrm{MX}_{3}$, monolayer metal trichalcogenides are of considerable interest in both experimental and theoretical investigations. Monolayer $\mathrm{TiS}_{3}$ and $\mathrm{TaS}_{3}$, fabricated through mechanical exfoliation, show amazing superconductivity and charge density wave phenomena. This breakthrough accounts for their pronounced, unparalleled, and plentiful applications in the field of nanoelectronics.

Wide amounts of research have been ongoing concerning the flexible fabrication of $\mathrm{MX}_{3}$ materials, and attempts to shed several layers to obtain monolayers have also been made in the past few years through many emerging top-down synthesis methods. These new-fangled methods include chemical vapour deposition (CVD), chemical vapour transport (CVT), solvothermal methods, mechanical and chemical exfoliation, etc. As far as this review is concerned, we report cumulative information regarding crystal structures, elementary properties, synthesis procedures, applications, and experimental and theoretical developments related to $\mathrm{MX}_{3}$-based materials. Firstly, emblematic crystal structures and typical electrical, optical, magnetic, and CDW transport property characteristics are thoroughly discussed. Secondly, the fabrication of these materials is looked into. Lastly, the wide variety of applications of $\mathrm{MX}_{3}$, including field emission transistors, ${ }^{\mathbf{1 2 4 - 1 2 6 , 1 4 2}}$ solar and fuel cells, ${ }^{139,143-148}$ photo-detectors and -sensors, ${ }^{33,139,150,151}$ lithium ion batteries, ${ }^{134,152-155}$ and thermoelectricity, ${ }^{156-161}$ are discussed with proper reference to previous studies.

\section{Classification and crystal structures of $\mathrm{MX}_{3}$}

Layered $\mathrm{MX}_{3}$ materials have emerged as a modern development with interesting electrical and optical properties due to their in- plane anisotropy. ${ }^{2-4}$ With robust structural in-plane anisotropy, $\mathrm{MX}_{3}$ materials combine the boons of both 2D layered material properties and quasi-1D material properties. Materials in the $\mathrm{MX}_{3}$ family are fascinating candidates for this purpose due to their reduced in-plane bonding symmetry. ${ }^{2-4}$ These $\mathrm{MX}_{3}$ materials can be classified into three categories (IV-X, V-X, VI-X) based on the metal (M) position in the periodic table. Here, $\mathrm{X}$ is the chalcogen element ( $\mathrm{S}, \mathrm{Se}$, or Te); group IV elements are Ti, $\mathrm{Zr}$, Hf, and Rf; group V elements are V, Nb, Ta, and Db; and group VI elements are $\mathrm{Cr}, \mathrm{Mo}, \mathrm{W}$, and Sg. Among these materials, VI-X materials are reported to be amorphous ${ }^{5}$ and are less reported in the literature. A brief overview of the classification, crystal structures, and properties of widely studied $\mathrm{MX}_{3}$ materials (IV-X, V-X) is provided in Table 1.

In most transition metal trichalcogenides, $\mathrm{MX}_{6}$ trigonal prisms ( $\mathrm{M}$ as the central atom and $\mathrm{X}$ dwelling in the prism triangular base) are stacked so as to obtain $\mathrm{MX}_{3}$ trigonal prismatic chains, and these chains (aligned parallel to the $b$-axis of the unit cell) are covalently bonded through van der Waals forces [Fig. 1a]. ${ }^{6}$ Due to this, these chalcogenides have the tendency to show $1 \mathrm{D}$ characteristics along with anisotropy. Depending on the $\mathrm{X}-\mathrm{X}$ bond lengths in these $\mathrm{MX}_{3}$ crystals, three types of chain arrangement are possible ${ }^{2-4}$ which are outlined below:

$>$ A one-type chain arrangement is present in $\mathrm{ZrSe}_{3}$, with SeSe bond lengths of $0.234 \mathrm{~nm}$ and $\left(\mathrm{Se}_{2}\right)^{2-}$ pairs forming in the trigonal base.

$>$ A two-type chain arrangement is present in $\mathrm{TaSe}_{3}$, with SeSe bond lengths of $0.258 \mathrm{~nm}$ and $0.291 \mathrm{~nm}$ (exceeding the value of $\mathrm{ZrSe}_{3}$ ). $\mathrm{TiS}_{3}$ crystals are an example of this type, with the arrangement: $\mathrm{Ti}^{4+} \mathrm{S}^{2-}\left(\mathrm{S}_{2}\right)^{2-}$.

$\triangleright$ A three-type chain arrangement is present in $\mathrm{NbSe}_{3}$ unit cells, with three Se-Se bonds at the base of the trigonal prismatic chains, short (III), mean (I), and long (II), having bond lengths of $0.237 \mathrm{~nm}, 0.248 \mathrm{~nm}$, and $0.291 \mathrm{~nm}$, respectively. The short bond length $(0.237 \mathrm{~nm})$ is ascribed to $\left(\mathrm{Se}_{2}\right)^{2-}$, which supports the weakening of bonds between two Se atoms and strengthens the bonds between $\mathrm{Nb}$ and Se atoms. These types of arrangement create an intermediate situation with $\mathrm{Nb}^{4+}$ and $\mathrm{Nb}^{5+}$.

\subsection{Crystal structures and properties of IV-X type $\mathbf{M X}_{3}$}

As shown in Table $1, \mathrm{TiX}_{3}, \mathrm{ZrX}_{3}$, and $\mathrm{HfX}_{3}(\mathrm{X}=\mathrm{S}$, Se, or Te) are in the group-IV-X family, falling into the $P 2_{1} / m$ space group, and have been investigated for their remarkable physical and chemical properties. Of these, the crystal and band structures of $\mathrm{TiS}_{3}$ are shown in Fig. 1a-c.,8,9 Different Ti-S bond lengths along the $a(2.65 \AA)$ and $b(2.45 \AA)$ axes consequently result in highly conducting $1 \mathrm{D}$ chains parallel to the $b$-axis and turn out to be the reason for the solid anisotropic properties. ${ }^{2,7}$ In Fig. $1 b,^{8}$ the red dashed lines show the Ti one-dimensional chain, which is also covalently bonded laterally with the $a$ axis, materializing sheets and networks through van der Waals forces. The unit cell of monolayer $\mathrm{TiS}_{3}$ is supposed to be a rectangle, whereas for bulk $\mathrm{TiS}_{3}$, it is a monoclinic crystal with the measured lattice constants of $a=4.973 \AA$ 列 $b=3.433 \AA$ A, $c=$ 8.714 $\AA$, and $\beta=97.74^{\circ}{ }^{2,6}$ The nuclear binding energy of bulk 
Table 1 An overview of the crystal structures and properties of $M X_{3}$

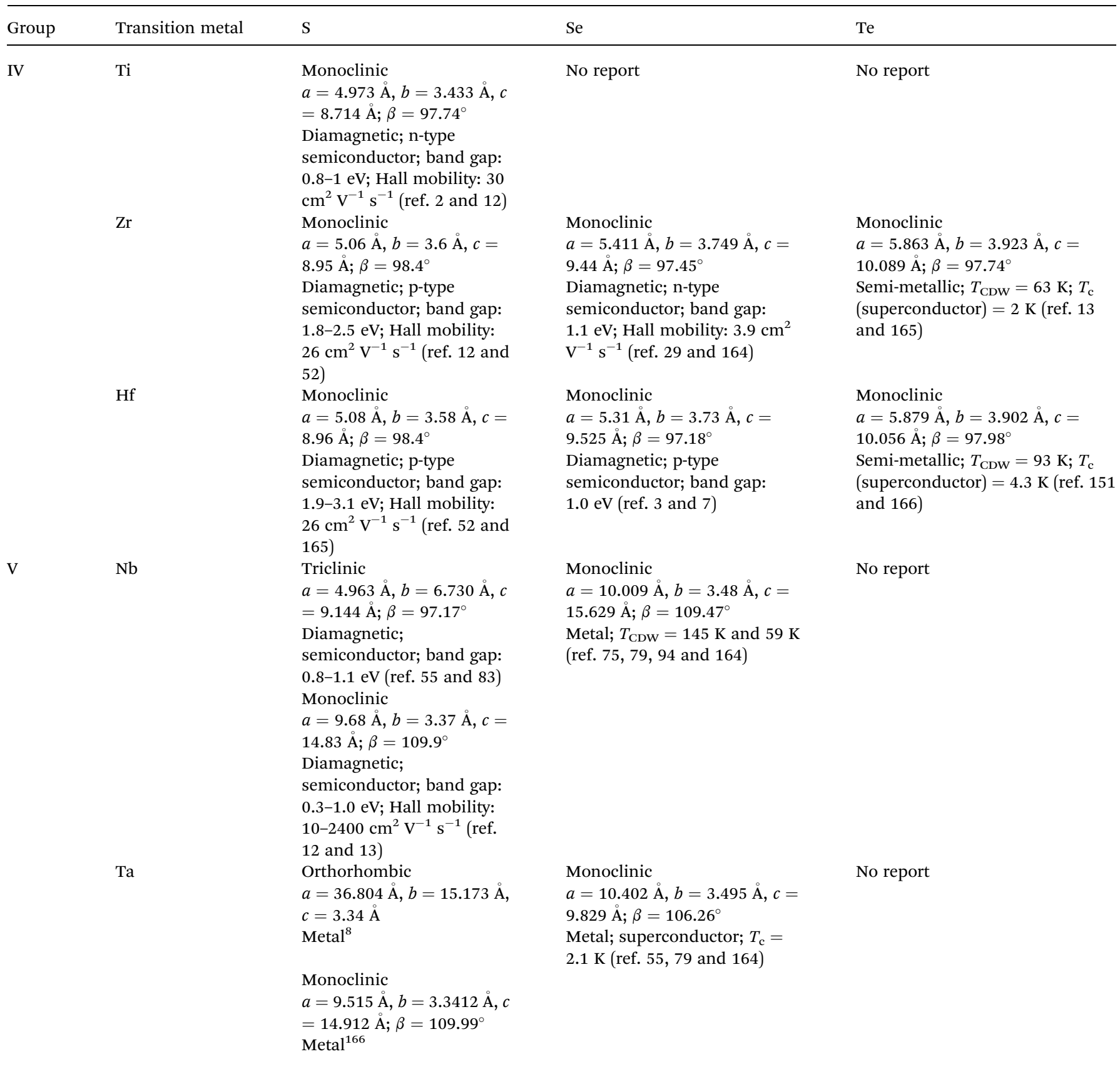

$\mathrm{TiS}_{3}$ is $\sim 0.22$ per unit cell. ${ }^{6,10,11}$ Significantly, there are no reports on $\mathrm{TiSe}_{3}$ and $\mathrm{TiTe}_{3}$.

All members of the $\mathrm{ZrX}_{3}$ and $\mathrm{HfX}_{3}$ family take the form of monoclinic crystals, whose lattice parameters are summarized in Table 1. As discussed, the layered-type crystal structure of $\mathrm{MX}_{3}$ has the metal ion (M) in the centre of a slanted trigonal prism; stacked trigonal faces form separated columns that arrange along the $b$-axis, and the base triangle has a shape with two sides much longer than the third one. ${ }^{\mathbf{1 2 - 1 5}}$ This criterion is satisfied by the formulation $\left(\mathrm{X}^{2-}\right)\left(\mathrm{X}_{2}{ }^{2-}\right)$, with the metal oxidation state being $\mathrm{M}^{4+}\left(\mathrm{d}^{0}\right)$, leading to empty d-block bands. Due to this fact, most $\mathrm{MX}_{3}$ compounds are reported to be semiconducting, with a band gap of $\sim 1-2 \mathrm{eV}$, except $\mathrm{ZrTe}_{3}$ and
$\mathrm{HfTe}_{3}$, which are semi-metallic with superconducting properties at low temperatures. ${ }^{12-15}$ The crystal structure of $\mathrm{ZrTe}_{3}$ is presented in Fig. $4 \mathrm{a}$ and $\mathrm{b} .^{15}$

2.1.1. Optical, electronic, and anisotropic properties of IV$\mathbf{X}$-type $\mathbf{M X}_{3}$. TiS $\mathrm{T}_{3}$ is found to be an n-type semiconductor with an energy band gap of $0.8-1.0 \mathrm{eV}$, and for $N=5$ layers, the band gap is just $24 \mathrm{meV}$ less than that of a single layer $(N=1)$. For that reason, having robust conduction band maximum (CBM) and valance band maximum (VBM) energy states, the energy gap of $\mathrm{TiS}_{3}$ does not change depending on the layer thickness. ${ }^{11}$ First principles calculations on the electronic structures of mono- and few-layered films demonstrate that the properties are quite fixed and do not depend on the number of layers, 
(a)

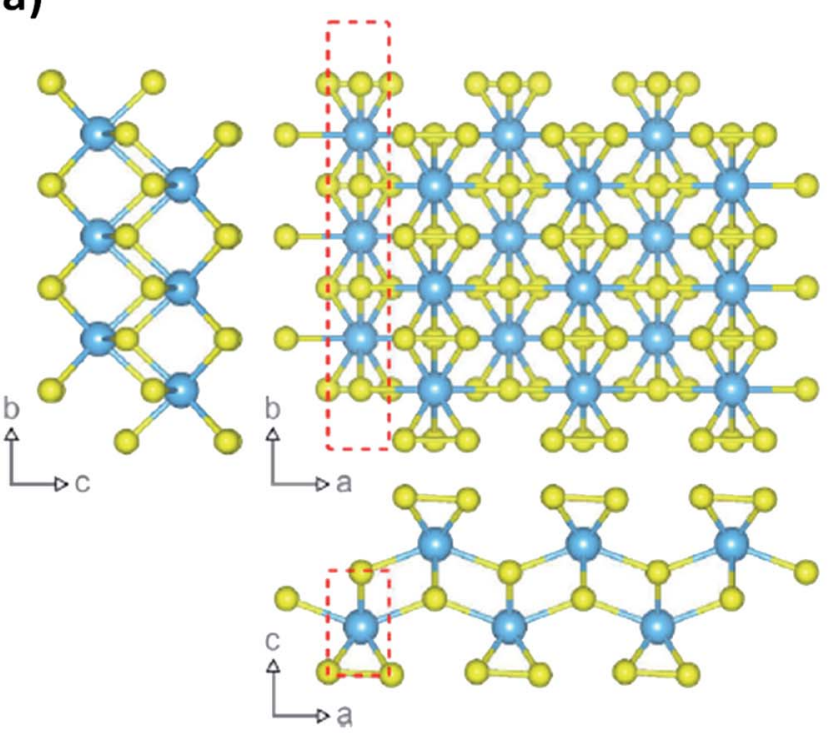

(b)

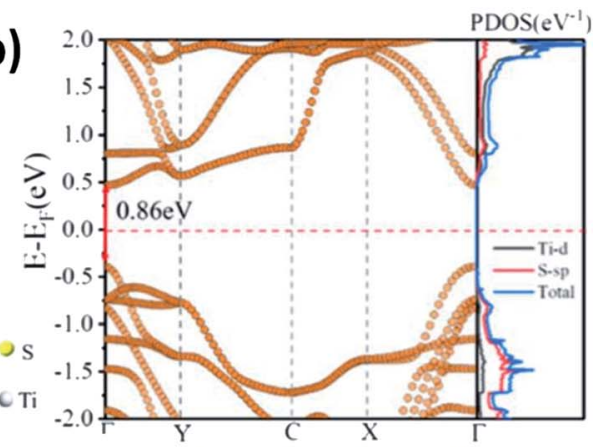

(c)

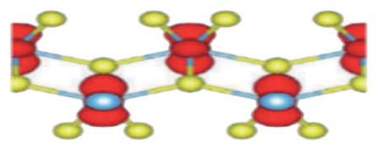

CBM

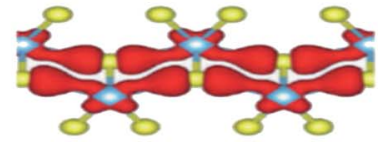

VBM

Fig. 1 (a) The crystal structure of anisotropic $\mathrm{TiS}_{3}$ indicating the bond lengths between titanium and sulfur along the $b$-axis and $a$-axis, with shorter bonds along the $b$-axis; reproduced with permission from ref. 6, copyright: 2015, Springer Nature. (b) The calculated electronic energy band structure along the symmetry directions of the Brillouin zone $(\Gamma-Y-C-X-\Gamma)$ and the projected density of states (PDOS) of the optimized structure of $\mathrm{ML} \mathrm{TiS}_{3}$. The direct band gap $E_{\mathrm{g}}$ of $\mathrm{ML} \mathrm{TiS}_{3}$ is indicated by the red arrows. The Fermi level is set to zero energy (the dashed line); reprinted with permission from ref. 8, copyright: 2019, American Chemical Society. (c) The charge distributions of the VBM and CBM states of monolayer $\mathrm{TiS}_{3}$; republished with permission from ref. 9, permission conveyed through Copyright Clearance Center, Inc.

vertical strain, and piling order. ${ }^{9}$ Gomez et al. measured the electronic band gap and optical band gap to be $1.20 \mathrm{eV}$ (with a rectification factor of $0.08 \mathrm{eV}$ ) and $1.07 \mathrm{eV}$ (with a rectification factor of $0.01 \mathrm{eV}$ ), respectively, hence demonstrating the exciton energy (the difference between the electronic band gap and optical band gap) to be $130 \mathrm{meV} \cdot{ }^{16}$ Biele et al. demonstrated that (a)

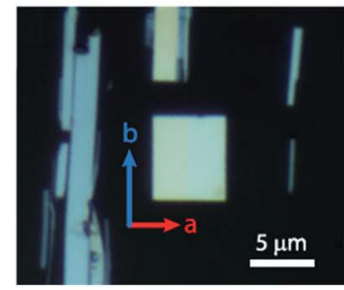

(e)

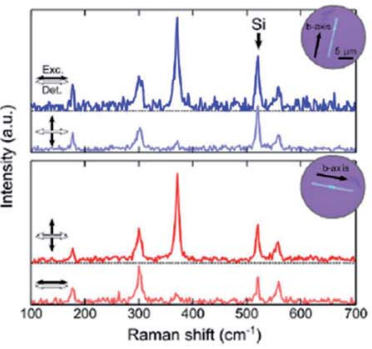

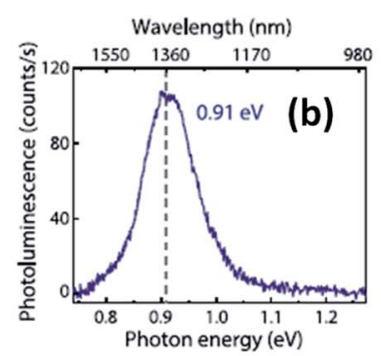
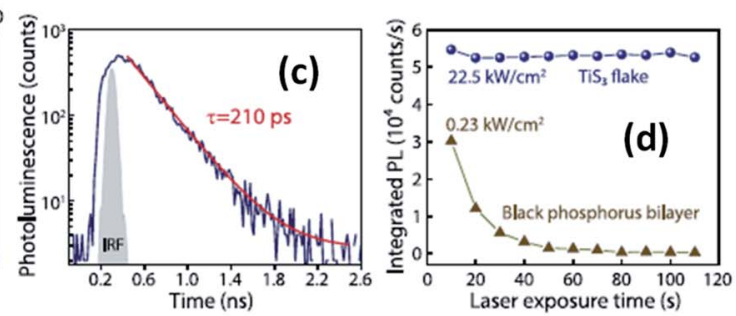

(f)

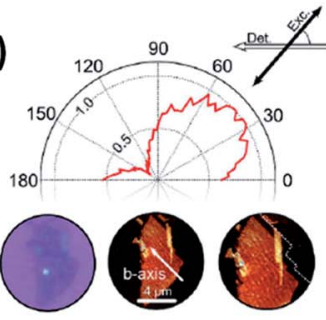

(g)

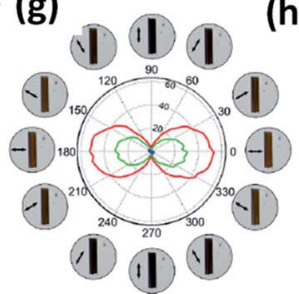

(h)

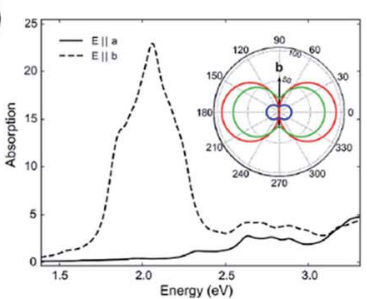

Fig. 2 The optical properties of $\mathrm{TiS}_{3}$ flakes. (a) An optical microscope image showing the $a$ and $b$ axis directions and infrared light emission from $\mathrm{TiS}_{3}$. (b) Photoluminescence and (c) time-resolved photoluminescence spectra and (d) the photostability of a TiS 3 flake compared to black phosphorus. Reproduced with permission from ref. 18, copyright: 2019, IOP Science. (e) Raman spectra of TiS 3 ribbons with horizontal excitation and detection polarization. (f) The intensity of the $370 \mathrm{~cm}^{-1}$ Raman peak of a $3 \mathrm{~nm}$-thick flake (3-4 layers) as a function of the excitation polarization angle (ref. 18). (g) The transmittance of the red, green, and blue channels as a function of the excitation polarization angle. (h) Calculated absorption spectra when the field is aligned parallel to the $b$-axis (dashed line) and the $a$-axis (solid line) with the inset showing the transmittance in the $a-b$ plane for red $(1.9 \mathrm{eV})$, green $(2.4 \mathrm{eV})$, and blue $(2.72 \mathrm{eV})$ excitation energies. Reproduced with permission from ref. 6 ; copyright: 2015, Springer Nature. 
tuning from a direct to an indirect band gap in $\mathrm{TiS}_{3}$ is possible via the application of compressive strain during the course of normal electrical transport. $A b$ initio calculations and optical absorption experiments confirmed a band gap increase of $9 \%$ (from 0.99 to $1.08 \mathrm{eV}$ ) upon tensile stress stimulation. ${ }^{17}$ DFT calculation reports on the strain engineering of $\mathrm{TiS}_{3}$ monolayers demonstrate that the degree of anisotropy in mobility and effective mass can be reformed using tensile strain. ${ }^{11}$

Experimental reports by Khatibi et al. showed that $\mathrm{TiS}_{3}$ emits near infrared (NIR) light centred at about $0.91 \mathrm{eV}(1360 \mathrm{~nm})$ with undeviated polarized anisotropic photoluminescence with a radiation life span of 210 ps (Fig. $2 \mathrm{a}-\mathrm{d}$ ). ${ }^{18}$ The dependence of emission on the excitation power and temperature demonstrated the dominant behaviour of free and bound electronhole pairs over excitonic radiation at room and low temperature, correspondingly. $\mathrm{TiS}_{3}$ is reported to show excellent stable emission compared to other 2D materials, such as black phosphorus, $\mathrm{MoTe}_{2}$, etc. ${ }^{18} \mathrm{TiS}_{3}$ is one of the appealing materials in the $\mathrm{MX}_{3}$ family due to its anisotropic optical properties. By using a force field along either the $a$-axis or $b$-axis and carrying out detection at intermediate angles between $a$ and $b$, the anisotropic optical properties of $2 \mathrm{D} \mathrm{TiS}_{3}$ are investigated. ${ }^{6}$ The anisotropic optical properties survey demonstrated strong emission anisotropy along the $b$-axis $(25 \%$ higher emission intensity than along the $a$-axis). Island et al. inspected the strong in-plane anisotropy of $\mathrm{TiS}_{3}$ through angle-resolved polarization Raman spectroscopy (ARPRS). ${ }^{6}$ Compared to at room temperature, at $25 \mathrm{~K}$, the in-plane conductivity in terms of anisotropy increases 2.09-fold. Fig. 2e and $\mathrm{f}^{6}$ shows Raman studies of $\mathrm{TiS}_{3}$ ribbons under different polarization conditions. Taking the $b$-axis as the favourable growth axis, four distinguishable Raman peaks (excluding one peak due to the silicon substrate) due to $\mathrm{TiS}_{3}$ were observed from an isolated nanoribbon on the $\mathrm{SiO}_{2} / \mathrm{Si}$ substrate. It is reported that the intensities of all the modes can be altered via changing the polarization angle, whereas the peak close to $370 \mathrm{~cm}^{-1}$ is found to be reliant on alignment between the polarization of the excitation laser and the $b$-axis. Furthermore, the anisotropy of the photosensitivity is verified based on the transmission of $\mathrm{TiS}_{3}$ through characteristic angles (Fig. 2h). ${ }^{6}$ The transmission reached a minimum value when the angle between the polarized excitation light source and the elongated side of the flakes was $180^{\circ}$ (parallel to each other) in correspondence with the $b$-axis. The collected optical transmission data confirmed that $\mathrm{TiS}_{3}$ flakes exhibit robust linear dichroism, where the properties along the $b$-axis dominate those of the $a$-axis by as much as 30 -fold, which is commendable for a $2 \mathrm{D}$ material. ${ }^{6}$ Compared to common semiconductors and other 2D TMDCs, $\mathrm{TiS}_{3}$ nanoribbons have a high exciton binding energy, which makes them an excellent applicant for use in optoelectronic devices and various related applications. Iyikanat et al. calculated the band gap of $\mathrm{TiS}_{3}$ using the PBE (Perdew-Burke-Ernzerhof) approximation and HSE06 (Heyd-Scuseria-Ernzerhof functional) correction to be $0.23 \mathrm{eV}$ and $1.05 \mathrm{eV}$, respectively. ${ }^{19}$ The effects of $\mathrm{S}$, Ti, TiS, and double $\mathrm{S}$ vacancies in $\mathrm{TiS}_{3}$ on its optical and electrical properties were also discussed based on DFT calculations by the above group (Fig. 3a-d) ${ }^{19}$ Of the four types of vacancies, except for $\mathrm{S}$ vacancies, the others lose their semiconducting properties and become metallic, resulting in a net magnetic moment. However, the low formation energy in the case of $\mathrm{S}$ vacancies assists in the opening of the energy gap of $\mathrm{TiS}_{3}$ monolayers. The calculated magnetic moments for the $\mathrm{Ti}$, TiS, and double $\mathrm{S}$ vacancies of $\mathrm{TiS}_{3}$ are found to be $0.5,0.8$, and $0.3 \mu_{\mathrm{B}}$ per supercell, respectively. ${ }^{19}$ Kang et al. reported that the microelectronic properties
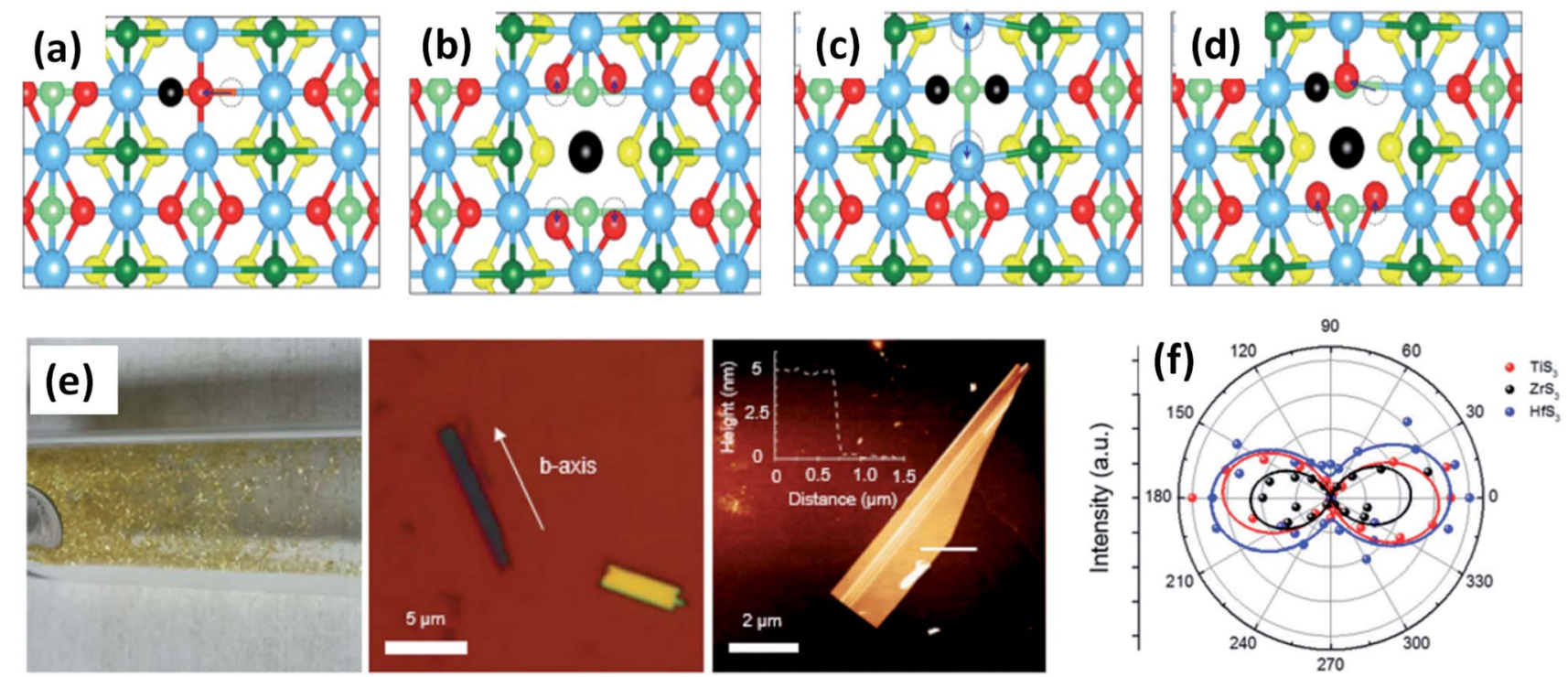

Fig. 3 Top views of relaxed monolayer $\mathrm{TiS}_{3}$ with (a) $\mathrm{S}$, (b) Ti, (c) double $\mathrm{S}$, and (d) TiS vacancies. The black atoms illustrate removed atoms, and the dashed circles show the initial positions of the displaced atoms. Reproduced with permission from ref. 19, copyright: 2015, American Chemical Society. (e) An optical photograph of $\mathrm{HfS}_{3}$ needles grown on the inner walls of a quartz ampoule, an optical image of $\mathrm{ZrS}_{3}$ flakes exfoliated onto $\mathrm{SiO}_{2}$ substrates with the $b$-axis direction shown, and an AFM image of an exfoliated TiS $S_{3}$ flake with a thickness of $\sim 5 \mathrm{~nm}$. (f) A polar plot of mode III at 372 (red), 320 (black), and 321 (blue) $\mathrm{cm}^{-1}$, which corresponds to thin $\mathrm{TiS}_{3}, \mathrm{ZrS}_{3}$, and $\mathrm{HfS}_{3}$ flakes, respectively. Republished with permission from ref. 24, permission conveyed through Copyright Clearance Center, Inc. 
of $\mathrm{TiS}_{3}$ nanoribbons strongly depend on the side, whether it is $a$ or $b .{ }^{20} a-\mathrm{TiS}_{3}$ nanoribbons are reported to have the properties of a metal, having a width-dependent band gap, whereas $b$-TiS is a semiconductor with a direct energy gap, which can be regulated by strain, is nearly self-governing, and does not hinge on the ribbon width. ${ }^{20}$

$\mathrm{ZrS}_{3}$ is reported to be a p-type semiconductor, with resistivity of $15 \Omega \mathrm{cm}$ at room temperature, a direct optical energy gap of 1.8-2.5 eV, and two indirect optical energy gaps of $2.055 \mathrm{eV}$ (when the field is along the $b$-axis) and $2.058 \mathrm{eV}$ (when the field is normal to the $b$-axis) at $4.2 \mathrm{~K}^{4}{ }^{4}$ Angle-resolved photoemission and optical measurements of $\mathrm{ZrX}_{3}(\mathrm{X}=\mathrm{S}$ or $\mathrm{Se})$ and $\mathrm{HfSe}_{3}$ predict that, due to spin-orbit interactions, there is splitting of the highest occupied band into two parallel bands. The split energy rises from $\mathrm{S}$ to $\mathrm{Se}$ in the above compound, and the absorption spectra are found to be highly anisotropic considering the phonon modes and electronic transitions. ${ }^{21}$ Schairer et al. evaluated the optical gap energies of $\mathrm{ZrS}_{3}$ and $\mathrm{HfS}_{3}$ to be 2.8 and $3.1 \mathrm{eV} .^{12}$ In another report, Jandl et al. confirmed the presence of two polytypes in accordance with the recombination of phonon replicas of excitons localized in band tails. ${ }^{22}$ Pant et al. reported the indirect band gap of $\mathrm{ZrS}_{3}$ to be $1.88 \mathrm{eV}$, and angle-resolved photoluminescence (PL) spectroscopy studies of $\mathrm{ZrS}_{3}$ nanosheets demonstrated that they are highly anisotropic, which is manifested by the large PL intensity variation with polarization direction. ${ }^{23}$ Raman spectroscopy and angleresolved studies are fast and non-destructive optical methods to probe the anisotropic nature of $\mathrm{MX}_{3}$. In this regard, Kong et al. collected Raman data from $\mathrm{MX}_{3}$ flakes $\left(\mathrm{TiS}_{3}, \mathrm{ZrS}_{3}\right.$, and $\mathrm{HfS}_{3}$ ) with the longer edge (the anisotropic $b$-axis) aligned parallel to both the polarization direction of the laser and the polarization exposure path of the Raman spectrometer. ${ }^{24}$ To the contrary, for $\mathrm{TiNbS}_{3}$ alloy trichalcogenides, it is not possible to determine the anisotropic direction due to the loss of anisotropy and common defects present in the random distribution of quasi-1D $\mathrm{MX}_{6}$ chains. ${ }^{24}$ Wang et al. verified the anisotropic effects in $\mathrm{ZrS}_{3}$ via angle-resolved absorption and angle-resolved Raman spectroscopy, and angle-resolved photocurrent studies, which gave a vibrant impression that all the properties were boosted along the $b$-axis. Angle-resolved spectroscopy studies revealed dichroic ratios of 1.73 and 1.14 for the $\mathrm{ZrS}_{3}$ nanoribbons when excited by different laser source wavelengths, i.e., $450 \mathrm{~nm}$ and $532 \mathrm{~nm}$, respectively, considering the photocurrent density. ${ }^{25}$ The change in dichroic ratio is owing to the variation of the offset angle from the $b$-axis. Jin et al. showed the widening and trivial shifting of peaks in the Raman spectra due to the phonon confinement effect in the cases of $\mathrm{ZrS}_{3}$ and $\mathrm{HfS}_{3}$ nanobelts. ${ }^{26}$ Likewise, $\mathrm{TiS}_{3}$ Raman spectra collected by Pawbake et al. showed the shifting of the peaks towards lower wavenumbers upon an increase in temperature ( $88 \mathrm{~K}$ to $570 \mathrm{~K}$ ), which is attributed to thermal expansion of the lattice and anharmonic vibration. ${ }^{27}$ Apart from temperature, pressuredependent Raman spectroscopy studies were done by $\mathrm{Wu}$ et al., which describe the unorthodox negative pressure dependence of the $\mathrm{A}_{\mathrm{g}}^{\mathrm{S}-\mathrm{S}} \mathrm{S}-\mathrm{S}$ molecular mode in contrast to the expected stiffening of other peaks. Numerous modes of $\mathrm{TiS}_{3}$ are reported to be doubly degenerate at ambient pressure, whereas at high pressure, this effect vanishes. ${ }^{28}$

Patel et al. calculated the direct and indirect energy gaps of $\mathrm{ZrSe}_{3}$ to be around $1.47 \mathrm{eV}$ and $1.1 \mathrm{eV}$, respectively. They also found that by means of an escalation in temperature in the vicinity of 303-403 $\mathrm{K}$, the anisotropy and resistivity could be decreased and increased, respectively. ${ }^{29}$ In agreement with quasi particle self-energy correction, Zhou et al. found the indirect band gap of $\mathrm{ZrSe}_{3}$ to be $1.63 \mathrm{eV} \cdot{ }^{30}$ Felser et al. established a dependent relationship between the density of states and the shape of the Fermi surface in Te-Te interprism interactions in $\mathrm{ZrTe}_{3} .{ }^{14}$ Zeng et al. showed that growing strain in $\mathrm{HfS}_{3}$ resulted in a switch from an indirect to a direct band gap of $2.2 \mathrm{eV}$, whereas Tao and his group calculated the indirect and direct optical energy gaps of $\mathrm{HfS}_{3}$ nanobelts to be 1.73 and $2.19 \mathrm{eV}$, respectively. ${ }^{\mathbf{3 1 , 3 2}}$ As shown by photoluminescence (PL) studies, the nanobelts displayed strong emission at 483, 540, and $600 \mathrm{~nm}$ in response to excitation at $400 \mathrm{~nm} .{ }^{32}$ Likewise, Zhao et al. calculated the band gaps for $\mathrm{Zrs}_{3}, \mathrm{Zrse}_{3}, \mathrm{Hfs}_{3}$, and $\mathrm{Hfse}_{3}$ to be $1.13,0.23,1.08$, and $0.05 \mathrm{eV}$, respectively. According to their study, the p state of the chalcogen (governing the VBM) and the $\mathrm{d}$ state of the transition metal (governing the CBM) combined to form the band gap of the $\mathrm{MX}_{3}$ monolayers. ${ }^{33}$

Trisulphides, for example $\mathrm{ZrS}_{3}$ - and $\mathrm{HfS}_{3}$-based structures, are reported to exhibit two dozen normal modes at the centre of the Brillouin zone and belong to the $C_{2 \mathrm{~h}}$ space group..$^{26,34,35}$ Each mode is interconnected with a clutch of atomic vibrations represented by $A_{g}, B_{g}, A_{u}$, and $B_{u}$. Among these, the $A_{u}$ and $B_{g}$ vibrations are parallel to the $\mathrm{MX}_{6}$ trigonal prism chains, and the $\mathrm{A}_{\mathrm{g}}$ and $\mathrm{A}_{\mathrm{u}}$ bands are perpendicular to the chains. Gleason et al. investigated the pressure-induced phases of $\mathrm{ZrTe}_{3}$ and also analysed the temperature-dependent Raman spectra. ${ }^{36}$ It is reported that some specific phonon modes endure a dramatic linewidth reduction near the charge density wave temperature ( $\left.T_{\mathrm{CDW}}\right)$, indicating the strong coupling of phonons with electronic degrees of freedom concomitant with the CDW (Fig. 4). Typical Raman spectra from a $\mathrm{ZrTe}_{3}$ crystal are shown in Fig. 4c at $295 \mathrm{~K}$ and $6 \mathrm{~K}$ with normal mode displacement patterns. The lower-energy modes $\left(\omega_{1}-\omega_{3}\right)$ and higher-energy modes $\left(\omega_{4}-\omega_{6}\right)$ are allocated primarily to vibrations of the trigonal prismatic rods, inwardly and outwardly, respectively. Changes in the widths of the Raman bands at different temperatures (Fig. 4d and e $)^{36}$ are observed, with the large reductions in the widths of $\omega_{4}$ and $\omega_{5}$ demonstrating strong coupling between these bands. Similarly, the downfall of the long-range-order (LRO) of the rods is attributed to the suppression of phonon bands allied to internal vibrations of the $\mathrm{ZrTe}_{3}$ prismatic rods at pressures above 10 kbar (Fig. $4 \mathrm{f}$ and $\mathrm{g}$ ). ${ }^{36}$

2.1.2. Electrical and magnetic properties of IV-X-type $\mathbf{M X}_{\mathbf{3}}$. The electronic superstructure and projected density of states (PDOS) along the symmetry direction of the Brillouin zone of the augmented assembly of monolayer $\mathrm{TiS}_{3}$ are shown in Fig. $1 \mathrm{~b} .{ }^{8}$ Fig. $1 \mathrm{c}$ depicts the distribution of charge in the VBM and CBM of monolayer $\mathrm{TiS}_{3} .{ }^{9}$ These details give a clear-cut idea about the high carrier mobility of $2 \mathrm{D} \mathrm{TiS}_{3}$ monolayers. The electron mobilities of $\mathrm{TiS}_{3}$ are reported to be high and anisotropic, with electron mobility of $13.87 \times 10^{3} \mathrm{~cm}^{2} \mathrm{~V}^{-1} \mathrm{~s}^{-1}$ along 
the $b$-axis, which is about 14 times greater than that along the $a$ axis $\left(1.01 \times 10^{3} \mathrm{~cm}^{2} \mathrm{~V}^{-1} \mathrm{~s}^{-1}\right)$, while the hole mobility along the $a$-axis is $1.21 \times 10^{3} \mathrm{~cm}^{2} \mathrm{~V}^{-1} \mathrm{~s}^{-1}$, about eight times higher than that along the $b$-axis $\left(0.15 \times 10^{3} \mathrm{~cm}^{2} \mathrm{~V}^{-1} \mathrm{~s}^{-1}\right) .{ }^{10}$ DFT calculation reports on the strain engineering of $\mathrm{TiS}_{3}$ monolayers demonstrated that the extent of the anisotropy of both mobility and effective mass changed as a result of the influence of tensile strain. Strain engineering leads to an order-of-magnitude increase in the mobility of acoustic phonons at $300 \mathrm{~K}(100 \mathrm{~K})$, i.e., from $1.71 \times 10^{4}\left(5.13 \times 10^{4}\right) \mathrm{cm}^{2} \mathrm{~V}^{-1} \mathrm{~s}^{-1}$ to $5.53 \times 10^{5}(1.66$ $\left.\times 10^{6}\right) \mathrm{cm}^{2} \mathrm{~V}^{-1} \mathrm{~s}^{-1} \cdot{ }^{11}$ Investigations into the temperature dependence of resistance along the chains ( $b$-axis) and across the chains ( $a$-axis) of $\mathrm{TiS}_{3}$ are reported to show non-linear conductivity. The observed non-linear conduction in $\mathrm{TiS}_{3}$ brings to mind quasi-1D conducting behaviour with a sliding charge density wave. ${ }^{37}$

In quasi-1D conductors, the condensations of electrons into CDWs arises due to Fermi surface instability, and they form a deformable medium that affects their overall static and dynamic properties, giving rise to metallic stability and hysteresis. Low-dimensional materials, including anisotropic $2 \mathrm{D} \mathrm{MX}_{3}$ layered structures, from time to time lose their LRO and orthodox symmetry, resulting in Fermi surface instability and forming a presumed charge density wave (CDW). ${ }^{38,39} \mathrm{ZrTe}_{3}$ is one attention-grabbing material in the type IV-X $\mathrm{MX}_{3}$ family, which is reported to have anisotropic nature, with a CDW at 63 $\mathrm{K}$ and superconductivity at $2 \mathrm{~K}^{\mathbf{. 4 , 1 5 , 4 0 - 4 2}}$ Other reports on $\mathrm{ZrTe}_{3}$ revealed a CDW transition at $63-70 \mathrm{~K}$ due to resistivity inconsistency along the $a$-axis, but not along the conventional anisotropic $b$-axis. ${ }^{41,43}$ Canadell et al. predicted that $\mathrm{ZrTe}_{3}$ is a type-B structure with shorter $\mathrm{X}-\mathrm{X}$ contacts between adjacent $\mathrm{MX}_{3}$ units. The type-B structure of $\mathrm{ZrTe}_{3}$ plays a crucial role in determining the semi-metallic nature of the $\mathrm{ZrTe}_{3}$ chains. ${ }^{13} \mathrm{Zhu}$ et al. reported a comparison of the electrical and superconducting properties of $\mathrm{ZrTe}_{3}$ single crystals prepared at low $\left(735{ }^{\circ} \mathrm{C}\right)$ and high $\left(950{ }^{\circ} \mathrm{C}\right)$ temperatures. ${ }^{15}$ From resistive, colorimetric, and magnetic studies, bulk superconductivity is perceived in the HT-ZrTe ${ }_{3}$ crystals with the help of doping after a certain temperature, i.e., $4 \mathrm{~K}$, but not in the $\mathrm{LT}-\mathrm{ZrTe}_{3}$ crystals. The difference in electrical properties is attributed to the suppression of CDWs through growth-induced structural disorder at high temperature. ${ }^{15}$ Polarized Raman measurements and first principles calculations demonstrated that precise structural vibrational arrangements from longitudinal distortions of the Te(II)-Te(III) chains had a strong association with the conduction of electrons, leading to the formation of CDWs in $\mathrm{ZrTe}_{3} \cdot{ }^{44-46}$ Pressure-dependent electrical properties investigations revealed that the CDW transition temperature $\left(T_{\mathrm{CDW}}\right)$ of $\mathrm{ZrTe}_{3}$ was initially amplified, then diminished at $2 \mathrm{GPa}$, and quickly vanished at $5 \mathrm{GPa}$, but superconductivity was shown at a pressure level of up to $11 \mathrm{GPa} .{ }^{47}$ However, Hoesch et al. performed low-temperature and high-pressure single crystal X-ray diffraction studies along with $a b$ initio DFT studies to show that the reported abrupt demise of the CDW phase is due to instability in the Fermi surface above $5 \mathrm{GPa}^{48} \mathrm{Zhu}$ et al. reported the presence of different bands, including bands with flat and dispersive profiles, along with the amalgamation of chalcogen (high mobility) and metal (low mobility) derived bands in the Fermi surface of $\mathrm{ZrTe}_{3}$. Due to the suppression of long-range CDW order, superconductivity materialises in Se-
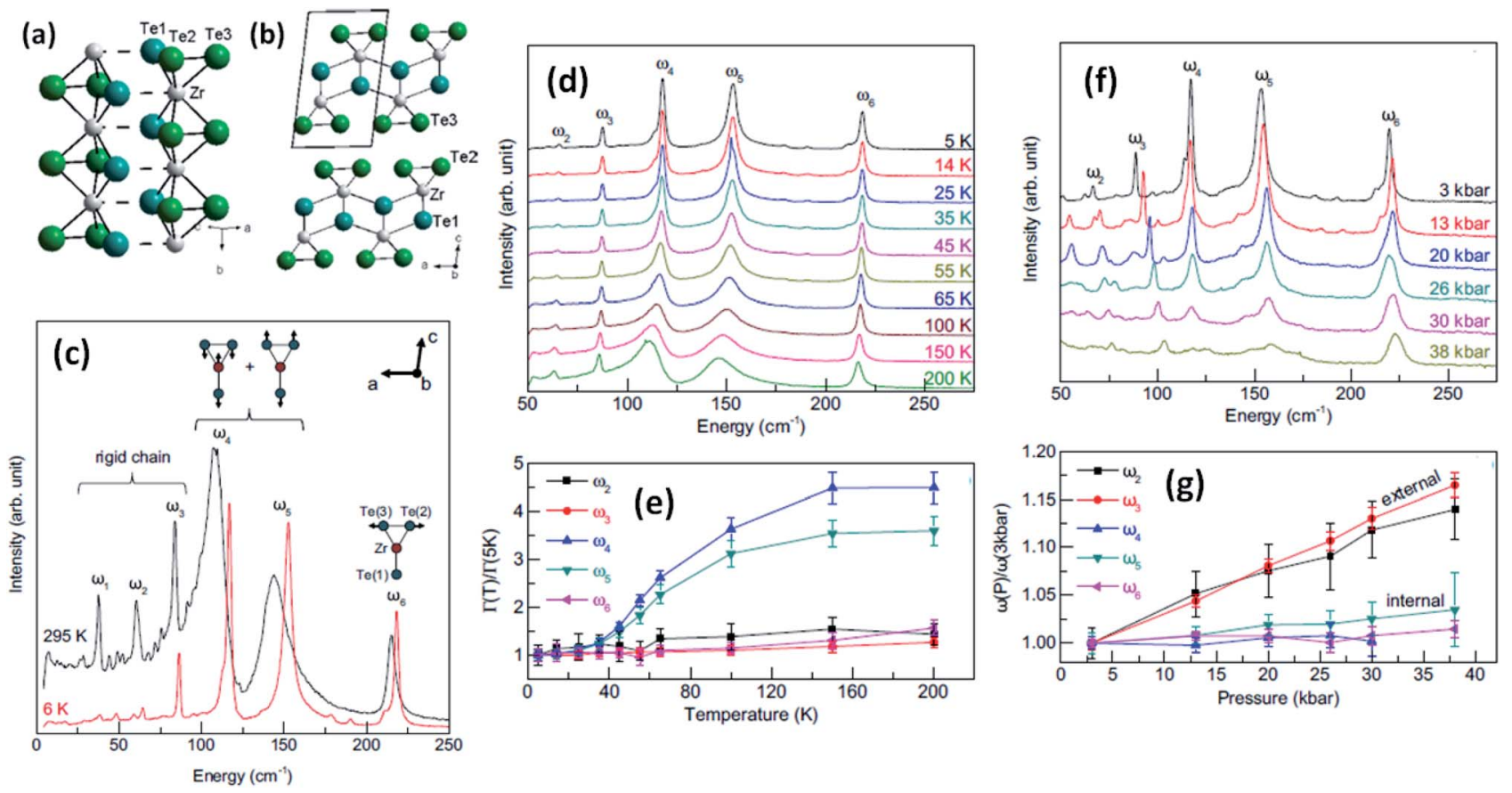

Fig. 4 The crystal structure of $\mathrm{ZrTe}_{3}$ : (a) quasi-one-dimensional trigonal prism packing along the $b$ axis and (b) a quasi-two-dimensional ZrTe 3 layer along the $a-c$ plane; reprinted with permission from ref. 15, copyright: 2013, American Physical Society. (c) Raman spectra of ZrTe 3 at $6 \mathrm{~K}$ and 295 K. ( $d$ and e) Temperature-dependent Raman spectra at ambient pressure and ( $\mathrm{f}$ and g) pressure-dependent Raman spectra at $T=3 \mathrm{~K}$. Reprinted with permission from ref. 36, copyright; 2013, American Physical Society. 
doped $\mathrm{ZrTe}_{3} \cdot{ }^{49}$ The superconductivity critical temperature $\left(T_{\mathrm{c}}\right)$ increased up to $4 \mathrm{~K}$, but after that, additional Se doping caused a reduction in $T_{\mathrm{c}}$ and filamentary superconductivity in $\mathrm{ZrTe}_{3-x} \mathrm{Se}_{x}(0 \leq x \leq 0.1) .{ }^{49}$ Polycrystalline $\mathrm{ZrTe}_{3}$ is testified to show a superconducting transition temperature of $5.2 \mathrm{~K}$ and a $T_{\mathrm{CDW}}$ value of $\sim 63 \mathrm{~K}$, both co-existing at ambient pressure. ${ }^{50}$ The intercalation of $\mathrm{Ag}$ and $\mathrm{Cu}$ into $\mathrm{ZrTe}_{3}$ favoured an escalation in electrical conductivity and resulted in CDW anomalies, which is confirmed from DC magnetisation results, whereas it had no role on $T_{\mathrm{CDW}}$ and $T_{\mathrm{c} \cdot}{ }^{50} \mathrm{Li}$ et al. reported CDW formation at $T_{\mathrm{CDW}}=93 \mathrm{~K}$ with typical anisotropy. ${ }^{51}$ Conductivity measurements parallel to the $a$-axis $\left(\rho_{a}\right)$ and $b$-axis $\left(\rho_{b}\right)$ of a $\mathrm{HfTe}_{3}$ crystal gave a direct indication of the disorder-related superconductor fluctuations in $\rho_{b}$ at $4.3 \mathrm{~K}$. Also, a superconducting phase with $T_{\mathrm{c}}=1.7 \mathrm{~K}$ co-exists with a lower $T_{\mathrm{CDW}}$ value of $80 \mathrm{~K}$ in a polycrystalline sample, whose generation could be attributed to enriched disorder scattering or unintended carrier doping. ${ }^{51}$ A combined experimental and computational study by $\mathrm{Hu}$ et al. explored a new concept, which emphasizes that phonon-electron coupling helps in the construction of CDWs in $\mathrm{ZrTe}_{3}$. However, their study depicted that the breaching of fractional electronic gaps in the CDW state depends upon the phonon-electron momentum and coupling. ${ }^{44}$ The resistivity and Hall mobility at room temperature of $\mathrm{ZrSe}_{3}$ were calculated by Ikari et al. to be about $9 \times 10^{2}$ $\Omega \mathrm{cm}$ and $0.45 \mathrm{~cm}^{2} \mathrm{~V}^{-1} \mathrm{~s}^{-1}$, respectively. ${ }^{52}$

Lai et al. reported a comparative investigation into the magnetic properties and tensile strain response of $\mathrm{N}-a(b)-\mathrm{TiS}_{3}$ nanoribbons, where $a-\mathrm{TiS}_{3}$ and $b$-TiS ${ }_{3}$ are nanoribbons reviewed along either the $a$ - or $b$-axis and $N$ indicates the number of $\mathrm{Ti}$ atoms in the monoclinic cell of the ribbons. ${ }^{53}$ It was found that the magnetic ground state was a ferromagnetic (FM) metal when $N$ was equal to an odd number, whereas it behaved like an antiferromagnetic (AFM) metal when $N$ was equal to an even number for $\mathrm{N}$ - $a$-TiS $\mathrm{T}_{3}$ nanoribbons. Tensile strain (6\%) could be used to tune 9- $a(b)-\mathrm{TiS}_{3}$ nanoribbons from a FM metal to a half metal. Similarly, tensile strain (4\%) also could cause an AFM to FM transition in 10- $a$ - TiS $_{3}$ nanoribbons..$^{53}$

\subsection{Crystal structures and properties of $V-X$-type $M_{3}$}

Apart from IV-X-type $\mathrm{MX}_{3}, \mathrm{~V}$-X-type $\mathrm{MX}_{3}$ has its own presence in the family of metal trichalcogenides with extraordinary physical, chemical, and electrical properties. $\mathrm{NbX}_{3}$ and $\mathrm{TaX}_{3}$ materials with $\mathrm{X}=\mathrm{S}$, Se, or Te belong to the $\mathrm{V}-\mathrm{X}$ family and are strongly anisotropic materials consisting of conducting chains weakly attached by van der Waals forces (Table 1). ${ }^{54,55}$ Among these $\mathrm{MX}_{3}$ materials, $\mathrm{NbS}_{3}$ is reported to have six types of polymorph, which are summarized in Table $2 . \mathrm{NbS}_{3-\mathrm{I}}$ has a monoclinic crystal structure, which was first proposed in 1960 and experimentally verified via single-crystal X-ray diffraction studies in $1978 . .^{56,57} \mathrm{NbS}_{3-\mathrm{I}}$ is reported to be a semiconducting material with a band gap of $\sim 0.66-1.0 \mathrm{eV}$, and its important feature is the bond-pairing between two $\mathrm{Nb}$ atoms along the $\mathrm{NbS}_{3}$ primary chain axis, with a bond length of $\sim 3 \AA$ and creating a $3.7 \AA$ space. ${ }^{58-62}$ The $\mathrm{NbS}_{3 \text {-II }}$ polymorph was first acknowledged in 1978 following electron diffraction experiments, with weak pair satellite diffraction streaks that resolve into rows of spots upon modifications in temperature. ${ }^{63,64}$ It is proposed that $\mathrm{NbS}_{3-\mathrm{II}}$ is an elevated structure of $\mathrm{NbS}_{3-\mathrm{I}}$ with different types of chain accretion, and the rows of spots arise at random positions with analogous separation to $\mathrm{NbS}_{3-\mathrm{I}}$ (Table 2). $\mathrm{NbS}_{3-\mathrm{II}}$ is testified to have three CDWs at $150 \mathrm{~K}, 330-370 \mathrm{~K}$, and 620-650 K. ${ }^{61}$ In 1982, Kikkawa and co-workers obtained monoclinic $\mathrm{NbS}_{3-\mathrm{HP}}$ via the extraordinary high-pressure modification of $\mathrm{NbS}_{3}$ synthesized at $700{ }^{\circ} \mathrm{C}$ with $2 \mathrm{GPa}$ pressure. ${ }^{\mathbf{6 5 , 6 6}}$ Zettl et al. produced $\mathrm{NbS}_{3-\mathrm{III}}$ in 1982 , which was distinctively different from the I and II phases. ${ }^{67}$ In $\mathrm{NbS}_{3-\mathrm{III}}$, (001) reflections from the XRD data showed a similar $c$-axis as that reported for $\mathrm{NbS}_{3-\mathrm{I}}$, but the monoclinic angle was increased to $98^{\circ}-99^{\circ}$ and $T_{\text {CDW }}$ was $\sim 155 \mathrm{~K}$. Zybtsev confirmed the low and high ohmic nature of $\mathrm{NbS}_{3}$, with CDW transitions at $150 \mathrm{~K}$ (both low and high ohmic) and $360 \mathrm{~K}$ (low ohmic). ${ }^{66}$ The low ohmic and high ohmic $\mathrm{NbS}_{3}$ was assigned as $\mathrm{NbS}_{3-\mathrm{II}}$ and $\mathrm{NbS}_{3-\mathrm{III}}$, respectively, with $\mathrm{NbS}_{3-\mathrm{III}}$ designated as a sub-phase of $\mathrm{NbS}_{3-\mathrm{II}}$. For the first time, Bloodgood et al. reported $\mathrm{NbS}_{3-\mathrm{IV}}$ and $\mathrm{NbS}_{3-\mathrm{v}}$ polymorphs of the $\mathrm{NbS}_{3}$ monoclinic crystal structure. ${ }^{55}$ The $\mathrm{NbS}_{3-\mathrm{IV}}$ structure is constructed from $\mathrm{NbS}_{6}$ trigonal prismatic chains with a bond length between $\mathrm{Nb}$ atoms of $3.0448 \AA$, and the chain axis is along the $a$-axis instead of the $b$-axis like other conventional $\mathrm{MX}_{3}$ materials. For $\mathrm{NbS}_{3-\mathrm{V}}$, the $\mathrm{Nb}-\mathrm{Nb}$ bond length is $3.358 \AA$. The bonds in $\mathrm{NbS}_{3-\mathrm{IV}}$ and $\mathrm{NbS}_{3-\mathrm{V}}$ are along the chains, with an $\mathrm{AB}$ and $\mathrm{ABCDE}$ recapping categorization of chain bilayers, analogous to $\mathrm{NbS}_{3-\mathrm{I}}$. The crystal structures of $\mathrm{NbS}_{3-\mathrm{I}}, \mathrm{NbS}_{3-\mathrm{IV}}$, and $\mathrm{NbS}_{3-\mathrm{v}}$ are given in Fig. 5, with a complete overview of unit cells and layers from different perspectives. In the case of $\mathrm{NbS}_{3}$ -

Table 2 Summarized data of the crystal structures, synthesis conditions, and $\mathrm{Nb}-\mathrm{Nb}$ bond lengths of different phases of $\mathrm{NbS}_{3} \mathrm{Crystals}$

\begin{tabular}{|c|c|c|c|c|}
\hline Material & Crystal structure $a, b, \mathrm{c}(\AA) ; \alpha, \beta, \gamma\left(\left(^{\circ}\right)\right.$ & Synthesis conditions & $\mathrm{Nb}-\mathrm{Nb}(\AA)$ & Ref. no. \\
\hline $\mathrm{NbS}_{3-\mathrm{I}}$ & $4.963,6.730,9.144 ; 90,97.17,90$ & $\mathrm{NbS}_{2} \mathrm{Cl}_{2} ; 588{ }^{\circ} \mathrm{C}$ (source) $/ 569^{\circ} \mathrm{C}($ sink $) ; 48 \mathrm{~h}$; slow cooling & $\begin{array}{l}3.045 \\
3.702\end{array}$ & 57 \\
\hline $\mathrm{NbS}_{3-\mathrm{HP}}$ & $9.68,3.37,14.83 ; 90,109.9,90$ & $\mathrm{Nb}+\mathrm{S} ; 700{ }^{\circ} \mathrm{C}$ at $2 \mathrm{GPa} ; 0.5 \mathrm{~h}$ & 3.370 & 131 \\
\hline $\mathrm{NbS}_{3-\mathrm{II}}$ & $9.9,3.4,18.3 ; 90,97,90$ & $\mathrm{Nb}+\mathrm{S} ; 600{ }^{\circ} \mathrm{C}($ source $) / 580{ }^{\circ} \mathrm{C} ; 15$ days & - & 61 and 99 \\
\hline $\mathrm{NbS}_{3-\mathrm{II}}$ & $9.1-9.6,18.7-19.9,3.4 ; 90,97-98,90$ & $\mathrm{Nb}+\mathrm{S} ; 500{ }^{\circ} \mathrm{C}$ & - & 60 \\
\hline $\mathrm{NbS}_{3-\mathrm{III}}$ & $\sim 5,-, \sim 9 ; 90,98-99,90$ & $\mathrm{Nb}_{2}+\mathrm{S} ; 550{ }^{\circ} \mathrm{C} ; 21$ days; $400{ }^{\circ} \mathrm{C}, 48 \mathrm{~h}(48 \mathrm{~h}$, air quenching $)$ & - & 67 \\
\hline $\mathrm{NbS}_{3-\mathrm{IV}}$ & $\begin{array}{l}\text { 6.7515(5), } 4.9736(4), 18.1315(13) ; 90 \\
90.116(2), 90\end{array}$ & $\mathrm{Nb}+\mathrm{S}, \mathrm{I}_{2}$ transport, $70{ }^{\circ} \mathrm{C}($ source $) / 570{ }^{\circ} \mathrm{C}(\operatorname{sink}), 10$ days & $\begin{array}{l}3.0448(8) \\
3.7087(8)\end{array}$ & 55 \\
\hline $\mathrm{NbS}_{3-\mathrm{V}}$ & $\begin{array}{l}4.950(5), 3.358(4), 9.079(10) ; 90 \\
97.35(2), 90\end{array}$ & $\begin{array}{l}\mathrm{Nb}+\mathrm{S}, 10 \% \mathrm{~S}, \mathrm{I}_{2} \text { transport, } 670{ }^{\circ} \mathrm{C}(\text { source }) / 570{ }^{\circ} \mathrm{C}(\text { sink }), \\
10 \text { days }\end{array}$ & $3.358(4)$ & 55 \\
\hline
\end{tabular}




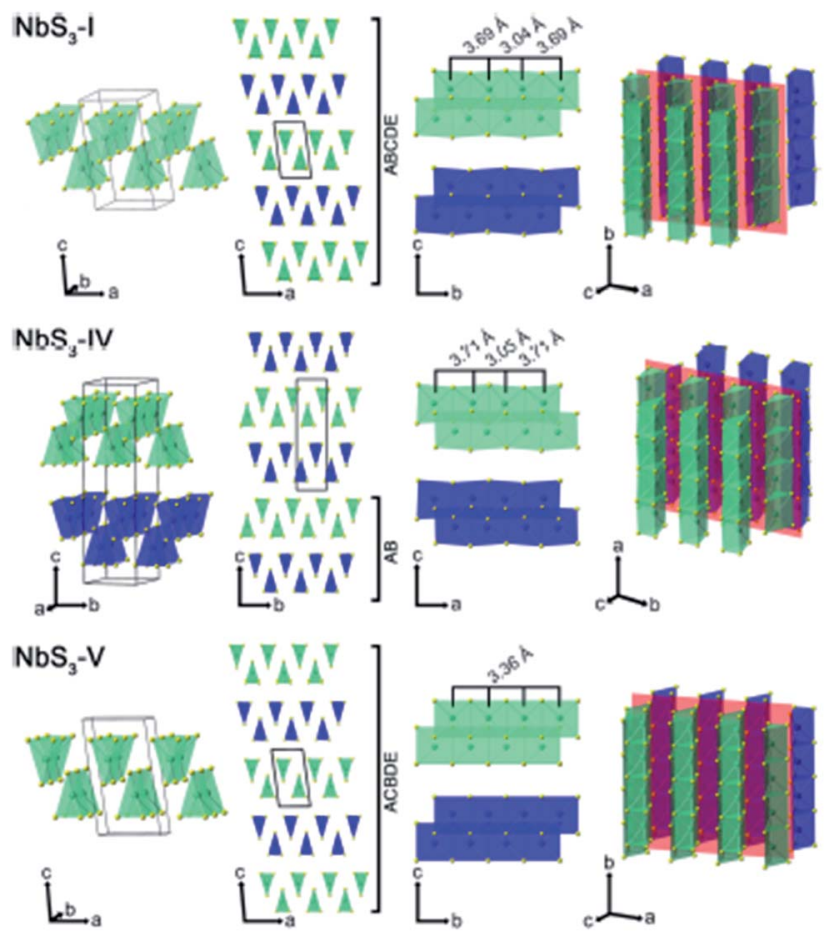

Fig. 5 The crystal structure of $\mathrm{NbS}_{3-1}, \mathrm{NbS}_{3-\mathrm{IV}}$, and $\mathrm{NbS}_{3-\mathrm{v}}$ : unit cells, chain cross-sections, layers, and perspective views. Reproduced with permission from ref. 55, copyright: 2017, Application Infrastructure Provider

Iv, there are up to twice as many chains per unit cell compared to $\mathrm{NbS}_{3-\mathrm{I}}$, since the $c$-axis is doubled, and it displays the properties of a semiconductor. ${ }^{55}$
In 1975, single-crystal $\mathrm{NbSe}_{3}$ was prepared by Meerschaut et al., and single-crystal XRD studies established the monoclinic structure, consisting of inestimable selenium trigonal prismatic chains loaded on top of each other with shared triangular faces. ${ }^{68-70}$ Similar to other $\mathrm{MX}_{3}$-based $2 \mathrm{D}$ materials, the structural arrangement with strong chemical bonding anisotropy makes $\mathrm{NbSe}_{3}$ crystals a tempting material with novel physical and chemical properties. The Se-Se bond lengths are testified to be $2.37 \AA$ and $2.49 \AA$, respectively, with CDWs at $145 \mathrm{~K}$ and $59 \mathrm{~K}$, and the $\mathrm{Nb}-\mathrm{Se}$ bonds are strongly covalent-ionic in nature. ${ }^{70-73}$ From scanning tunnelling microscopy studies of $\mathrm{NbSe}_{3}$, it is clear that there are three distinguishable chains (I, II, and III) in the $\mathrm{NbSe}_{3}$ unit cell at all temperature. ${ }^{74}$ The III chains are identified by their association with the CDW modulation vector $q^{1}$, and the two remaining chains are named I and II.

Among $\mathrm{TaX}_{3}$-based TMTCs, $\mathrm{TaS}_{3}$ and $\mathrm{TaSe}_{3}$ have been investigated due to their various fundamental microelectronic properties, which range from insulating to metallic conducting. ${ }^{65,75} \mathrm{TaS}_{3}$ was first described by Blitz and Kocher in 1938 and later through XRD studies implemented by Jellinek in 1962. ${ }^{76,77}$ There are two commonly referenced structures of $\mathrm{TaS}_{3}$, a monoclinic state $\left(\mathrm{m}-\mathrm{TaS}_{3}\right)$ and an orthorhombic state $\left(\mathrm{o}-\mathrm{TaS}_{3}\right)$ (structure shown in Fig. 6a) ${ }^{\mathbf{1 0 4}}$ (Table 1). Meerschaut et al. reported the complete structure of $\mathrm{m}-\mathrm{TaS}_{3}$ with the space group $P 2_{1} / m$, and the lattice constants are $a=9.515(2) \AA, b=3.3412(4)$ $\AA, c=14.912(2) \AA$, and $\beta=109.99^{\circ} .{ }^{75} \mathrm{O}^{-} \mathrm{TaS}_{3}$ forms in the space group $C 222_{1}$ and the lattice constants are $a=36.804 \AA, b=$ $15.173 \AA$, and $c=3.340 \AA .{ }^{65,77}$ A high-pressure $\mathrm{m}_{\mathrm{hp}}-\mathrm{TaS}_{3}$ phase with the identical space group to $\mathrm{m}-\mathrm{TaS}_{3}$ has similar lattice parameters, expect there is a deviation of $\beta$ by $3^{\circ} \cdot{ }^{65} \mathrm{~m}-\mathrm{TaS}_{3}$ is
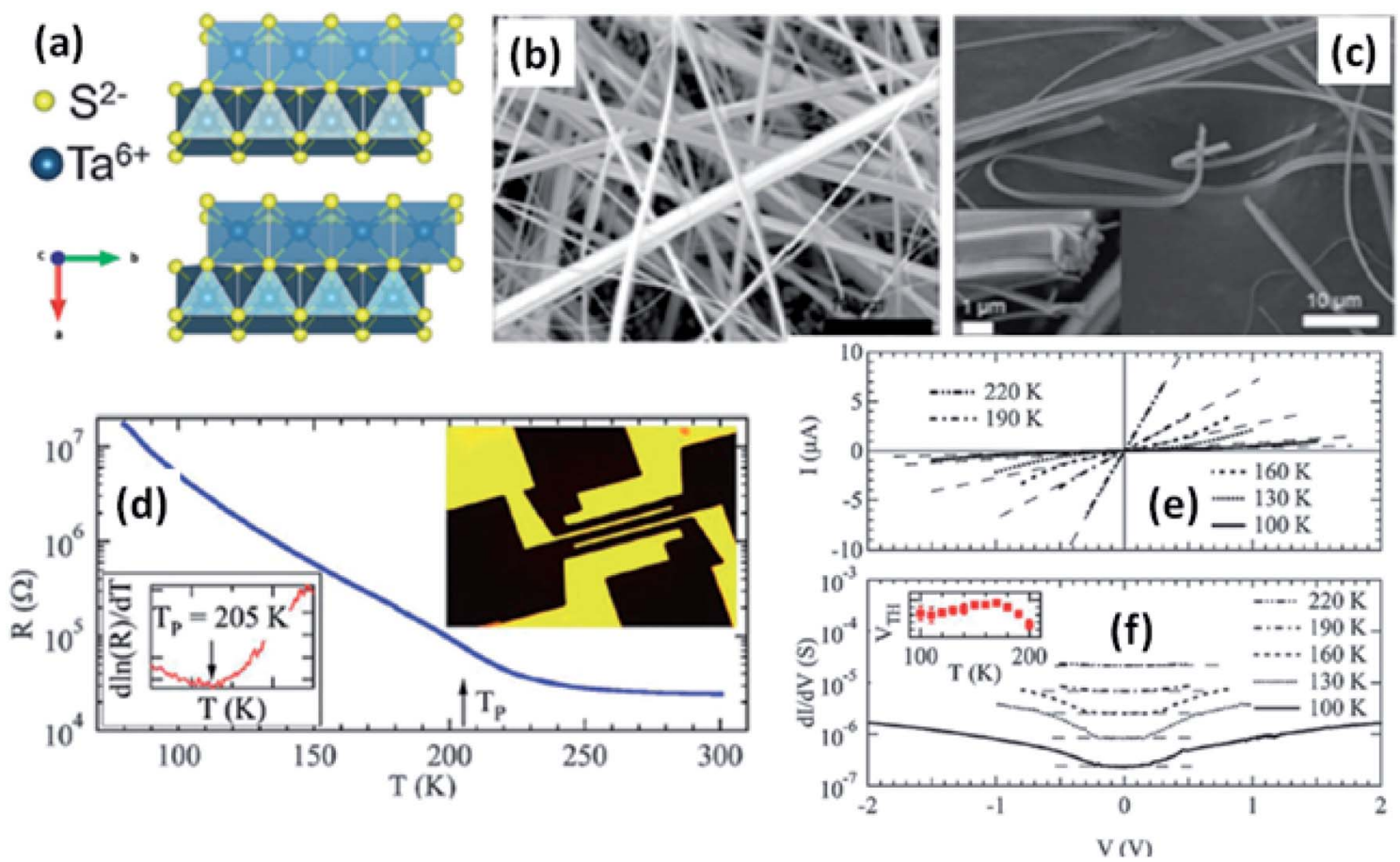

Fig. 6 (a) The orthorhombic structure of $\mathrm{TaS}_{3}$. (b and c) FESEM images of $\mathrm{TaS}_{3}$ nanobelts. (d) Temperature-dependent resistance changes of a single nanoribbon, with the inset showing a fabricated device. (e) Current-voltage curves and (f) differential conductance as a function of voltage measured at specific temperatures, with the inset of (f) showing the temperature dependence of the threshold voltage. Republished with permission from ref. 104, permission conveyed through Copyright Clearance Center, Inc. 
a low-dimensional conductor with inestimable chains of tantalum atoms along the $b$-axis of the primitive cell. The remarkable properties of bulk and few-layer $\mathrm{TaS}_{3}$ are directly correlated to the crystal structure, specifically the chains of tantalum atoms and the $b$-direction of the unit cell, which leads to quasi-1D properties. $\mathrm{TaSe}_{3}$ belongs to the $\mathrm{V}-\mathrm{X}$ group of $\mathrm{MX}_{3}$ with a monoclinic crystal structure, in which the unit cell consists of stacks of Ta atoms, each of which are fused to three Se atoms above and below the $b$-axis. ${ }^{78,136}$ The monoclinic crystal structure of $\mathrm{TaSe}_{3}$ was first recorded by Bjerkelund and Kjekshus in 1965 with $a=10.402 \AA, b=3.495 \AA, c=9.829 \AA$, and $\beta=$ $106.26^{\circ} .{ }^{79,80}$ The inter-planar distance (nearly $4 \AA$ ) is more than the distance between the Ta atoms $(3.495 \AA)$, which gives clear evidence for the quasi-1D properties of $\mathrm{TaSe}_{3} \cdot{ }^{79}$

2.2.1. Electrical, optical, and magnetic properties of $\mathrm{V}-\mathrm{X}$ type $\mathbf{M X}_{3}$. As discussed, from structural and supporting spectroscopic data analysis, the $\mathrm{V}-\mathrm{X}$ type $\mathrm{MX}_{3}$ phases can be represented by the ionic formula $\mathrm{M}^{4+}\left(\mathrm{X}_{2}\right)^{2-} \mathrm{X}^{2-}$. The dichalcogenide groups, $\left(\mathrm{X}_{2}\right)^{2-}$, are considered to be electron reservoirs and provide the $\mathrm{MX}_{3}$ phases with some astonishing properties. $\mathrm{NbS}_{3-\mathrm{I}}$ is detailed to be a semiconductor with $\mathrm{Nb}-\mathrm{Nb}\left(\mathrm{d}^{1}-\mathrm{d}^{1}\right)$ pairing in each chain. The pressure-dependent electrical properties of a quasi-one-dimensional $\mathrm{NbS}_{3}$ conductor demonstrate that whenever there is an enlargement in conductivity by six orders of magnitude at pressures of 3-4 GPa, there will be an insulator-metal transition. ${ }^{81}$ Furthermore, if the local conduction activation energy increases, then there will be an additional transition in harmony with the temperature dependence of resistance. Fedorov et al. reported the physical process and electrical properties of $\mathrm{NbX}_{3}$ thin-film FETs based on colloidal powder samples subject to ultrasonication in different solvents. ${ }^{82}$ In isopropyl-alcohol and ethanol-water mixtures, the concentrations of $\mathrm{NbSe}_{3}$ were found to be $0.332 \mathrm{~g} \mathrm{~L}^{-1}$ and $0.443 \mathrm{~g} \mathrm{~L}^{-1}$, respectively, which are the uppermost among all the used solvents. The highest carrier mobility is observed in $\mathrm{NbS}_{3}$ films obtained from the ethanol-water mixture colloidal solution [1200-2400 $\mathrm{cm}^{2} \mathrm{~V}^{-1} \mathrm{~s}^{-1}$ ], with n-type conductivity. The measured carrier mobility in $\mathrm{NbS}_{3}-\mathrm{CH}_{3} \mathrm{CN}$ colloidal solution is $\sim 10 \mathrm{~cm}^{2} \mathrm{~V}^{-1} \mathrm{~s}^{-1}$, with p-type doping. ${ }^{82}$ However, from quantum chemical studies, an understanding of electronic transitions (electron transfer from the molecular orbitals of bonding $\mathrm{Nb}-$ $\mathrm{Nb}$ bonds to anti-bonding $\mathrm{Nb}-\mathrm{Nb}$ bonds) through the excitation of $\mathrm{Nb}-\mathrm{Nb}$ bonds has allowed this to come to light as a novel concept. ${ }^{83} \mathrm{Wu}$ et al. incorporated a low concentration of $\mathrm{Ti}$ (0.05-0.18\%) into the $\mathrm{NbS}_{3-\mathrm{I}}$ host matrix, which alters the phase from triclinic to monoclinic. ${ }^{84}$ Speculative studies suggest that the phase transition can be attributed to an increase in the entire energy of the triclinic phase via p-type doping induced by titanium atoms, which have one less electron in the valance shell compared to niobium. The alloyed $\mathrm{NbS}_{3}$ preserved its crystallinity, and optical and angle-resolved Raman measurements provided information about the crystalline anisotropy and its levels. ${ }^{84}$ From polarized infrared reflection and transmission studies, the absorption co-efficient for $\mathrm{NbS}_{3-\mathrm{I}}$ was

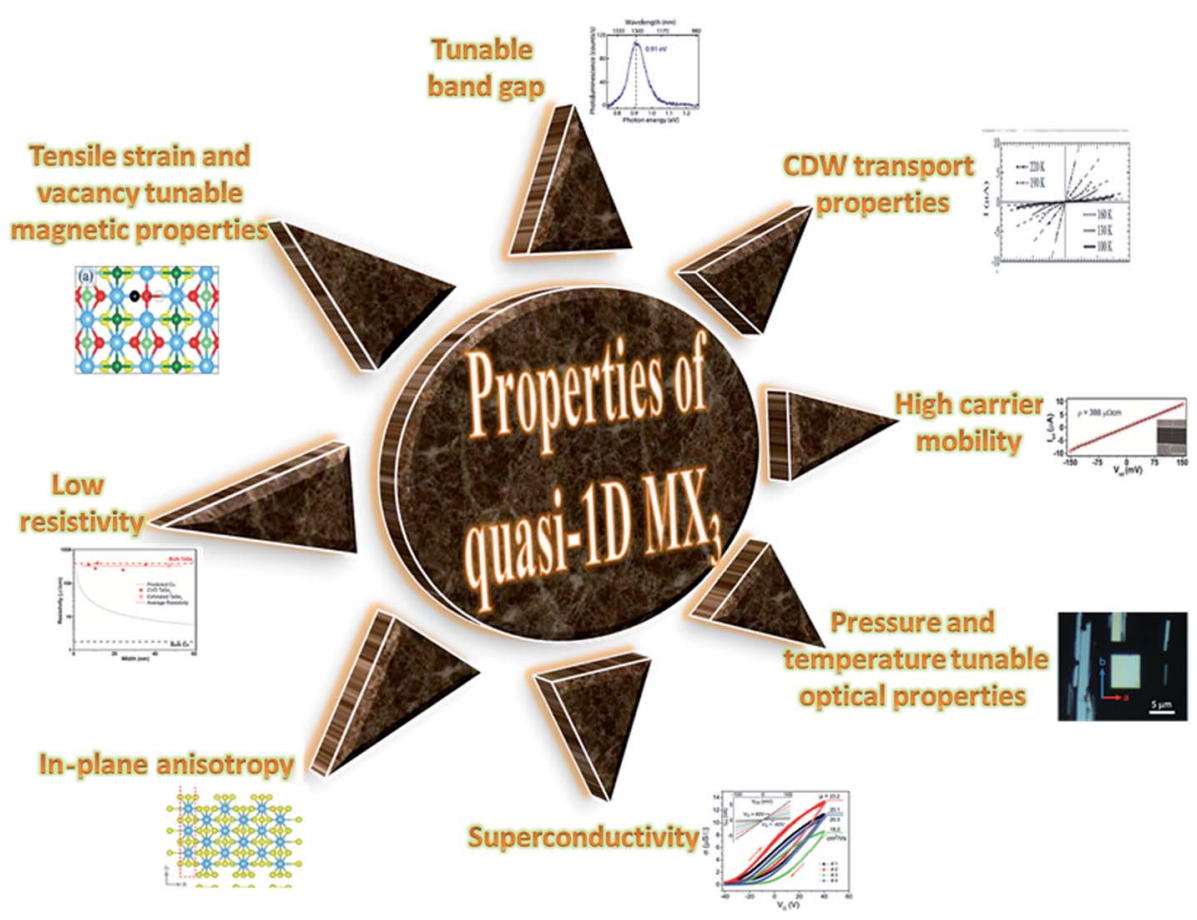

Fig. 7 A schematic diagram showing the properties of quasi-1D $M X_{3}$. Resistivity figure reprinted with permission from ref. 78, copyright: 2019, American Chemical Society; in-plane anisotropy figure republished with permission from ref. 9, permission conveyed through Copyright Clearance Center, Inc. (2016); superconductivity figure reproduced with permission from ref. 124, copyright: 2015, Royal Society of Chemistry; optical properties figure reproduced with permission from ref. 6, copyright: 2019, IOP Science; carrier mobility figure reprinted with permission from ref. 78, copyright: 2019, American Chemical Society; CDW properties figure republished with permission from ref. 104, permission conveyed through Copyright Clearance Center, Inc.; band gap figure reproduced with permission from ref. 6, copyright: 2019, IOP Science; magnetic properties figure reproduced with permission from ref. 9, copyright: 2015, American Chemical Society. 
calculated by Itkis at two different temperatures (300 and 8.5 K). ${ }^{60}$ Electron-hole excitations across the Peierls gap ${ }^{85}$ and soliton-like excitations in the superstructure led to absorption in the spectral range $>6700 \mathrm{~cm}^{-1}$ and $<6700 \mathrm{~cm}^{-1}$, respectively. ${ }^{60}$ The UV-visible absorption spectrum $(250-1000 \mathrm{~nm})$ at $300 \mathrm{~K}$ and infrared and Raman spectra at $300 \mathrm{~K}\left(650-10 \mathrm{~cm}^{-1}\right)$ and $100 \mathrm{~K}\left(650-180 \mathrm{~cm}^{-1}\right)$ of $\mathrm{NbS}_{3}$ were obtained by Sourisseau et $a l .{ }^{86}$ Their findings reveal the presence of absorbing semiconducting characteristics from UV spectroscopy, and they found 23 Raman bands and 18 infrared bands, which are close to the theoretical results. ${ }^{\mathbf{8 6}}$

Monoclinic $\mathrm{NbSe}_{3}$ consists of three equidistantly placed metal chains with different $\left(\mathrm{Se}_{2}\right)^{2-}$ groups, and it is reported to show metallic conductivity with two charge density wave temperatures of $145 \mathrm{~K}$ and 59 K. ${ }^{69,87,88}$ Chaussy et al. reported two phase-transition temperatures of $\mathrm{NbSe}_{3}$ crystals at $145 \mathrm{~K}$ and $59 \mathrm{~K}$ based on electrical resistivity, magnetic susceptibility, and heat capacity measurements. ${ }^{87}$ As per their revisions, when the temperature decreased, the resistivity $(\rho)$ decreased, showing the behaviour of a metal, and saturation was reached below $10 \mathrm{~K}$. The maximum specific heat capacity was attained at $49 \mathrm{~K}$ and gradually decreased at $T=0 \mathrm{~K}$. It was found to be diamagnetic at $4.2 \mathrm{~K}$, and for fibres and powder samples of $\mathrm{NbSe}_{3}$, the magnetization values were equal to $1.35 \times 10^{-7} \mathrm{emu}$ $\mathrm{g}^{-1}$ (attributed to a parallel orientation to the magnetic field) and $5 \times 10^{-7}$ emu g $^{-1}$ (attributed to a random orientation to the magnetic field), respectively. ${ }^{87}$ Whiskers of $\mathrm{NbSe}_{3}$ and Fe-doped $\mathrm{NbSe}_{3}$ nanowires also showed two anomalies in resistivity that were adjunct to the CDW transitions at $140 \mathrm{~K}$ and $50 \mathrm{~K}^{89}$ The successful doping of $\mathrm{Fe}$ atoms can be observed based on the strengthening of both the threshold fields, $E_{\mathrm{T}_{1}}$ and $E_{\mathrm{T}_{2}}$. Fourprobe resistivity measurements of single-crystal $\mathrm{NbSe}_{3}$ nanowires showed the expected CDW transitions at $T_{1}=142 \mathrm{~K}$ and $T_{2}=58 \mathrm{~K}$, and there was no magnetoresistance above the higher $T_{\mathrm{CDW}}$ value and positive magnetoresistance below the lower $T_{\text {CDW }}$ value. ${ }^{72}$ Ido et al. explored the effects of pressure on CDW formation and superconductivity in $\mathrm{NbSe}_{3}$ via resistivity and diamagnetic measurements. ${ }^{\mathbf{9 0}}$ It was observed that both the CDW transition temperatures $T_{1}$ and $T_{2}$ decreased steadily with an increase in pressure, while they later changed abruptly above $6 \mathrm{kbar}$ pressure and tended to zero above $P_{\mathrm{c}}=7.5 \mathrm{kbar}$ (superconductivity appeared). The change in superconductivity arose from a clampdown on electron-phonon coupling. ${ }^{\mathbf{9}}$ Latyshev et al. showed that the induction of oscillations into nonlinear CDW conductivity could be attributed to columnar defects in $\mathrm{NbSe}_{3}$, which fluctuated with a magnetic field when the field was oriented parallel to the axes of the defects. ${ }^{73,91}$ Due to the CDW transitions, there were ample signs of electronphonon scattering affecting the transport properties relating to the thermal conductivity of the lattice of $\mathrm{NbSe}_{3}$ nanowires. ${ }^{\mathbf{9 2}}$ Ong et al. performed Hall measurements of $\mathrm{NbSe}_{3}$ and showed that at both $T_{\mathrm{CDW}}$ values there is an increase in Hall resistivity $\left(R_{\mathrm{H}}\right) \cdot{ }^{93}$ But this was self-regulating below $3 \mathrm{~K}$, and with an increase in the field value, it saturated at $2.3 \times 10^{-6} \mathrm{~m}^{3} \mathrm{C}^{-1}$. Surprisingly, the value of $R_{\mathrm{H}}$ was $4.1 \times 10^{-7} \mathrm{~m}^{3} \mathrm{C}^{-1}$ at $2 \mathrm{~K}^{93}$

Resistivity measurements and electron diffraction studies of $\mathrm{m}-\mathrm{TaS}_{3}$ and $\mathrm{o}-\mathrm{TaS}_{3}$ showed two transition temperatures $(240 \mathrm{~K}$ and $160 \mathrm{~K}$ ), which were interpreted as succeeding Peierls transitions on the diverse chain types of $\mathrm{TaS}_{3} \cdot{ }^{94}$ For o-TaS 3 , as the temperature dropped below the ambient temperature, the resistance gradually rose up to $230 \mathrm{~K}$ and then increased sharply. However, for $\mathrm{m}-\mathrm{TaS}_{3}$, this happened in a typical manner, i.e., there was a sequential decrease and increase in resistance at 270,220 , and $180 \mathrm{~K}^{94}$ The Peierls transition temperature, the temperature at which the slope of $R v s$. T attains its maximum, was also shown by Roucau et al. Peierls transitions in quasi-1D conductors are associated with intrinsic superstructures. ${ }^{94}$ Polarized Raman scattering studies of o-TaS demonstrated that at the Fermi surface, the formation of a CDW gap was held responsible for the reduction in free carriers. ${ }^{95}$ Thus, the scattering intensity decreased from interband processes along with an increase in the phonon energy. Nanosized $\mathrm{TaS}_{3}$ samples showed step-like conductivity as a function of strain, demonstrating the association of the steps with the quantization of the CDW wave vector. ${ }^{96}$ In $\mathrm{o}^{\mathrm{T}} \mathrm{TaS}_{3}$, the asymmetrical conductivity had no dependency below a certain temperature $(2 \mathrm{~K})$, which was caused by soliton transport in the CDW system..$^{97}$ Nichols et al. studied the frequency and voltage dependencies of voltage-induced torsional strain in o-TaS $_{3}$ and concluded that the strain is allied with a divergence in the CDW instead of the CDW current. ${ }^{98}$ A change in the length, $L$, (depending on the electric field and time) of $\mathrm{TaS}_{3}$ samples demonstrated that hysteresis partly corresponds with resistance. ${ }^{99}$ Thermal expansion studies of $\mathrm{o}-\mathrm{TaS}_{3}$ crystals showed the uncharacteristic behaviour of the hysteresis loop of length $L$ below the Peierls transition temperature and at $100 \mathrm{~K}$, the elastic modulus meets the Young's modulus of the CDW wave. ${ }^{100}$ Gorlova and co-workers observed electric-field-induced torsional strain corresponding to colossal shear in $\mathrm{TaS}_{3}$ whiskers. ${ }^{\mathbf{1 0 1}}$ The threshold and hysteresis behaviour of torsion demonstrated its link with CDW deformation. Correspondingly, Nichols et al. investigated the effects of hysteretic voltageinduced torsional strain on CDW depinning in o-TaS $\mathrm{T}_{3}$ using square-wave and triangular-wave voltages of dissimilar frequencies and amplitudes. ${ }^{\mathbf{1 0 2}}$ Inagaki et al. reported the magnetoresistance of a $\mathrm{CDW}$ in $\mathrm{O}^{-\mathrm{TaS}_{3}}$ whiskers under a magnetic field up to $4.2 \mathrm{~K}$. When the field was aligned with the $a$-axis, the maximum amplitude of angle-dependent magnetoresistance was achieved, whereas there was zero value at the $b$ axis. ${ }^{103}$ Electrical transport measurements of single o-TaS $\mathrm{S}_{3}$ by Farley et al. discovered the depression of the Peierls transition temperature to $205 \mathrm{~K}$. Below this temperature, there was depinning of the CDW, which was accredited to broadening of the electric field and surface confinement; low dimensionality and a limited size effect caused a great enhancement in the threshold voltage for the nucleation of CDW dislocations (Fig. 6). ${ }^{104} \mathrm{Wu}$ et al. studied the pressure-dependent vibrational properties, which imply that the one and only $\mathrm{S}_{\|}$mode (at $54 \mathrm{~cm}^{-1}$ ) was held accountable for the crystalline orientation of $\mathrm{TaS}_{3}$ through angle-resolved Raman spectroscopy and highpressure diamond anvil cell studies. ${ }^{\mathbf{1 0 5}}$ Frequency-dependent conductivity measurements showed the Drude type behaviour of the inertial feedback of $\mathrm{TaS}_{3}$, associated with damping. ${ }^{106}$ 


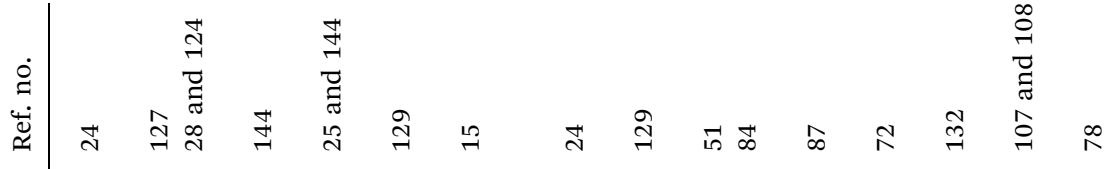

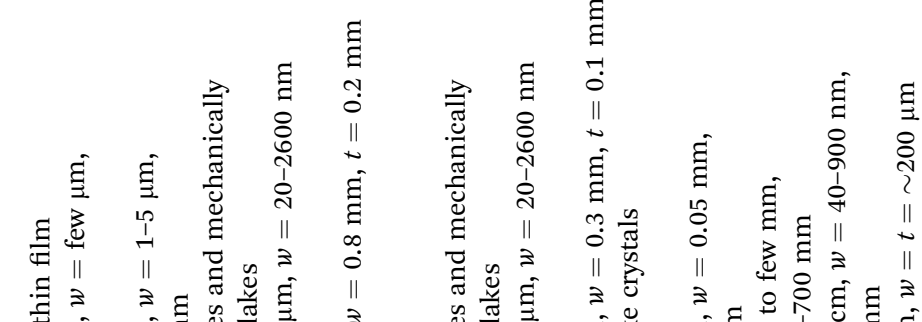

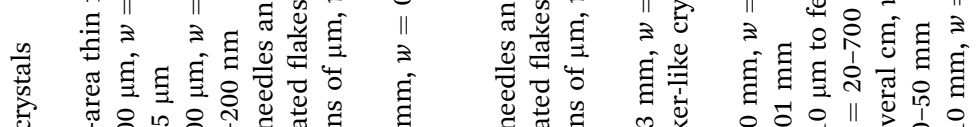

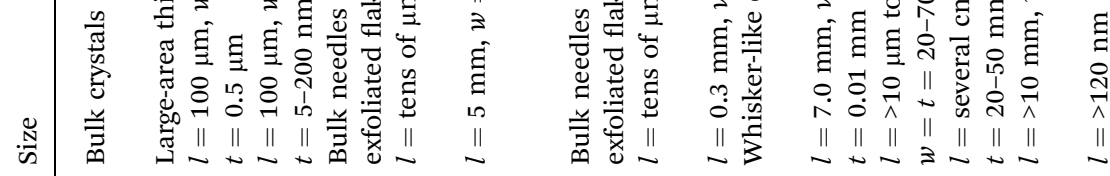

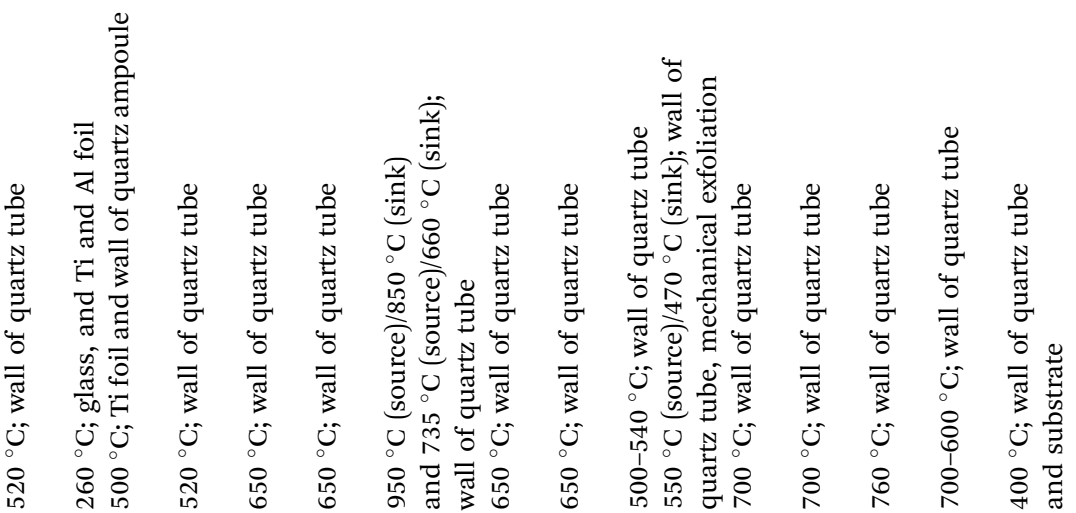

$$
\begin{aligned}
& \text { 焉 }
\end{aligned}
$$

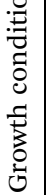

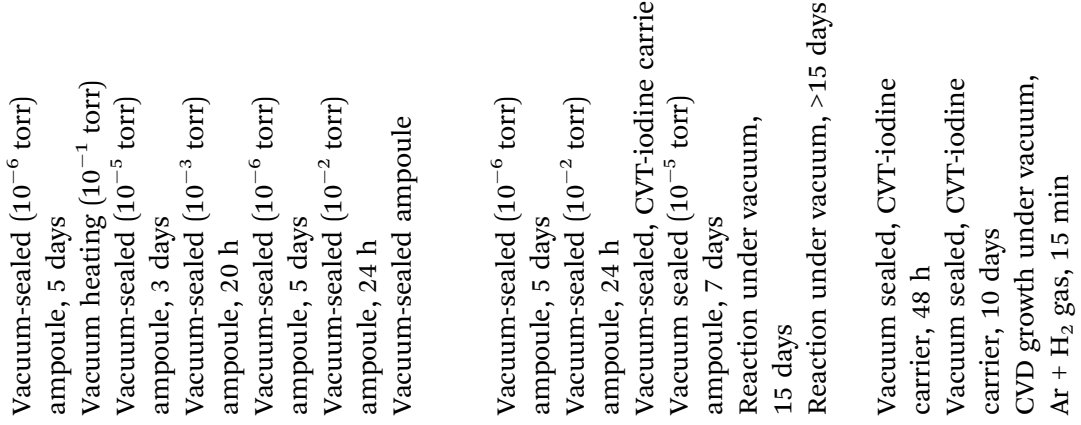

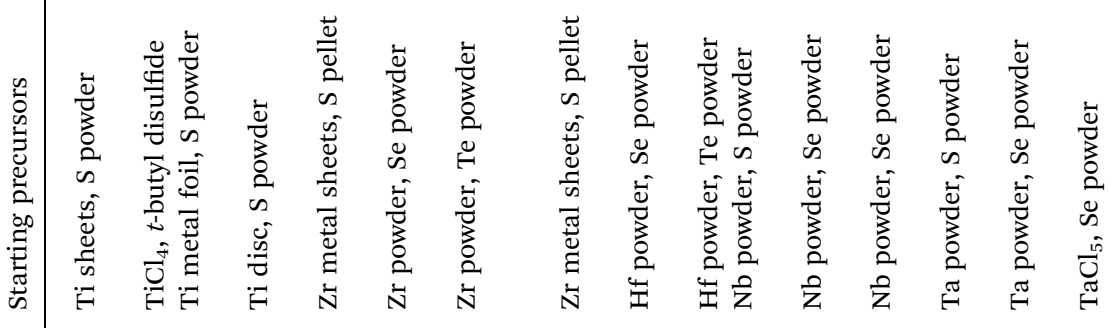

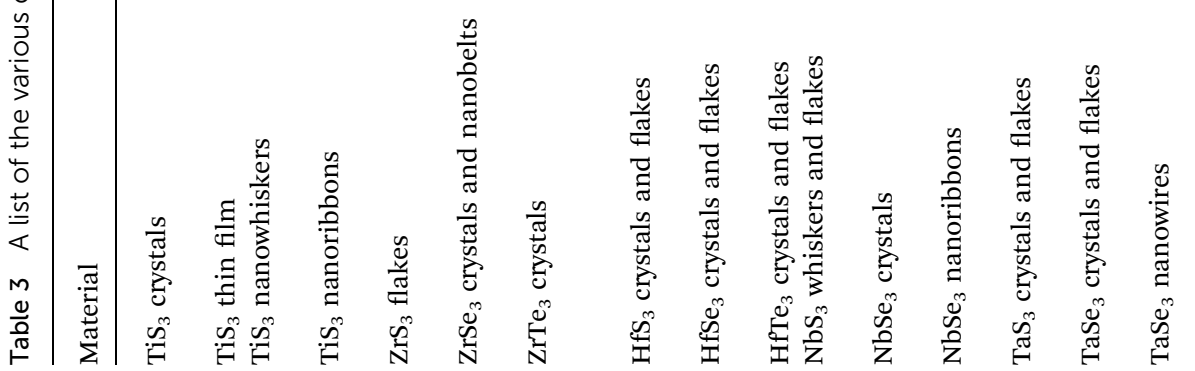


$\mathrm{TaSe}_{3}$ is reported to be superconducting below $2.1 \mathrm{~K}$ with anisotropic properties under the application of a magnetic field ${ }^{79}$ and Kikkawa et al. also indicated the superconductivity of $\mathrm{TaSe}_{3}$ at $T=1.9 \mathrm{~K}^{65}$ Thickness-dependent work function variations of $\mathrm{TaSe}_{3}$ flakes confirmed the extended screening length $(24 \mathrm{~nm})$ at which the work function suddenly decreased, which specified its hydrophilic nature, and the Raman modes at $128 \mathrm{~cm}^{-1} ; 141,164,217$, and $237 \mathrm{~cm}^{-1} ; 177 \mathrm{~cm}^{-1}$; and $186 \mathrm{~cm}^{-1}$ corresponded to $\mathrm{B}_{\mathrm{g}}, \mathrm{B}_{2}, \mathrm{~A}_{\mathrm{g}}, \mathrm{A}_{1 \mathrm{~g}}$ modes, respectively. ${ }^{107} \mathrm{TaSe}_{3}$ is reported to have a high current-carrying capacity and high current density, and it has emerged as a possible potential candidate to act as an interconnector in electronic devices due to capping with hexagonal boron nitride (h-BN). ${ }^{108}$ Experimental observations demonstrated that quasi-1D $\mathrm{TaSe}_{3}$ nanowires transmit lower levels of normalized noise spectral density, with potential for rationalized local interdependent applications. ${ }^{109-111}$ The main properties of $\mathrm{MX}_{3}$ are summed up in Fig. 7.

\section{Strategies for the growth of $M X_{3}$}

Synthesis approaches used for the growth of $\mathrm{MX}_{3}$ crystals and nanostructures such as nanowhiskers, nanoribbons, nanosheets, nanowires, etc. can be classified as top-down or bottomup approaches. In this review, we have emphasized different synthesis approaches reported for $\mathrm{MX}_{3}$ development, as summarized in Table 3. Useful methods for $\mathrm{MX}_{3}$ include direct chemical reactions, chemical vapour transport (CVT), chemical vapour deposition (CVD), high-pressure evolution approaches, intercalation, mechanical and chemical exfoliation, etc. Characterization techniques such as X-ray diffraction (XRD), X-ray photoelectron spectroscopy (XPS), field-emission scanning electron microscopy (FESEM), atomic force microscopy (AFM), scanning tunnelling microscopy (STM), transmission electron microscopy (TEM), Raman spectroscopy, UV spectroscopy, and optical microscopy (angle-resolved as well as pressure- and temperature-dependent methods) are the usual procedures used to estimate the properties of $\mathrm{MX}_{3}$ materials and further help in exploring their abundant applications. ${ }^{\mathbf{1 1 2 - 1 2 0}}$

\subsection{Bottom-up approaches}

3.1.1. IV-X-type $\mathbf{M X}_{\mathbf{3}}$. Transition-metal trichalcogenides nanostructures, such as nanowhiskers, nanoribbons, and nanosheets, are reported to be grown based on direct reactions involving titanium and sulphur., 121-126 In a typical reaction process for the growth of $\mathrm{TiS}_{3}$ crystals and nanostructures, Ti and $S$ are sealed in an evacuated quartz ampoule and heated up to $500-600{ }^{\circ} \mathrm{C}$ for several days (3-5 days). ${ }^{124}$ In this approach, the sulfurization temperature and gradient used usually are $\sim 500{ }^{\circ} \mathrm{C}$ and $50-100{ }^{\circ} \mathrm{C}$, respectively. This method is also termed as a chemical vapour transport (CVT) technique, which is an excellent growth mechanism for fabricating lowdimensional materials, where sulphur, $\operatorname{TiS}_{x}$ species, and sometimes iodine are considered as transport agents. ${ }^{2}$ Fig. $8^{\mathbf{1 2 4}}$ shows photographs of ampoules with Ti foil and S powder used for the direct reaction-based method to grow bare and doped $\mathrm{TiS}_{3}$ nanowhiskers. Fig. $8 \mathrm{c}$ and $\mathrm{d}^{\mathbf{1 2 4}}$ show SEM images of nanowhiskers grown on $\mathrm{Ti}$ foil and the walls of the quartz tube. ${ }^{\mathbf{1 2 4}}$ It is reported that the critical temperature for the CVT growth of $\mathrm{TiS}_{3}$ should be below a typical temperature, i.e., $632{ }^{\circ} \mathrm{C}$, which is the decomposition temperature of $\mathrm{TiS}_{3}$ to $\mathrm{TiS}_{2}$ (Table 3). $\mathrm{TiS}_{3}$ nanoribbons and nanosheets have been fabricated via mixing Ti powder with sulphur gas through a solid-gas reaction. The sulphur powder was heated in a vacuum sealed ampoule at $550{ }^{\circ} \mathrm{C}$ (nanoribbons) and $400{ }^{\circ} \mathrm{C}$ (nanosheets) for around 15 days. ${ }^{27} \mathrm{TiS}_{3}$ thin films are prepared via thermal chemical vapour deposition through reacting $\mathrm{TiCl}_{4}$ and $t$-butyl disulphide (TBDS) at $260{ }^{\circ} \mathrm{C}^{\mathbf{1 2 7}}$ The temperature and pressure of the chamber, sulfurizing source, and flow rates of $\mathrm{Ar}$ and $\mathrm{TiCl}_{4}$ play crucial roles in achieving the formation of pure $\mathrm{TiS}_{3}$ films. Similar to the CVT growth approach for $\mathrm{TiS}_{3}, \mathrm{ZrS}_{3}$ and $\mathrm{HfS}_{3}$ can also be grown via taking $\mathrm{Zr}$ and $\mathrm{Hf}$ elemental sheets and sulphur powder and heating them in vacuum sealed quartz ampoules at $650{ }^{\circ} \mathrm{C}$ for 5 days. ${ }^{24,128} \mathrm{ZrS}_{3}$ bulk powder is reported to form upon heating zirconium and sulphur powder in an

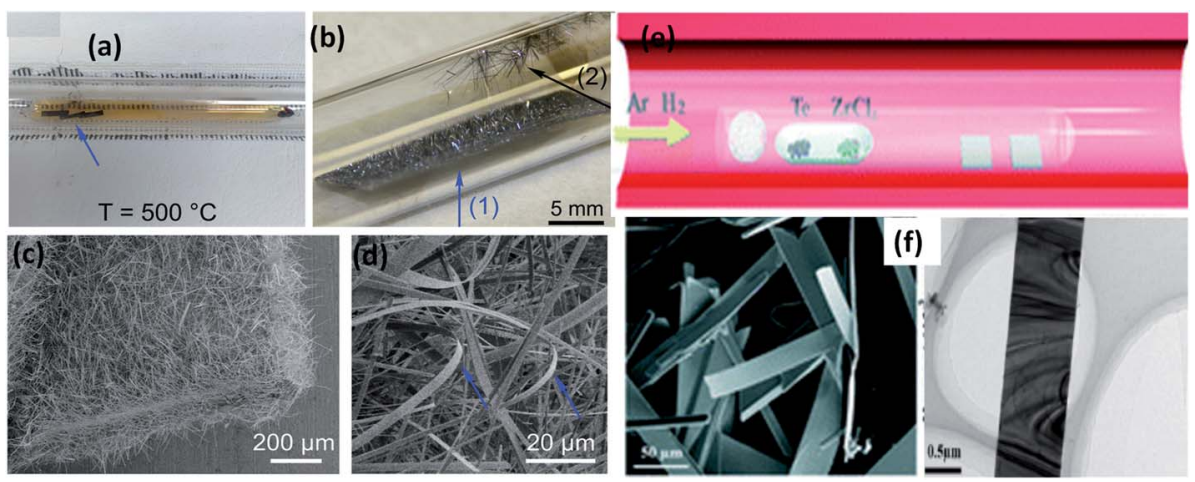

Fig. 8 The synthesis of $M X_{3}$. Optical photographs of the ampoule (a) before and (b) after the growth of TiS 3 whiskers on Ti foil and the surface of quartz. ( $c$ and d) SEM images of the grown $\mathrm{TiS}_{3}$ whiskers with arrow marks indicating samples collected from the respective positions. Reproduced with permission from ref. 124, copyright: 2015, Royal Society of Chemistry. (e) The synthesis of ZrTe 3 via a chemical vapour deposition approach. (f) FESEM and TEM images of $\mathrm{ZrTe}_{3}$. Republished with permission from ref. 130, permission conveyed through Copyright Clearance Center, Inc. 
evacuated quartz ampoule for $150 \mathrm{~h}$, and a stable colloidal dispersion could be formed in different organic media, such as iso-propanol, acetonitrile, ethanol, and dimethyl formamide, via a liquid exfoliation process. ${ }^{35} \mathrm{ZrSe}_{3}$ and $\mathrm{HfSe}_{3}$ nanobelts could be grown via a CVT approach, in which Zr or Hf powder was mingled with Se powder at a stoichiometric ratio $(1: 3) .{ }^{129}$ The powder mixture was vacuum sealed $\left(10^{-2} \mathrm{~Pa}\right)$ in a quartz tube and heated at $650{ }^{\circ} \mathrm{C}$ for $24 \mathrm{~h}$ to achieve the growth of $\mathrm{ZrSe}_{3}$ and $\mathrm{HfSe}_{3}$ nanobelts. $\mathrm{ZrTe}_{3}$ nanoribbons could be grown via a CVD approach, in which Te and $\mathrm{ZrCl}_{4}$ powder are used as vapour sources (Fig. 8e and f). ${ }^{130}$ Through controlling the carrier gas flow (Ar: $30 \mathrm{sccm}$ and $\mathrm{H}_{2}: 35 \mathrm{sccm}$ ), heating temperature $\left(650-750{ }^{\circ} \mathrm{C}\right)$, and growth time (20-80 $\left.\mathrm{min}\right)$, high-quality and single-crystal nanoribbons could be grown on a $\mathrm{SiO}_{2} / \mathrm{Si}$ substrate placed downstream of the quartz tube. ${ }^{\mathbf{1 3 0}}$

3.1.2. V-X-type $\mathbf{M X}_{3}$. The growth of $\mathrm{NbX}_{3}$ - and $\mathrm{TaX}_{3}$-based transition metal trichalcogenides has been reported using similar CVT approaches as followed for the IV-X $\mathrm{MX}_{3}$ group, and some of the results are summarized in Table 3. Whiskerlike $\mathrm{NbSe}_{3}$ nanowires were prepared via the direct reaction of a $\mathrm{Nb}$ and Se powder mixture placed in a small alumina crucible, sealed under vacuum and heated for 24 h. ${ }^{89}$ Hor et al. reported an effective single-step approach for the synthesis of $\mathrm{NbSe}_{3}$ nanowires and nanoribbons. ${ }^{72}$ In this approach, stoichiometric quantities of $\mathrm{Nb}$ and Se were sealed and heated in a quartz ampoule at $630-700{ }^{\circ} \mathrm{C}$ to achieve the growth of pure and crystalline nanoribbons. ${ }^{89}$ Pham et al. reported a facile method to prepare few-to-single chain structures of $\mathrm{NbSe}_{3}$, encapsulated in BN or CNT sheaths to prevent oxidation. ${ }^{\mathbf{1 3 1}}$ In this approach, $\mathrm{Nb}$ and Se powder was mixed with cap-opened CNTs/BNNTs and vacuum sealed at $10^{-1}$ torr in a quartz ampoule, followed by heating at $690{ }^{\circ} \mathrm{C}$ for 5-9 days. Wu et al. reported the preparation of $\mathrm{TaS}_{3}$ nanobelts at $760^{\circ} \mathrm{C}$ for $48 \mathrm{~h}$ in a quartz ampoule via a CVT approach, with iodine as the transport agent. ${ }^{\mathbf{1 3 2}}$ Single crystals of o-TaS 3 are reported to be formed via a CVT approach upon heating Ta sheets and $\mathrm{S}$ powder in a sealed quartz tube at $530{ }^{\circ} \mathrm{C}$ for a few weeks. ${ }^{103}$ Long nanofibers of $\mathrm{o}-\mathrm{TaS}_{3}$ can be prepared via heating Ta and $\mathrm{S}$ precursors in a sealed quartz ampoule at $500{ }^{\circ} \mathrm{C}$ for 48 h. ${ }^{134}$ Farley et al. prepared o-TaS nanoribbons via heating Ta foil and S powder at $550{ }^{\circ} \mathrm{C}$ for $2 \mathrm{~h}$ in a vacuum-sealed tube. ${ }^{\mathbf{1 0 4}}$ The sublimed sulphur travelled across the tube due to a temperature gradient inside the tube and reacted with $\mathrm{TaS}_{3}$. o-TaS 3 whiskers were prepared via a CVT approach in a quartz ampoule through maintaining two heating zones at $650{ }^{\circ} \mathrm{C}$ and $550{ }^{\circ} \mathrm{C}$ in a tube furnace for 7 days. ${ }^{105} \mathrm{In}$ a similar approach, Li et al. reported the generation of large-
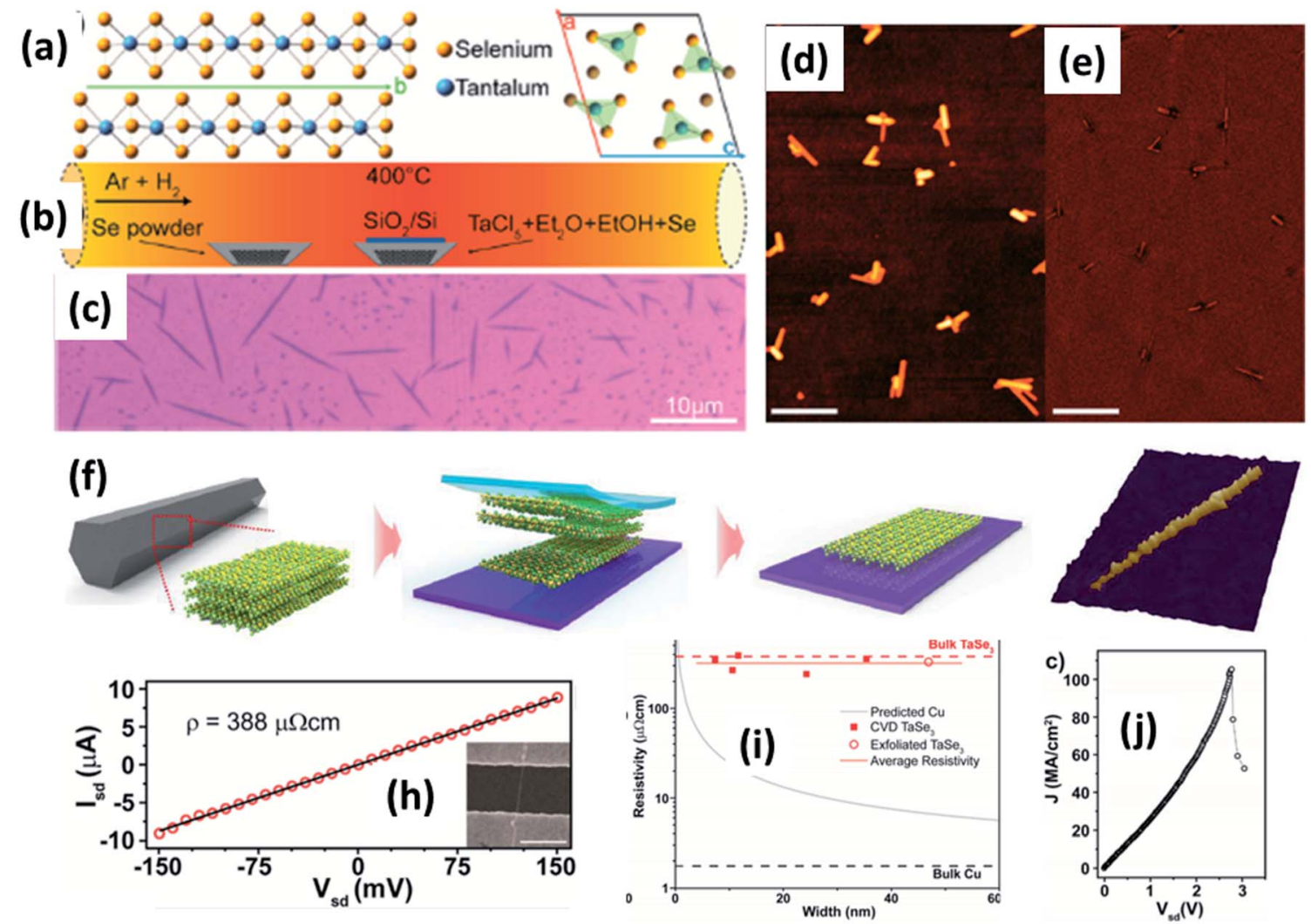

Fig. 9 (a) The crystallographic structure of $\mathrm{TaSe}_{3}$. (b) A schematic diagram of the CVD set-up for the growth of TaSe 3 nanowires. (c) An optical image and ( $d$ and e) SEM images of a population of TaSe 3 nanowires. Reprinted with permission from ref. 78, copyright: 2019, American Chemical Society. (f) A schematic illustration of the mechanical exfoliation of $\mathrm{TaSe}_{3}$ flakes from bulk TaSez. (g) A 3D representation of a quasi-1D TaSe 3 nanoribbon monolayer on a $\mathrm{SiO}_{2} / \mathrm{Si}$ substrate. Reproduced with permission from ref. 107, copyright: 2019, Multidisciplinary Digital Publishing Institute. (h) Source-drain current vs. voltage for a $11.6 \mathrm{~nm} \mathrm{TaSe}$ nanowire in a 2-electrode configuration, with the inset showing an SEM image of the device. (i) Resistivity as a function of bundle width of CVD-grown TaSe 3 nanowires and a comparison with bulk Cu and exfoliated TaSe ${ }_{3}$. (j) The current density response of a $7 \times 7 \mathrm{~nm}^{2}$ nanowire as a function of $V_{\mathrm{ds}}$ with failure at a current density of $108 \mathrm{~A} \mathrm{~cm}$. Reprinted with permission from ref. 78, copyright: 2019, American Chemical Society. 


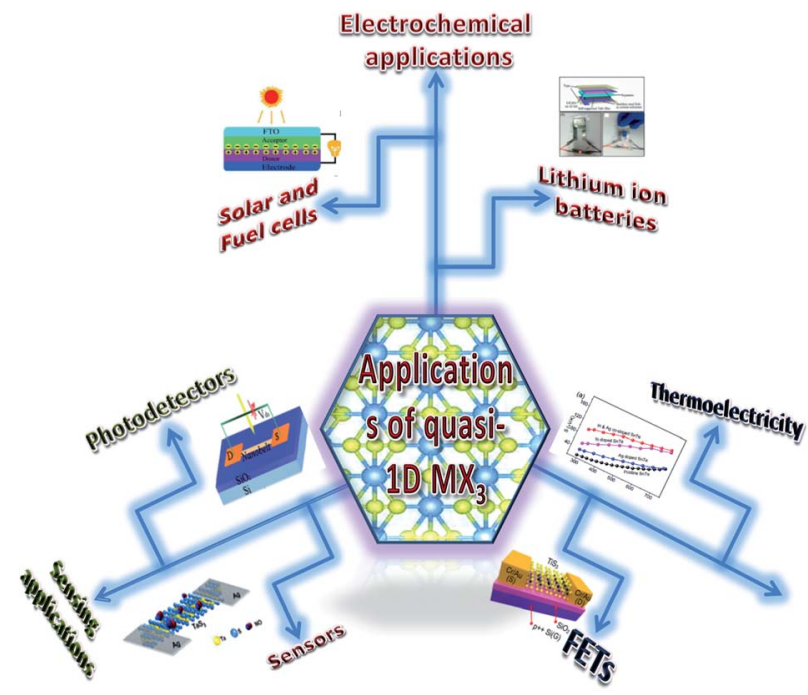

Fig. 10 A schematic diagram showing the various applications of $M X_{3}$. Solar and fuel cell figure republished with permission from ref. 33 permission conveyed through Copyright Clearance Center, Inc.; lithium-ion batteries figure reprinted with permission from ref. 134 copyright: 2015, American Chemical Society; sensors figure reprinted with permission from ref. 133, copyright: 2018, American Chemical Society; photodetectors figure republished with permission from ref. 129, permission conveyed through Copyright Clearance Center, Inc.; FETs figure reproduced with permission from ref. 124, copyright: 2015 , Royal Society of Chemistry; thermoelectricity figure republished with permission from ref. 157, permission conveyed through Copyright Clearance Center, Inc.; central figure reproduced with permission from ref. 6, copyright: 2015, Springer Nature.

scale and self-supported $\mathrm{TaS}_{3}$ nanowires via the direct reaction of Ta and S powder in a sealed quartz tube at $650{ }^{\circ} \mathrm{C}$ for $1 \mathrm{~h} \cdot{ }^{134} \mathrm{To}$ prepare $\mathrm{TaSe}_{3}$ crystals, Ta and Se precursors are heated under vacuum at a pressure of $1 \mathrm{GPa}$ at $500-700{ }^{\circ} \mathrm{C}$ for $30-240$ min. ${ }^{135}$ Stolyarov et al. followed a two-step CVT approach; in the first step, a Ta, Se, and iodine mixture was heated in a quartz ampoule at $900{ }^{\circ} \mathrm{C}$ for $12 \mathrm{~h}$, and in the second step, the product was heated in a tube furnace with a temperature gradient of 700-680 ${ }^{\circ} \mathrm{C} .{ }^{108,110}$ In the CVD approach, $\mathrm{TaCl}_{5}$ and Se powder samples were used as the reactants and heated at $400{ }^{\circ} \mathrm{C}$ for 15 min with $\mathrm{Ar}$ and $\mathrm{H}_{2}$ as ambient process gases to grow $\mathrm{TaSe}_{3}$ nanowires on $\mathrm{SiO}_{2} / \mathrm{Si}$ placed upstream near an alumina crucible filled with $\mathrm{TaCl}_{5}$ (Fig. 9a-e). ${ }^{78}$ In another work, Kim et al. prepared single-crystal $\mathrm{TaSe}_{3}$ via a CVT approach, mixing stoichiometric amounts of $\mathrm{Ta}, \mathrm{Se}$, and iodine powder and heating this at $670{ }^{\circ} \mathrm{C}$ for 10 days, maintaining the growth zone at $600{ }^{\circ} \mathrm{C}$, and later mechanically exfoliating it from bulk $\mathrm{TaSe}_{3}$ (Fig. 9f-g). ${ }^{107}$ Empante et al. reported low resistivity and a high collapse current density of $10^{8} \mathrm{~A} \mathrm{~cm}^{-2}$ for a single nanowire, which featured an electro-migration energy barrier twice that of $\mathrm{Cu}(\text { Fig. } 9 \mathrm{~h}-\mathrm{j})^{78}$

\subsection{Top-down approaches}

Chemical and mechanical exfoliation methods are widespread synthesis tactics that have gained huge interest in the field of top-down approaches. ${ }^{137,138}$ Mostly, as-prepared bulk crystals are taken as a prime precursor and subsequent chemical intercalation or power-driven force approaches are applied to these. Then, through force or intercalation, the neighbouring layers get detached from adjacent layers. Weak van der Waals forces co-existing between neighbouring layers plays a vibrant role in the materialization of few- to mono-layer crystals of $2 \mathrm{D}$ materials upon controlling the relevant parameters. Similarly, the ultrasonication of bulk powder or crystals in solvents is widely
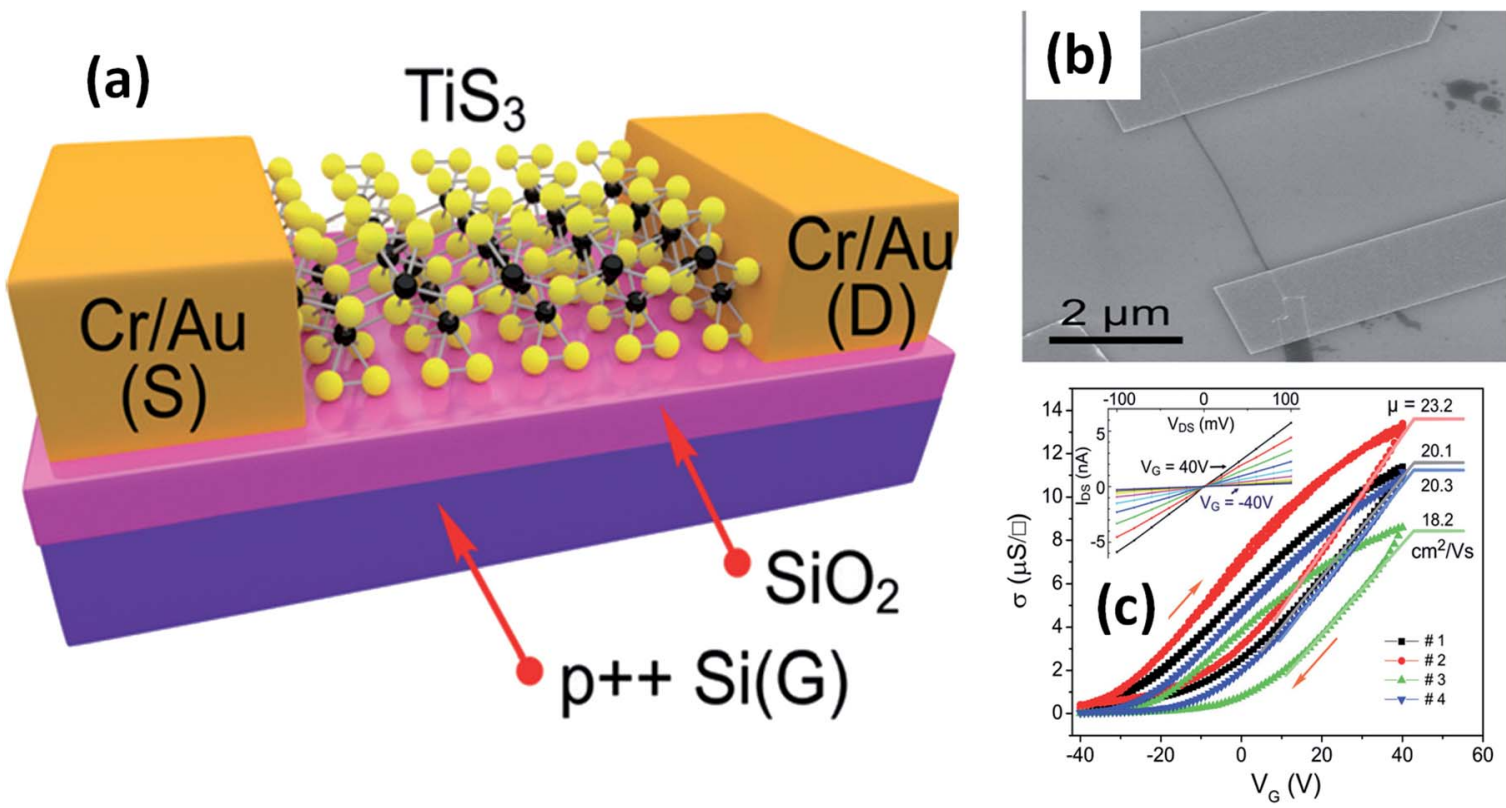

Fig. 11 Few-layered TiS 3 FETs. (a) A schematic diagram and (b) an SEM image of a typical FET. (c) Conductivity vs. gate voltage dependencies of four different fabricated FETs, with the inset showing drain source current vs. drain source voltage at different gate voltages. Reproduced with permission from ref. 124, copyright: 2015, Royal Society of Chemistry. 

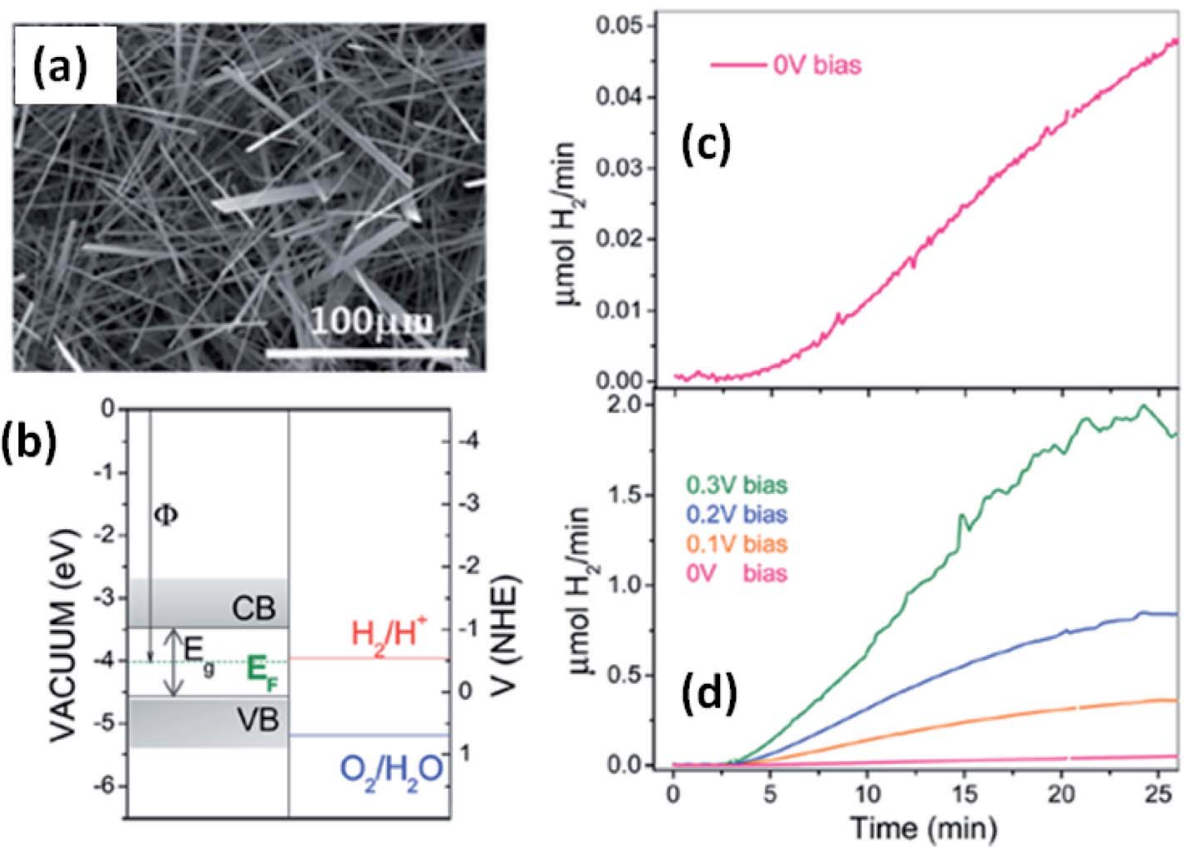

Fig. 12 The hydrogen photogeneration properties of $M X_{3}$ nanostructures. (a) An SEM image and (b) the conduction and valence band energy levels on potential ( $\mathrm{V} v$ s. NHE) and energy (eV vs. vacuum) scales, with the redox potentials for the water-splitting half reactions at $\mathrm{pH}=9.0 \mathrm{vs}$. $\mathrm{NHE}$, of $\mathrm{TiS}_{3}$ nanoribbons. The hydrogen evolution flow of $\mathrm{TiS}_{3}$ nanoribbons (c) at $0.0 \mathrm{~V}$ and (d) at different bias potentials. Republished with permission from ref. 144, permission conveyed through Copyright Clearance Center, Inc.

used for liquid exfoliation to achieve single- to few-layered nanosheets.

Mechanical exfoliation is a widely used method to isolate single- to few-layered $\mathrm{MX}_{3}$ nanosheets from bulk material. ${ }^{6}$ On the other hand, liquid phase exfoliation is an effective method for the large-scale production of $\mathrm{MX}_{3}$ nanosheets. ${ }^{139} \mathrm{Few}$-layer $\mathrm{TiS}_{3}$ material was prepared by Island $e t$ al. at a temperature of $400{ }^{\circ} \mathrm{C}$ with sheet-like morphology, and this was later (a)

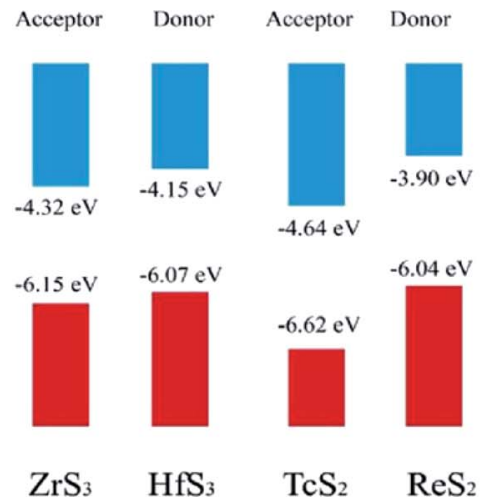

(c)

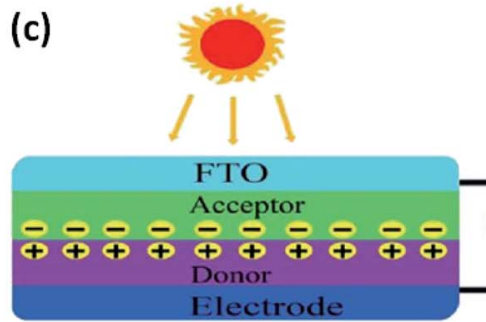

(b)

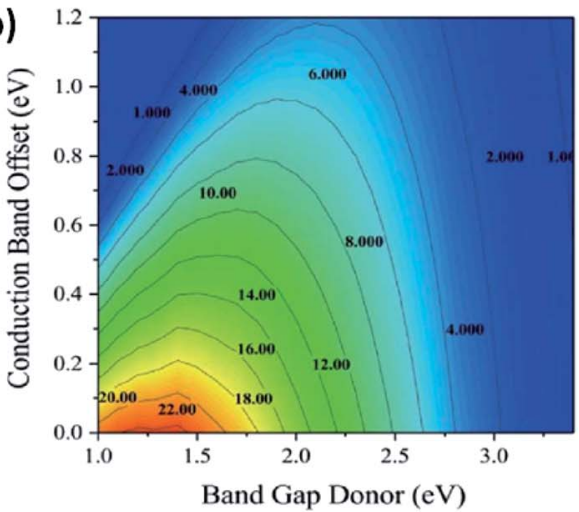

Donor

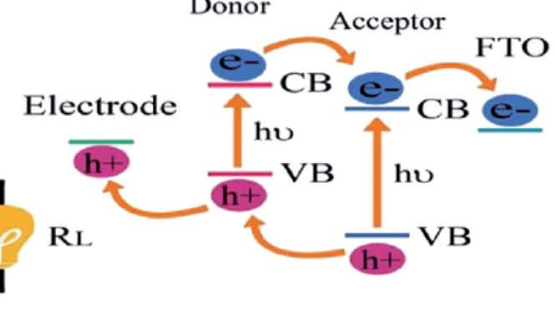

Fig. 13 The application of $\mathrm{MX}_{3}$ heterostructures to solar cells. (a) Band offsets of $\mathrm{ZrS}_{3} / \mathrm{HfS}_{3}$ and $\mathrm{TcS}_{2} / \mathrm{ReS}_{2}$ with the vacuum level as zero reference. (b) PCE contours obtained as a function of the donor band gap and conduction band offset. (c) A schematic illustration of thin-film solar cells with the associated mechanism. Republished with permission from ref. 33, permission conveyed through Copyright Clearance Center, Inc. 
mechanically exfoliated to few-layer form. ${ }^{6}$ Xie et al. reported the liquid phase exfoliation of bulk $\mathrm{ZrS}_{3}$ in $n$-propylamine for $30 \mathrm{~min}$ followed by heating at $120^{\circ} \mathrm{C}$ for 3 days in a Teflon-lined autoclave. ${ }^{139}$ The obtained product was washed and ultrasonicated in 1-cyclohexyl-2-pyrrolidinone to achieve $\mathrm{ZrS}_{3}$ nanosheets. $\mathrm{NbS}_{3}$ and $\mathrm{NbSe}_{3}$ nanoparticle colloidal solutions were prepared via a top-down approach through the ultrasonication of powder in different solutions (DMF, acetone, acetonitrile, ethanol, a water-ethanol mixture, etc.) for $3 \mathrm{~h}$. Liion intercalation is proposed as a suitable method to prepare $\mathrm{MX}_{3}$ nanoribbons, in which three lithium atoms are incorporated into an $\mathrm{MX}_{3}$ unit to yield $\mathrm{Li}_{3} \mathrm{MX}_{3}{ }^{140} \mathrm{~A}$ mechanical exfoliation approach is employed to prepare few-layered flakes from $\mathrm{TaSe}_{3}$ crystals (Fig. 9f and g). ${ }^{\mathbf{1 0 7}}$ A schematic diagram of the different steps used in this process is shown in Fig. 9, in which wafer dicing tape is used and stuck onto the crystal, which is then removed and adhered to the $\mathrm{SiO}_{2} / \mathrm{Si}$ substrate.

\section{Advanced applications of $M X_{3}$}

\subsection{FETS}

The possible applications of TMTCs are shown in Fig. 10. Due to the direct optical band gap $(\sim 1 \mathrm{eV})$ and ultra-high response of $\mathrm{TiS}_{3}$, it has been used as an appropriate material for field-effect transistors with high gain. ${ }^{\mathbf{1 2 5 , 1 2 6}}$ FETs based on 2D sheets of $\mathrm{TiS}_{3}$ whiskers (mechanically exfoliated few-layered samples) have been fabricated on $\mathrm{SiO}_{2} / \mathrm{Si}$ substrates (Fig. 11a and b). ${ }^{124}$ n-Type electronic transport $\mathrm{TiS}_{3}$-based FETs exhibited mobilities of 18$24 \mathrm{~cm}^{2} \mathrm{~V}^{-1} \mathrm{~s}^{-1}$, and this was improved to $43 \mathrm{~cm}^{2} \mathrm{~V}^{-1} \mathrm{~s}^{-1}$ upon the addition of another substrate, i.e., $\mathrm{Al}_{2} \mathrm{O}_{3}$, through
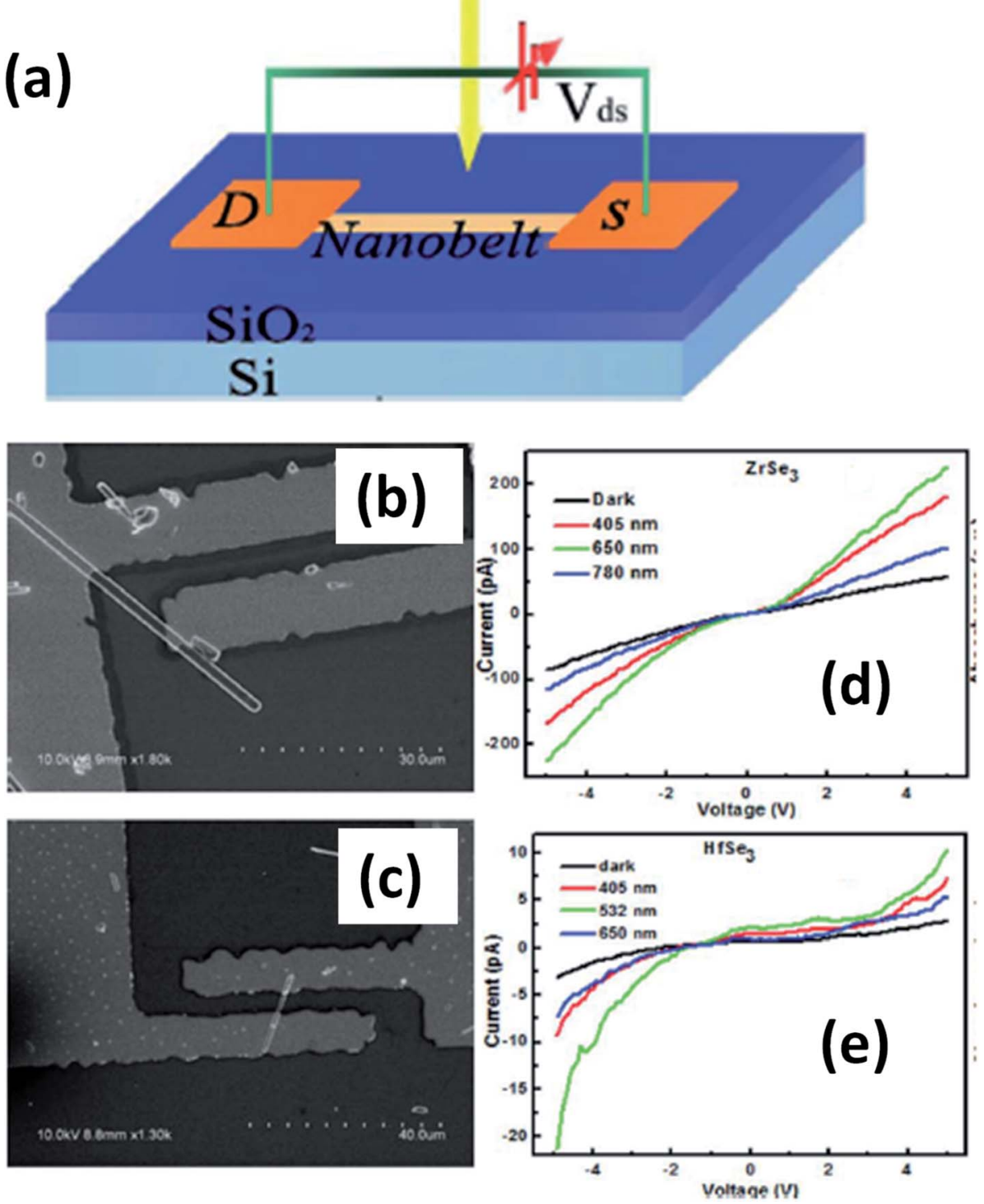

Fig. 14 (a) A schematic illustration of a $\mathrm{ZrSe}_{3}$ and $\mathrm{HfSe}_{3}$ single nanobelt photodetector. SEM images of (b) $\mathrm{ZrSe}_{3}$ and (c) the $\mathrm{HfSe}_{3}$ photodetector. I-V characteristics of (d) $\mathrm{ZrSe}_{3}$ and (e) the $\mathrm{HfSe}_{3}$ nanobelt photodetector. Republished with permission from ref. 129, permission conveyed through Copyright Clearance Center, Inc. 
a conventional atomic layer deposition (ALD) procedure. Similarly, the ON/OFF ratio improved to 7000 from 300 upon the use of $\mathrm{Al}_{2} \mathrm{O}_{3}$ as an alternative substrate (Fig. 11c). ${ }^{124}$ The temperaturedependent transfer curves of $\mathrm{TiS}_{3}$ nanowire FETs showed a metal-insulator transition, with a cross-over temperature of 220 $\mathrm{K}$ and mobility of $20-30 \mathrm{~cm}^{2} \mathrm{~V}^{-1} \mathrm{~s}^{-1} \cdot{ }^{141}$ To demonstrate the anisotropic electrical properties, a $\mathrm{TiS}_{3}$ nanosheet FET device was made up, with electrodes at $30^{\circ}$ intervals. ${ }^{\mathbf{1 2 6}}$ The fabricated FET device worked along the $b$-axis of the $\mathrm{TiS}_{3}$ nanosheets, which was determined via computing the transfer characteristics across the device between two opposite electrodes at variable angles. A polar plot of current $v s$. voltage showed the high mobility of $80 \mathrm{~cm}^{2} \mathrm{~V}^{-1}$ $\mathrm{s}^{-1}$ (b-axis) and low mobility of $40 \mathrm{~cm}^{2} \mathrm{~V}^{-1} \mathrm{~s}^{-1}$ (a-axis) of the nanosheets. Transfer characteristics curves showed n-type behaviour, with an ON/OFF ratio of five. ${ }^{\mathbf{1 2 6}}$ The nanoribbons showed lower mobilities with advanced electric field and optical properties, which later can be tuneable. The multifaceted concentration of sulphur vacancies in samples grown at lower temperature played an important role in creating tuneable properties upon an intensification in the n-type dopant. ${ }^{\mathbf{1 2 6}}$ The interfacial and contact properties of multi-layered $\mathrm{TiS}_{3}$ and metals ( $\mathrm{Au}, \mathrm{Ag}, \mathrm{Pd}, \mathrm{Pt}$, Ir, and Ni) have been inspected via DFT and the outcomes suggested the absence of tunnelling barriers, demonstrating superior carrier injection abilities from the metal to multilayer $\mathrm{TiS}_{3}$ in FET and other electrical devices. ${ }^{\mathbf{1 4 2}}$ The output characteristics of FETs based on $\mathrm{HfS}_{3}$ nanobelts confirmed the p-type semiconducting nature. ${ }^{142}$ Therefore, from the above data, it is quite evident that these materials are first and foremost highly suitable materials for nanoelectronics and optoelectronic devices.

\subsection{Solar and fuel cell devices}

Solar water splitting using photoelectrochemical cells is an effective and efficient way of storing solar energy in the form of hydrogen, which can be used as a fuel. For hydrogen generation via water splitting, the light absorbing material with appropriate energy band positions, i.e., the energy band levels (CB and $\mathrm{VB}$ ), should be suitable with respect to the water reduction potential. ${ }^{143,163}$ Anisotropic $\mathrm{MX}_{3}$ materials possess the advantages of having a low band gap to absorb direct solar energy and
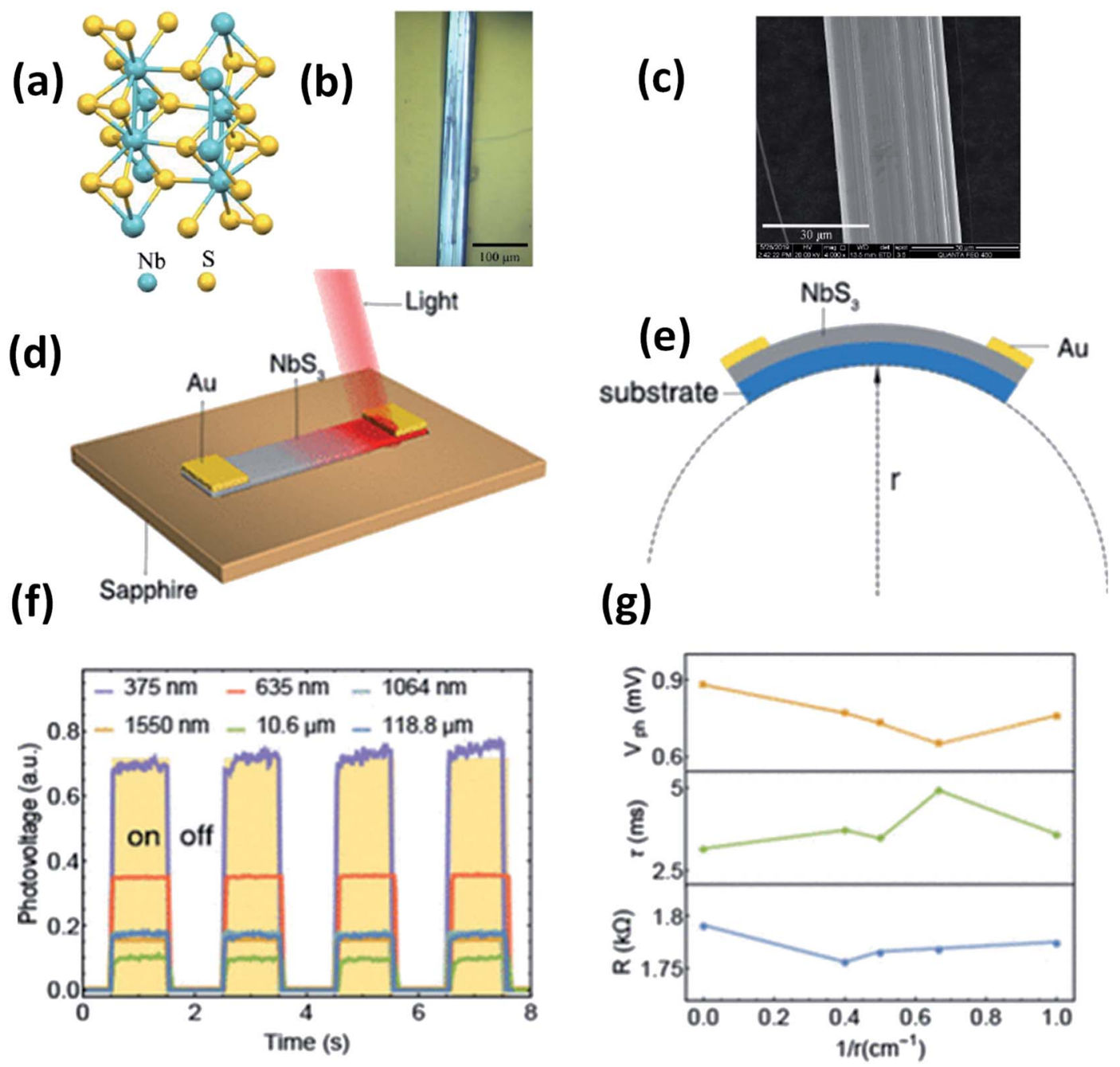

Fig. 15 (a) The crystal structure of $\mathrm{NbS}_{3}$. (b) Optical microscopy and (c) SEM images of a NbS 3 crystal. Schematic diagrams of (d) a NbS 3 photodetector and (e) bending conditions. (f) ON-OFF photovoltage curves at room temperature and (g) resistance, response time, and photovoltage data under different bending conditions. Reprinted with permission from ref. 151, copyright: 2020, American Chemical Society. 
being widely available, non-toxic, and suitable for photoelectrochemical water splitting. Fig. $12 \mathrm{a}^{\mathbf{1 4 4}}$ shows an SEM image of $\mathrm{TiS}_{3}$ nanoribbons, which have been employed as an active material for electrochemical water splitting. ${ }^{\mathbf{1 4 4}}$ The systematic energy level band diagram of $\mathrm{TiS}_{3}$ /electrolyte $\left(\mathrm{Na}_{2} \mathrm{SO}_{3}\right)$ is shown in Fig. $12 \mathrm{~b}^{\mathbf{1 4 4}}$ with a flat band potential $\left(V_{\mathrm{fb}}\right)$ at $-0.48 V_{\mathrm{NHE}}$, and this is used to calculate the semiconductor Fermi level. The conduction and valence band energy levels in terms of potential and energy balance, along with the redox potentials for the water splitting half reactions at $\mathrm{pH}=9$, are shown in the band diagram. Hydrogen evolution occurs on the $\mathrm{TiS}_{3}$ nanoribbons at $0 \mathrm{~V}$ and investigations at different bias potentials yielded a photoconversion efficiency of about $7 \%$ at a bias potential of $0.3 \mathrm{~V}$ (Fig. 12c and d). ${ }^{\mathbf{1 4 4}}$ In another work, Flores et al. demonstrated the energy level schemes for an $\mathrm{MX}_{3} /$ electrolyte interface, and comparable photogenerated hydrogen fluxes are reported for $\mathrm{TiS}_{3}, \mathrm{ZrS}_{3}$, and $\mathrm{HfS}_{3}{ }^{145} \mathrm{~A} \mathrm{TiS}_{3}$ photoanode is reported to generate up to $19 \mathrm{nmol} \mathrm{H}_{2}$ per min per $\mathrm{cm}^{2}$ at an external bias potential of $0.3 \mathrm{~V} v$ s. $\mathrm{Ag} / \mathrm{AgCl}$. Due to the presence of copious amounts of $\mathrm{Se}_{2}$ bonds on the surface of the $\mathrm{ZrS}_{3}$ ultrathin nanosheets, enhanced oxygen evolution reaction performance was observed compared to the bulk counterpart. ${ }^{139}$ A low onset overpotential of $244 \mathrm{mV}$ and Tafel slope of $45 \mathrm{mV}$ per decade are achieved using $\mathrm{ZrS}_{3}$ nanosheets in strongly alkaline solution $(\mathrm{pH}=14)$, whereas in weakly alkaline solution $(\mathrm{pH}=6.9)$, the onset over-potential and Tafel slope are reported to be lower. ${ }^{139}$

The use of 2D materials and their heterojunctions in optoelectronics and solar-correlated devices is utterly controlled by the superiority of the heterojunction formed and the band alignment to tune carriers at interfaces. ${ }^{33}$ Zhao et al. employed DFT to calculate the band superstructures and heterostructures of IV-VIA monolayers of $\mathrm{MX}_{3}(\mathrm{M}=\mathrm{Zr}, \mathrm{Hf} ; \mathrm{X}=\mathrm{S}$, Se $)$ and VIIBVIA monolayer $\mathrm{MX}_{2}(\mathrm{M}=\mathrm{Tc}, \mathrm{Re} ; \mathrm{X}=\mathrm{S}$, Se). The calculations indicated that for $\mathrm{MX}_{3}$, the valence bands are dependent on the p-states of chalcogens, whereas the d-states of the transition metals control the conduction bands. For $\mathrm{MX}_{2}$ monolayers, both the valence and conduction bands depend on the d-states of the transition metals. Considering standard water redox potentials $\left(-4.44 \mathrm{eV}\right.$ and $-5.67 \mathrm{eV}$ for reduction $\left(\mathrm{H}^{+} / \mathrm{H}_{2}\right)$ and oxidation $\left(\mathrm{O}_{2} / \mathrm{H}_{2} \mathrm{O}\right)$, respectively), the combination of $\mathrm{MX}_{3}$ and $\mathrm{MX}_{2}$ monolayers and their band alignment to create efficient heterostructures have been reported. ${ }^{33}$ Fig. $13^{33}$ shows the bandoffset components and a contour map of power conversion efficiency (PCE) values. From calculations, it is predicted that a $\mathrm{ZrS}_{3} / \mathrm{HfS}_{3}$ bilayer thin-film device could achieve $16-18 \%$ efficiency, which is much higher than other reported 2D heterojunction solar cell devices. Similarly, Ahammed et al. reported that the PCEs in $\mathrm{ZrS}_{3} / \mathrm{MoS}_{2}, \mathrm{ZrS}_{3} / \mathrm{WS}_{2}, \mathrm{ZrS}_{3} / \mathrm{MoSeTe}, \mathrm{ZrS}_{3} / \mathrm{WSTe}$, and $\mathrm{ZrS}_{3}$ /WSeTe heterostructured bilayers are as high as $\sim 12 \%$, $8 \%, 16 \%, 14 \%$, and $14 \%$, respectively. ${ }^{146}$ Recently, anisotropic $\mathrm{ZrS}_{3}$ has been reported to act as an active material for perovskite light-emitting diodes and $\mathrm{P}-\mathrm{N}$ junction diodes, and the results are found to be promising, which opens up pathways for further research into the area of $\mathrm{MX}_{3}$ heterojunctions for optoelectronics and solar-cell devices. ${ }^{147,148}$

\subsection{Photodetectors and sensors}

Island et al. reported the photoresponse properties of $\mathrm{TiS}_{3}$ nanoribbon-based FETs. ${ }^{125} \mathrm{TiS}_{3}$ FETs showed a high photoresponse of up to $2910 \mathrm{~A} \mathrm{~W}^{-1}$, and fast switching times of $\sim 4$ ms, with a cut-off frequency of $100 \mathrm{~Hz}$, showing promise for photodetection and photovoltaic applications. Tao et al. reported flexible a visible light photodetector formed on $\mathrm{ZrS}_{3}$
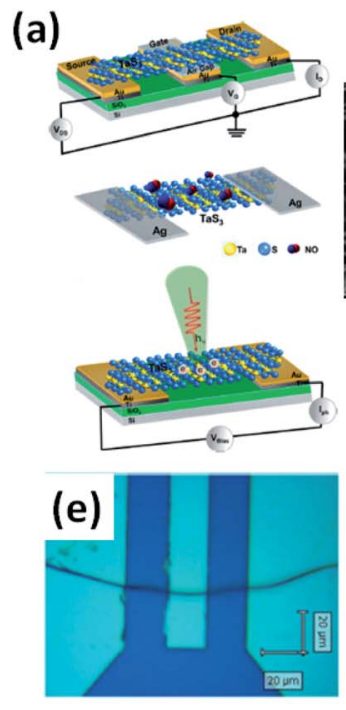
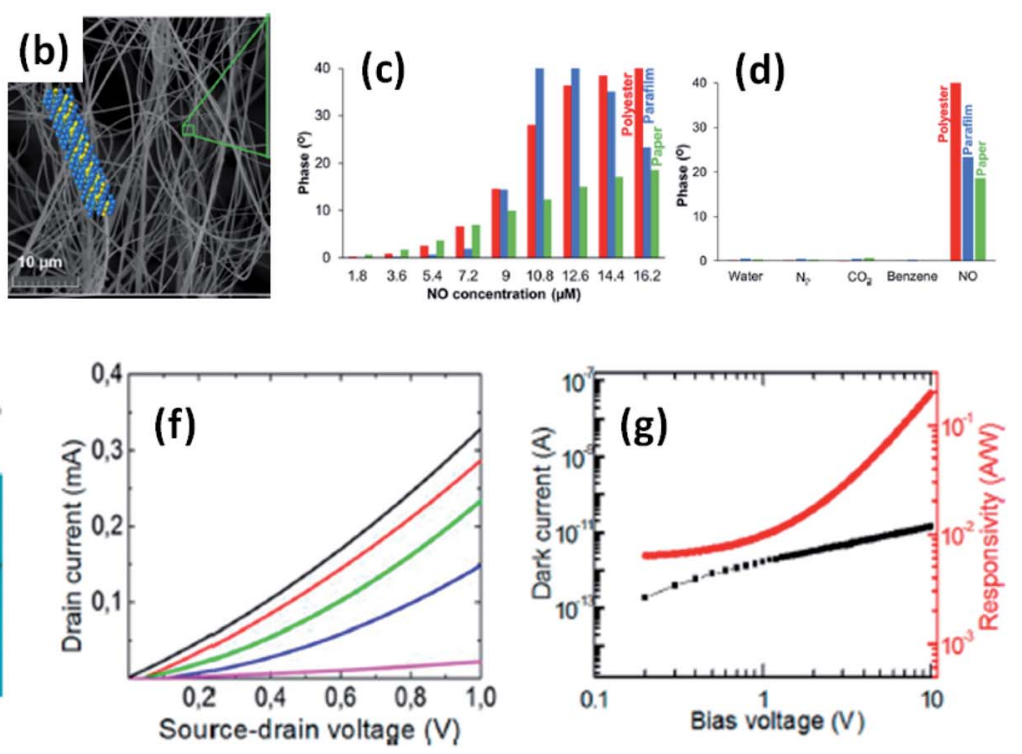

Fig. 16 (a) Schematic representations of a FET, NO gas sensor, and photodetector based on $\mathrm{TaS}_{3}$ nanofibers. (b) An SEM image of the TaS 3 nanofibers. (c) The impedance phase responses of gas sensors fabricated on different substrates (polyester, parafilm, paper) as functions of NO concentrations. (d) Selectivity studies of the sensors fabricated on different substrates. (e) An optical image of a FET based on TaS 3 fibers. (f) FET transistor characteristic curves at different gate voltages (pink: $-1 \mathrm{~V}$, blue: $0 \mathrm{~V}$, green: $1 \mathrm{~V}$, red: $2 \mathrm{~V}$, black: $3 \mathrm{~V}$ ). (g) Dark current (black) and responsivity (red) curves. Reprinted with permission from ref. 133, copyright: 2018, American Chemical Society. 
nanobelt film, which showed high spectral selectivity, a wide range, and a rapid photoresponse in the visible light to nearinfrared region. ${ }^{149}$ Photodetectors based on $\mathrm{HfS}_{3}$ FETs showed a huge ON/OFF ratio of 337.5, along with an ultralow dark current of $0.04 \mathrm{pA}$ at $405 \mathrm{~nm}$ under $1.2 \mathrm{~mW} \mathrm{~cm}^{-2}$ light excitation. ${ }^{32,142}$ CVT-deposited $\mathrm{ZrSe}_{3}$ and $\mathrm{HfSe}_{3}$ were investigated for photodetection applications. ${ }^{129}$ Under excitation by $650 \mathrm{~nm}$ wavelength light, a $\mathrm{ZrSe}_{3}$ photodetector showed a light ON/OFF ratio of 1.92 at $50 \mathrm{~s}$ with a bias voltage of $5 \mathrm{~V}$ (Fig. 14). ${ }^{129}$ Similarly, $\mathrm{a} \mathrm{HfSe}_{3}$ nanobelt photodetector showed an ON/OFF ratio of 2.2 with an average time of $50 \mathrm{~s}$, and the photoresponse time was $0.4 \mathrm{~s}$. Although $\mathrm{HfTe}_{3}$-based materials are reported to show interesting properties, including CDWs, superconductivity, and the ability to be used in quantum Hall-effect-related devices, they have been less studied to date. ${ }^{51,150} \mathrm{Wu}$ et al. reported a photothermoelectric (PTE) detector based on $\mathrm{NbS}_{3}$ with considerable performance in the $\mathrm{UV}$ to terahertz range. ${ }^{\mathbf{1 5 0}}$ Considering its immense surface-to-volume ratio and reduced magnitude, mechanically exfoliated quasi-1D $\mathrm{NbS}_{3}$ crystals prepared via a CVT approach were studied for photodetector applications (Fig. 15a-d). ${ }^{151}$ Various types of continuous-wave lasers were used to test the photoresponse performance, including UV (375 nm), near-infrared (NIR, 1064 and $1550 \mathrm{~nm}$ ), visible $(635 \mathrm{~nm})$, and semiconductor lasers, a mid-infrared (MIR, $10.6 \mu \mathrm{m}) \mathrm{CO}_{2}$ laser, and a terahertz-wave-generating farinfrared gas laser $(118.8 \mu \mathrm{m})$ (Fig. 15e). ${ }^{151}$ The photodetector based on $\mathrm{NbS}_{3}$ flakes showed good performance, with responsivities higher than $1 \mathrm{~V} \mathrm{~W}^{-1}$, a response time of $\sim 7 \mathrm{~ms}$, and robust flexibility and stability (Fig. 15e-g). ${ }^{151}$ Via employing the interesting structural and attractive electronic properties of layered $\mathrm{TaS}_{3}$ prepared in the form of nanofibers, Pumera and co-workers reported a highly selective impedimetric NO gas sensor, FET and photodetector (Fig. 16). ${ }^{133}$ The nanofibers showed metallic character, with a metal-semiconducting transition below $210 \mathrm{~K}$, prompted by CDW formation. The impedimetric gas sensor showed an excellent response towards NO when fabricated on different substrates (Fig. 16). ${ }^{133} \mathrm{~A} \mathrm{TaS}_{3^{-}}$ based sensor fabricated on a polyester substrate showed the best sensing performance, with a limit of detection (LOD) of $0.48 \mathrm{ppb}$.
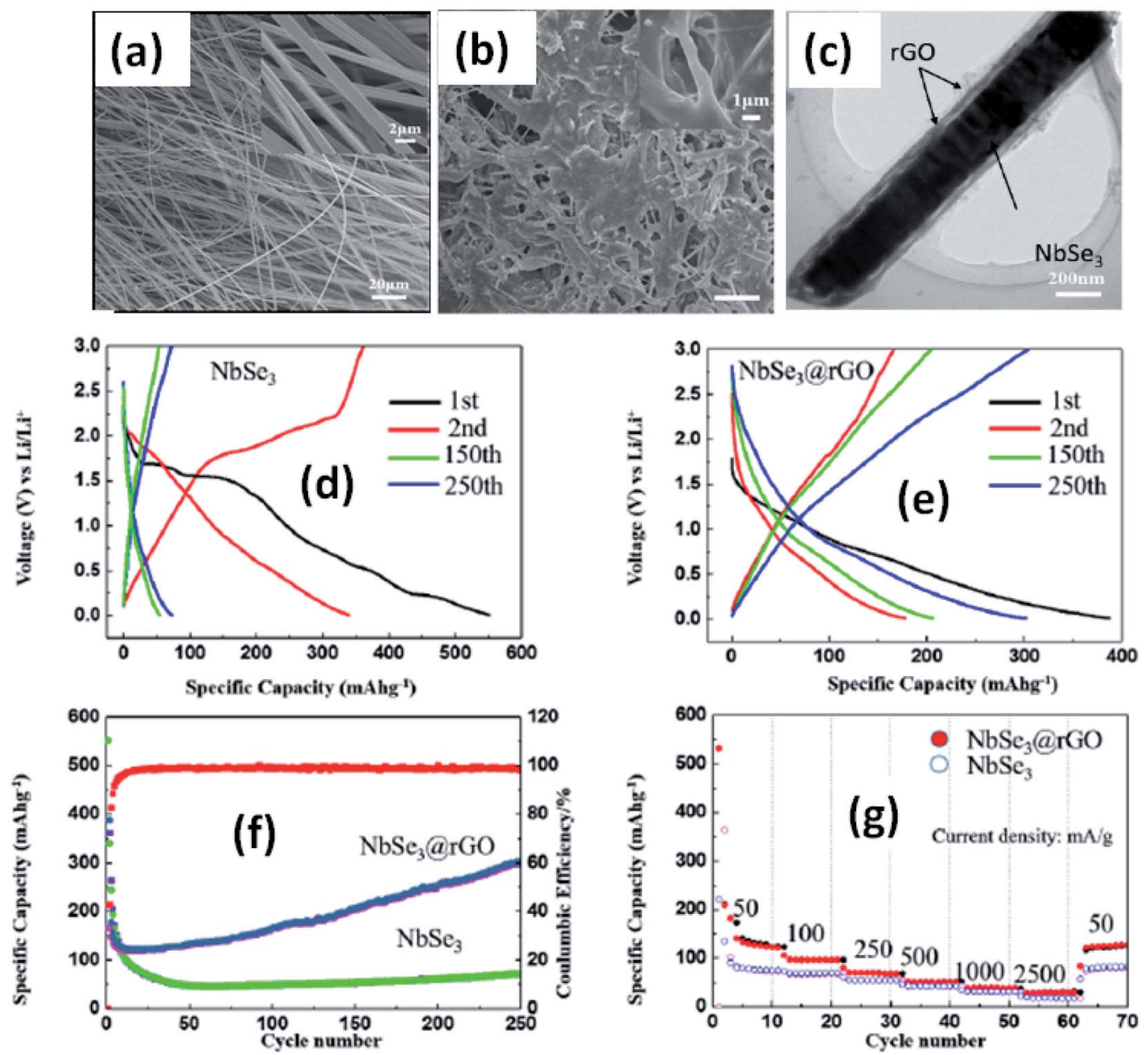

Fig. $17 \mathrm{SEM}$ images of (a) $\mathrm{NbSe}_{3}$ and (b) $\mathrm{NbSe}_{3} \mathrm{arGO}$. (c) A TEM image of $\mathrm{NbSe}_{3} \mathrm{arGO}$. The electrochemical performance of NbSe $e_{3}$ and $\mathrm{NbSe}_{3} \mathrm{QrGO}$ in the voltage range of 0.005-3 V vs. Li/Li ${ }^{+}$. Discharge and charge curves of (d) NbSe $\mathrm{N}_{3}$ and (e) nanobelts. (f) The cycling performance of $\mathrm{NbSe}_{3} \mathrm{arGO}$ and $\mathrm{NbSe}_{3}$ nanobelts at a current density of $100 \mathrm{~mA} \mathrm{~g}{ }^{-1}$. (g) The rate performances of $\mathrm{NbSe}_{3} \mathrm{arGO}$ and $\mathrm{NbSe}_{3}$ nanobelts. Reprinted from ref. 155, copyright: 2017, with permission from Elsevier. 

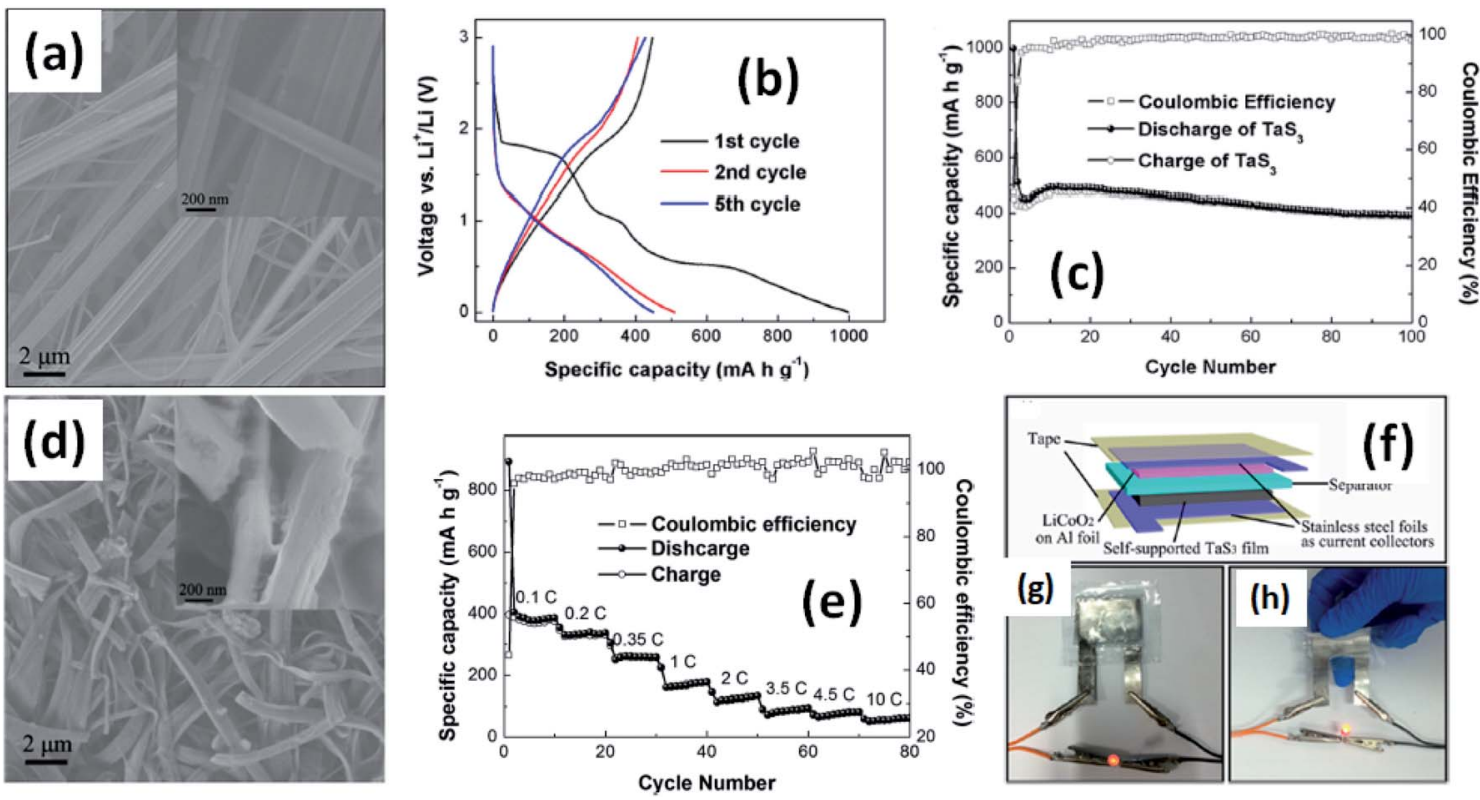

Fig. 18 (a) An SEM image of $\mathrm{TaS}_{3}$ nanowires. (b) Voltage profiles of a lithium-ion battery based on $\mathrm{TaS}_{3}$ nanowires cycled between 0.001 and $3 \mathrm{~V}$ vs. $\mathrm{Li}^{+} / \mathrm{Li}$ at a cycling rate of $0.1 \mathrm{C}$. (c) Capacity and coulombic efficiency as a function of cycle number for a TaS 3 nanowire electrode at a cycling rate of 0.1C. (d) An SEM image of nanowires after long-term cycling tests. (e) Rate performance and coulombic efficiency as a function of cycle number for $\mathrm{TaS}_{3}$ electrodes and as a function of discharge rate $(0.1-10 \mathrm{C})$. ( $\left.\mathrm{f}-\mathrm{h}\right) \mathrm{A}$ schematic diagram of a fabricated flexible lithium-ion battery with photographs showing a glowing LED powered by flat and bent states. Reprinted with permission from ref. 134, copyright: 2015, American Chemical Society.

\subsection{Lithium ion batteries}

Due to their multi-electron processes with high theoretical capacity, $\mathrm{MX}_{3}$-based materials have emerged as potential active materials for lithium- and sodium-ion battery applications. ${ }^{152-155} \mathrm{Wu}$ et al. investigated $\mathrm{Li} / \mathrm{Na}$ adsorption and diffusion in bulk, few-layer, and monolayer $\mathrm{TiS}_{3}$ via studying the phase stability, electron properties, adsorption and dispersion properties, capacity, and plateaus. Charge density and Bader charge analysis studies provided information about the interactions of $\mathrm{Li} / \mathrm{Na}$ with nearby atoms. ${ }^{152}$ The above-mentioned analysis methods also confirmed significant charge transfer from $\mathrm{Li}$ or $\mathrm{Na}$ atoms to surrounding sulphur atoms, and this effect was prominent in monolayer $\mathrm{TiS}_{3}$. Amorphous $\mathrm{TiS}_{3}$, prepared via the ball milling of $\mathrm{TiS}_{2}$ and $\mathrm{S}$, is testified to show a capacity of $400 \mathrm{~mA} \mathrm{~h} \mathrm{~g}{ }^{-1}$ when used as a Li-ion battery electrode material. ${ }^{153}$ Tanibata et al. reported an all-solid-state sodium cell using $\mathrm{TiS}_{3}$ as the active material, which showed a capacity of over $300 \mathrm{~mA} \mathrm{~h} \mathrm{~g}{ }^{-1}$ during the first chargedischarge process. ${ }^{154}$ The capacity of a cell with a $\mathrm{TiS}_{3}$ electrode is reported to be three times higher than that of a cell with $\mathrm{TiS}_{2}$ crystals. Ex situ characterization (XRD and Raman spectroscopy) studies of the electrode materials after long-term cycling tests confirmed that $\mathrm{TiS}_{3}$ maintained its amorphous nature and local structure. Considering the advantages of its metallic nature and the high surface area of $\mathrm{NbSe}_{3}$, Li et al. demonstrated its application as an anode material in lithium-ion batteries. ${ }^{155}$ Pure and $\mathrm{NbSe}_{3}$ nanobelts wrapped with reduced graphene oxide (rGO) were prepared via a chemical approach (Fig. 17ac). ${ }^{155}$ The wrapped rGO utilized strain from $\mathrm{NbSe}_{3}$ structural distortion to suppress impairment induced by volume alterations in the structure of $\mathrm{NbSe}_{3}$ nanobelts during chargedischarge cycles (Fig. 17g). ${ }^{155} \mathrm{NbSe}_{3} @$ rGO showed a discharge capacity of $300 \mathrm{~mA} \mathrm{~h} \mathrm{~g}^{-1}$ after 250 cycles at a current density of $100 \mathrm{~mA} \mathrm{~g}^{-1}$, which was four times greater than that of untainted $\mathrm{NbSe}_{3}$ nanobelts. Li et al. demonstrated self-supported and flexible lithium ion battery anode electrodes based on $\mathrm{TaS}_{3}$ nanowires with a good reversible capacity of $\sim 400 \mathrm{~mA} \mathrm{~h} \mathrm{~g}$ after 100 cycles at $0.1 \mathrm{C}$ with $0.1 \%$ decay (Fig. 18). ${ }^{134}$ Due to the continuous and interconnected nature of the $\mathrm{TaS}_{3}$ nanowires, binder-free and self-supported electrodes could be fabricated, which not only enabled fast electron and ion access but also provided high mechanical flexibility in the fabricated cells.

\subsection{Thermoelectricity}

The thermoelectric effect is mainly a translation between temperature gradients in a material and electronic voltage, and it also works contrariwise. Thermoelectric constituents convert heat into electric power and they have been used to design ecofriendly and sustainable energy sources. ${ }^{156,157}$ The efficiency of thermoelectric materials is estimated based on the figure of merit parameter, $Z T=S^{2} \sigma T / k$, where $S, \sigma, T$, and $k$ are the Seebeck co-efficient, electrical conductivity, absolute temperature, and thermal conductivity, respectively. The thermal conductivity component includes both electronic $\left(k_{\mathrm{e}}\right)$ and phonon (lattice, $k_{\mathrm{l}}$ ) parts. Hence, a proper combination of $S, \sigma$, and $k$ is expected to lead to high-performance thermoelectric materials. Some strategies, such as using low-dimensional structures, alloy defects, band engineering, etc., have been 
employed for this purpose. ${ }^{\mathbf{1 5 8 - 1 6 1}}$ The thermoelectric properties of $\mathrm{MX}_{3}$ materials are reported to show robust anisotropic behaviour in bulk, few-layer, and monolayer form. ${ }^{162}$ Wang et al. reported that the power factor $\left(S^{2} \sigma\right)$ along the $Y$-direction is increased compared to that along the $X$-direction for $\mathrm{ZrS}_{3}{ }^{162}$ Phonon transport findings also demonstrate large anisotropy characteristics induced by unlike scattering from layered $\mathrm{ZrS}_{3}$ along the $X$ and $Z$ directions and different group velocities along the $X$ and $Y$ directions. DFT studies of $\mathrm{ZrSe}_{3}$ monolayers reveal that corrugated conduction levels can result in solid anisotropy of the electric transport characteristics. ${ }^{30}$ It has also been reported that heat transference in $\mathrm{ZrSe}_{3}$ monolayers is completely under-controlled by superficial Se atoms. The large bond lengths of the $\mathrm{Zr}-\mathrm{Se}_{1}$ and $\mathrm{Zr}-\mathrm{Se}_{2}$ chains confine their influence on heat transference and unswervingly lead to low levels of lattice thermal conductivity.

\section{Summary and future directions}

Thorough experimental and theoretical studies of quasi-1D $\mathrm{MX}_{3}$ have shown their wide range of applications in the fields of condensed matter physics and nanotechnology due to their various outstanding electrical, optical, magnetic, and CDW properties, and strong in-plane anisotropy with a quasi-1D nature. The dynamic crystal structures and new fabrication techniques of these materials are well depicted in this work. Also, novel applications based on these materials can be expanded to countless conceivable concepts, which can be viewed perfectly based on the above details.

Weak van der Waals forces of attraction between layers and anisotropy along the chain axis ( $b$-axis) provide $\mathrm{MX}_{3}$ materials with the advantages of both structural (2D layered material and quasi-1D) and electrical (CDW phenomena) properties. In $\mathrm{MX}_{3}$, the chalcogen atoms $(\mathrm{S}, \mathrm{Se}, \mathrm{Te})$ are considered to be electron reservoirs, thus providing exceptional electronic properties, arising from bulk and nanostructured materials. The $\mathrm{M}$ atom presents at the centres of all prismatic chains of $\mathrm{MX}_{6}$, which are linked to each other in an infinite chain manner and run parallel to the $b$-axis. At the base of the prism of $\mathrm{MX}_{3}$, one bond is shorter compared to the others, so more research needs to be carried out in this direction utilizing the advantages of this property, so that new applications of these materials can be explored. Even $\mathrm{TiS}_{3}$ shows strong dichroism in agreement with systematic anisotropy. Similarly, the creation of different types of vacancies in the $\mathrm{TiS}_{3}$ system leads to the induction of a definite magnetic moment. Spin-orbit interactions in the case of $\mathrm{ZrX}_{3}$ materials result in an increase in the energy split from $\mathrm{S}$ to Se atoms. Certain phonon modes undergo a dramatic linewidth reduction near $T_{\mathrm{CDW}}$, representing the strong coupling of phonons with electronic degrees of freedom associated with the CDW for $\mathrm{ZrTe}_{3}$. However, huge attention and focus should be given to the interesting property of $\mathrm{MX}_{3}$, i.e., the $\mathrm{CDW}$, so that the shift in the CDW transition can be well-understood and numerous unexplored applications can be instigated in this direction. High-end techniques and better qualitative practical methods, like solid-state NMR, photon correlation spectroscopy, X-ray diffraction topography (XRT), in situ and ex situ characterization (XRD, SEM, and TEM), muon-spectroscopy, terahertz spectroscopy, etc., can be engaged to study and visualize the LRO pyramid in few-layer or monolayer $\mathrm{MX}_{3}$. The properties are quite poor along the $a$-axis, and they can be tuned via the application of external pressure, strain, etc. More and more focus should be given to studying the anisotropy in the $a$ axis direction, which is somewhat present in some metal tritellurides and will be helpful for outstanding applications in the field of nanotechnology. Therefore, these properties open up the endless possibility for spectacular applications. In contrast, 2D layered material characteristics are somehow not properly shown by the materials due to the quasi-1D nature.

Synthesis approaches for $\mathrm{MX}_{3}$ materials are quite costeffective and environmentally friendly and less timeconsuming in comparison to other 2D material families, like MXenes. These methods include direct chemical reactions, chemical vapour transport (CVT), chemical vapour deposition (CVD), mechanical and chemical exfoliation, etc. Subsequently, other atoms can be intercalated into the layers of $\mathrm{MX}_{3}$ in line with the feeble van der Waals forces. Characterization techniques such as X-ray diffraction (XRD), X-ray photoelectron spectroscopy (XPS), field-emission scanning electron microscopy (FESEM), electron-dispersive X-ray analysis (EDAX), atomic force microscopy (AFM), scanning tunnelling microscopy (STM), transmission electron microscopy (TEM), Raman spectroscopy, UV-visible spectroscopy, and optical microscopy are typical methods used to appraise the properties of $\mathrm{MX}_{3}$ materials. Mostly, CVD and CVT methods are used for producing bulk as well as nanostructured materials with layers of few $\mathrm{mm}$ to nm thickness. As monolayer materials show interesting and research-driven properties, innovative and scalable exfoliation techniques can be used to form thin monolayers.

Most materials are fabricated via taking both precursors at a particular stoichiometry, placing them into a vacuum-sealed ampule, and heating them at a certain temperature for a precise amount of time. Later, after the system cools down, the prepared samples are removed. Via these methods, nanowhiskers, nanoribbons, nanosheets, etc. of $\mathrm{MX}_{3}\left(\mathrm{TiS}_{3}, \mathrm{ZrX}_{3}\right.$, $\mathrm{HfX}_{3}, \mathrm{NbX}_{3}$ ) are prepared, and their characterization is carried out via the above-stated techniques. However, these methods are exceedingly time consuming, and some experiments take 15 days. Therefore, more effective strategies should be implemented for the growth of bulk as well as monolayered $\mathrm{MX}_{3}$. Upgraded CVD and CVT systems with high-end programming for the skillful flow of inert gases $\left(\mathrm{Ar}, \mathrm{H}_{2}, \mathrm{~N}_{2}\right.$, or a mixture of any two) and proper temperature maintaining tools should be prearranged to control the number of layers and their thickness.

$\mathrm{MX}_{3}$ materials show a wide range of applications, e.g., FETs, solar and fuel cells, lithium-ion batteries, photodetectors, photosensors, and thermoelectrics. The higher mobilities and greater $\mathrm{ON} / \mathrm{OFF}$ ratios of $\mathrm{MX}_{3}$-based FETs are worth noting in the present day. $\mathrm{MX}_{3}$ materials possess the advantages of having a low band gap to absorb direct solar energy, and being low-cost, plentiful, and appropriate for photoelectrochemical water splitting, making them suitable candidates for use in solar and fuel cells. The maximum efficiency reached is about 16-20\%. The high photoresponse, fast switching time, and high 
cut-off frequency make $\mathrm{MX}_{3}$ the best material for use in sensors and photodetectors. $\mathrm{MX}_{3}$ can also be used in converting heat into electrical power due to its transport properties. Despite all the merits possessed by $\mathrm{MX}_{3}$, these materials are well behind in terms of real-world applications, like in supercapacitors, sodium-ion batteries, etc. These materials can be fused with carbon-based materials (single-walled and multiwalled carbon nanotubes and rGO) for supercapacitor applications due to their high surface areas. The efficiencies of solar cells can be improved via the incorporation and doping of active elements, which can allow more focus on the real-world applications of $\mathrm{MX}_{3}$. Work should be done to increase the capacities of lithiumion batteries based on $\mathrm{MX}_{3}$ via the strong intercalation of other atoms into the layers of $\mathrm{MX}_{3}$. The photoresponse can be enhanced along with the switching time in photodetectors. $\mathrm{MX}_{3}$ materials can be used for building biosensors, as they are nontoxic and eco-friendly in nature.

This review article highlights the significance of the in-plane anisotropy and quasi-1D nature of $\mathrm{MX}_{3}$ materials, with a complete study of the atomic structures, physical and chemical properties, intrinsic modulations in CDW states, cost- and time-effective synthesis methodologies, and related plausible applications. We contemplate optimistically that this fragment of work on quasi-1D $\mathrm{MX}_{3}$ materials, the evolution of their primary properties, and their related applications will have contemporary significance, leading to novel and fresh research.

\section{Conflicts of interest}

There are no conflicts to declare.

\section{Acknowledgements}

This work was financially supported by the Department of Science and Technology (DST)-SERB Early Career Research project (Grant No. ECR/2017/001850), DST (DST/NM/NT/2019/ 205(G); DST/TDT/SHRI-34/2018), and Karnataka Science and Technology Promotion Society (KSTePS/VGST-RGS-F/2018-19/ GRD No. 829/315).

\section{References}

1 J. Xu, J. Zhang, W. Zhang and C.-S. Lee, Adv. Energy Mater., 2017, 7, 1700571.

2 J. O. Island, A. J. Molina-Mendoza, M. Barawi, R. Biele, E. Flores, J. M. Clamagirand, J. R. Ares, C. Sánchez, H. S. J. van der Zant, R. D'Agosta, I. J. Ferrer and A. Castellanos-Gomez, 2D Materials, 2017, 4, 022003.

3 J. Dai, M. Li and X. C. Zeng, Wiley Interdiscip. Rev.: Comput. Mol. Sci., 2016, 6, 211-222.

4 S. K. Srivastava and B. N. Avasthi, J. Mater. Sci., 1992, 27, 3693-3705.

5 J. Gopalakrishnan and K. S. Nanjundaswamy, Bull. Mater. Sci., 1983, 5, 287-306.

6 J. O. Island, R. Biele, M. Barawi, J. M. Clamagirand, J. R. Ares, C. Sánchez, H. S. J. van der Zant, I. J. Ferrer,
R. D'Agosta and A. Castellanos-Gomez, Sci. Rep., 2016, 6, 1-7.

7 F. Iyikanat, R. T. Senger, F. M. Peeters and H. Sahin, ChemPhysChem, 2016, 17, 3985-3991.

8 R. Sun, Y. Gu, G. Yang, J. Wang, X. Fang, N. Lu, B. Hua and X. Yan, J. Phys. Chem. C, 2019, 123, 7390-7396.

9 J. Kang and L.-W. Wang, Phys. Chem. Chem. Phys., 2016, 18, 14805-14809.

10 J. Dai and X. C. Zeng, Angew. Chem., 2015, 127, 7682-7686. 11 Y. Aierken, D. Çakır and F. M. Peeters, Phys. Chem. Chem. Phys., 2016, 18, 14434-14441.

12 W. Schairer and M. W. Shafer, Phys. Status Solidi A, 1973, 17, 181-184.

13 E. Canadell, Y. Mathey and M. H. Whangbo, J. Am. Chem. Soc., 1988, 110, 104-108.

14 C. Felser, E. W. Finckh, H. Kleinke, F. Rocker and W. Tremel, J. Mater. Chem., 1998, 8, 1787-1798.

15 X. Zhu, B. Lv, F. Wei, Y. Xue, B. Lorentz, L. Deng, Y. Sun and C.-W. Chu, Phys. Rev. B, 2013, 87, 024508.

16 A. J. Molina-Mendoza, M. Barawi, R. Biele, E. Flores, J. R. Ares, C. Sánchez, G. Bollinger, N. Agraït, R. D'Agosta, I. J. Ferrer and A. Castellanos-Gomez, Adv. Electron. Mater., 2015, 1, 1500126.

17 R. Biele, E. Flores, J. R. Ares, C. Sanchez, I. J. Ferrer, G. Rubio-Bollinger, A. Castellanos-Gomez and R. D'Agosta, Nano Res., 2017, 11, 225-232.

18 A. Khatibi, R. H. Godiksen, S. B. Basuvalingam, D. Pellegrino, A. A. Bol, B. Shokri and A. G. Curto, 2D Materials, 2019, 7, 015022.

19 F. Iyikanat, H. Sahin, R. T. Senger and F. M. Peeters, J. Phys. Chem. C, 2015, 119, 10709-10715.

20 J. Kang, H. Sahin, H. D. Ozaydin, R. T. Senger and F. M. Peeters, Phys. Rev. B: Condens. Matter Mater. Phys., 2015, 92, 075413.

21 D. Pacilé, M. Papagno, M. Lavagnini, H. Berger, L. Degiorgi and M. Grioni, Phys. Rev. B: Condens. Matter Mater. Phys., 2007, 76, 155406.

22 A. Ait-Ouali and S. Jandl, Phys. Rev. B: Condens. Matter Mater. Phys., 1996, 53, 9852-9858.

23 A. Pant, E. Torun, B. Chen, S. Bhat, X. Fan, K. Wu, D. P. Wright, F. M. Peeters, E. Soignard, H. Sahin and S. Tongay, Nanoscale, 2016, 8, 16259-16265.

24 W. Kong, C. Bacaksiz, B. Chen, K. Wu, M. Blei, X. Fan, Y. Shen, H. Sahin, D. Wright, D. S. Narang and S. Tongay, Nanoscale, 2017, 9, 4175-4182.

25 X. Wang, K. Wu, M. Blei, Y. Wang, L. Pan, K. Zhao, C. Shan, M. Lei, Y. Cui, B. Chen, D. Wright, W. Hu, S. Tongay and Z. Wei, Adv. Electron. Mater., 2019, 5, 1900419.

26 H. Jin, D. Cheng, J. Li, X. Cao, B. Li, X. Wang, X. Liu and X. Zhao, Solid State Sci., 2011, 13, 1166-1171.

27 A. S. Pawbake, J. O. Island, E. Flores, J. R. Ares, C. Sanchez, I. J. Ferrer, S. R. Jadkar, H. S. J. van der Zant, A. CastellanosGomez and D. J. Late, ACS Appl. Mater. Interfaces, 2015, 7, 24185-24190.

28 K. Wu, E. Torun, H. Sahin, B. Chen, X. Fan, A. Pant, D. Parsons Wright, T. Aoki, F. M. Peeters, E. Soignard and S. Tongay, Nat. Commun., 2016, 7, 1-7. 
29 K. Patel, J. Prajapati, R. Vaidya and S. G. Patel, Bull. Mater. Sci., 2005, 28, 405-410.

30 Z. Zhou, H. Liu, D. Fan, G. Cao and C. Sheng, ACS Appl. Mater. Interfaces, 2018, 10, 37031-37037.

31 M. Li, J. Dai and X. Cheng Zeng, Nanoscale, 2015, 7, 1538515391.

32 Y.-R. Tao, J.-Q. Chen, J.-J. Wu, Y. Wu and X.-C. Wu, J. Alloys Compd., 2016, 658, 6-11.

33 Q. Zhao, Y. Guo, Y. Zhou, Z. Yao, Z. Ren, J. Bai and X. Xu, Nanoscale, 2018, 10, 3547-3555.

34 A. Zwick, M. A. Renucci and A. Kjekshus, J. Phys. C: Solid State Phys., 1980, 13, 5603-5614.

35 P. Poltarak, A. Poltarak, S. Artemkina, T. Podlipskaya, I. Asanov and V. Fedorov, Colloids Surf., A, 2019, 579, 123667.

36 S. L. Gleason, Y. Gim, T. Byrum, A. Kogar, P. Abbamonte, E. Fradkin, G. J. MacDougall, D. J. Van Harlingen, X. Zhu, C. Petrovic and S. L. Cooper, Phys. Rev. B: Condens. Matter Mater. Phys., 2015, 15, 155124.

37 I. G. Gorlova, S. G. Zybtsev, V. Y. Pokrovskii, N. B. Bolotina, I. A. Verin and A. N. Titov, Phys. B, 2012, 407, 1707-1710.

38 T. Ritschel, J. Trinckauf, K. Koepernik, B. Büchner, M. v. Zimmermann, H. Berger, Y. I. Joe, P. Abbamonte and J. Geck, Nat. Phys., 2015, 11, 328-331.

39 H. Feng, Z. Xu, J. Zhuang, L. Wang, Y. Liu, X. Xu, L. Song, W. Hao and Y. Du, Adv. Funct. Mater., 2019, 29, 1900367.

40 S. Takahashi, T. Sambongi, J. W. Brill and W. Roark, Solid State Commun., 1984, 49, 1031-1034.

41 H. Nakajima, K. Nomura and T. Sambongi, Physica $B+C$, 2002, 143, 240-242.

42 M. Hoesch, X. Cui, K. Shimada, C. Battaglia, S. Fujimori and H. Berger, Phys. Rev. B: Condens. Matter Mater. Phys., 2009, 80, 075423.

43 D. J. Eaglesham, J. W. Steeds and J. A. Wilson, J. Phys. C: Solid State Phys., 1984, 17, L697-L698.

44 Y. Hu, F. Zheng, X. Ren, J. Feng and Y. Li, Phys. Rev. B: Condens. Matter Mater. Phys., 2015, 91, 144502.

45 K. Yamaya, S. Takayanagi and S. Tanda, Phys. Rev. B: Condens. Matter Mater. Phys., 2012, 85, 184513.

46 M. Hoesch, A. Bosak, D. Chernyshov, H. Berger and M. Krisch, Phys. Rev. Lett., 2009, 102, 086402.

47 R. Yomo, K. Yamaya, M. Abliz, M. Hedo and Y. Uwatoko, Phys. Rev. B: Condens. Matter Mater. Phys., 2005, 71, 132508.

48 M. Hoesch, G. Garbarino, C. Battaglia, P. Aebi and H. Berger, Phys. Rev. B, 2016, 93, 125102.

49 X. Zhu, W. Ning, L. Li, L. Ling, R. Zhang, J. Zhang, K. Wang, Y. Liu, L. Pi, Y. Ma, H. Du, M. Tian, Y. Sun, C. Petrovic and Y. Zhang, Sci. Rep., 2016, 6, 26974.

50 C. S. Yadav and P. L. Paulose, J. Phys.: Condens. Matter, 2012, 24, 235702.

51 J. Li, J. Peng, S. Zhang and G. Chen, Phys. Rev. B, 2017, 96, 174510.

52 T. Ikari, R. Provencher, S. Jandl and M. Aubin, Solid State Commun., 1983, 45, 113-116.

53 S. Lai and Y. Du, Materials, 2019, 12, 3501.
54 V. Y. Pokrovskii, S. G. Zybtsev, M. V. Nikitin, I. G. Gorlova, V. F. Nasretdinova and S. V. Zaitsev-Zotov, Phys.-Usp., 2013, 56, 29-48.

55 M. A. Bloodgood, P. Wei, E. Aytan, K. N. Bozhilov, A. A. Balandin and T. T. Salguero, APL Mater., 2018, 6, 026602.

56 F. Jellinek, G. Brauer and H. Müller, Nature, 1960, 185, 376377.

57 J. Rijnsdorp and F. Jellinek, J. Solid State Chem., 1978, 25, 325-328.

58 D. W. Bullett, J. Solid State Chem., 1980, 33, 13-16.

59 M. E. Itkis, F. Y. Nad', S. V. Zaitsev-Zotov and F. Lévy, Solid State Commun., 1989, 71, 895-898.

60 M. E. Itkis, F. Y. Nad' and F. Levy, Synth. Met., 1991, 43, 3969-3972.

61 Z. Z. Wang, P. Monceau, H. Salva, C. Roucau, L. Guemas and A. Meerschaut, Phys. Rev. B: Condens. Matter Mater. Phys., 1989, 40, 11589-11593.

62 J. W. Brill, H. Zhang and J. Nichols, Phys. B, 2012, 407, 17371740.

63 T. Cornelissens, G. Van Tendeloo, J. Van Landuyt and S. Amelinckx, Phys. Status Solidi A, 1978, 48, K5-K9.

64 E. Zupanič, H. J. P. van Midden, M. A. van Midden, S. Šturm, E. Tchernychova, V. Y. Pokrovskii, S. G. Zybtsev, V. F. Nasretdinova, S. V. Zaitsev-Zotov, W. T. Chen, W. W. Pai, J. C. Bennett and A. Prodan, Phys. Rev. B, 2018, 98, 174113.

65 S. Kikkawa, N. Ogawa, M. Koizumi and Y. Onuki, J. Solid State Chem., 1982, 41, 315-322.

66 S. G. Zybtsev, V. Y. Pokrovskii, V. F. Nasretdinova, S. V. Zaitsev-Zotov, V. V. Pavlovskiy, A. B. Odobesco, W. W. Pai, M.-W. Chu, Y. G. Lin, E. Zupanič, H. J. P. van Midden, S. Šturm, E. Tchernychova, A. Prodan, J. C. Bennett, I. R. Mukhamedshin, O. V. Chernysheva, A. P. Menushenkov, V. B. Loginov, B. A. Loginov, A. N. Titov and M. Abdel-Hafiez, Phys. Rev. B, 2017, 95, 035110.

67 A. Zettl, C. M. Jackson, A. Janossy, G. Grüner, A. Jacobsen and A. H. Thompson, Solid State Commun., 1982, 43, 345347.

68 A. Meerschaut and J. Rouxel, J. Less-Common Met., 1975, 39, 197-203.

69 P. Monçeau, N. P. Ong, A. M. Portis, A. Meerschaut and J. Rouxel, Phys. Rev. Lett., 1976, 37, 602-606.

70 J. L. Hodeau, M. Marezio, C. Roucau, R. Ayroles, A. Meerschaut, J. Rouxel and P. Monceau, J. Phys. C: Solid State Phys., 1978, 11, 4117-4134.

71 R. M. Fleming, D. E. Moncton and D. B. McWhan, Phys. Rev. B: Solid State, 1978, 18, 5560-5563.

72 Y. S. Hor, Z. L. Xiao, U. Welp, Y. Ito, J. F. Mitchell, R. E. Cook, W. K. Kwok and G. W. Crabtree, Nano Lett., 2005, 5, 397-401.

73 R. E. Thorne, W. G. Lyons, J. W. Lyding, J. R. Tucker and J. Bardeen, Phys. Rev. B: Condens. Matter Mater. Phys., 1987, 35, 6348-6359.

74 C. Brun, Z.-Z. Wang and P. Monceau, Phys. Rev. B: Condens. Matter Mater. Phys., 2009, 80, 045423. 
75 A. Meerschaut, L. Guemas and J. Rouxel, J. Solid State Chem., 1981, 36, 118-123.

76 F. Jellinek, J. Less-Common Met., 1962, 4, 9-15.

77 E. Bjerkelund and A. Kjekshus, Z. Anorg. Allg. Chem., 1964, 328, 235-242.

78 T. A. Empante, A. Martinez, M. Wurch, Y. Zhu, A. K. Geremew, K. Yamaguchi, M. Isarraraz, S. Rumyantsev, E. J. Reed, A. A. Balandin and L. Bartels, Nano Lett., 2019, 19, 4355-4361.

79 T. Sambongi, M. Yamamoto, K. Tsutsumi, Y. Shiozaki, K. Yamaya and Y. Abe, J. Phys. Soc. Jpn., 1977, 42, 14211422.

80 E. Bjerkelund, A. Kjekshus, G. Widmark, P. H. Nielsen, B. Sjöberg and E. Larsen, Acta Chem. Scand., 1965, 19, 701-710.

81 E. M. Dizhur, M. A. Il'ina and S. V. Zaitsev-Zotov, JETP Lett., 2007, 86, 132-134.

82 V. E. Fedorov, S. B. Artemkina, E. D. Grayfer, N. G. Naumov, Y. V. Mironov, A. I. Bulavchenko, V. I. Zaikovskii, I. V. Antonova, A. I. Komonov and M. V. Medvedev, J. Mater. Chem. C, 2014, 2, 5479-5486.

83 S. B. Artemkina, E. D. Grayfer, M. N. Kozlova, S. G. Kozlova, M. R. Ryzhikov, I. R. Shein and V. E. Fedorov, Spectrochim. Acta, Part A, 2017, 179, 46-50.

84 K. Wu, M. Blei, B. Chen, L. Liu, H. Cai, C. Brayfield, D. Wright, H. Zhuang and S. Tongay, Adv. Mater., 2020, 32, 2000018.

85 H. M. McConnell and R. Lynden-Bell, J. Chem. Phys., 1962, 36, 2393-2397.

86 C. Sourisseau, R. Cavagnat, M. Fouassier and P. Maraval, J. Raman Spectrosc., 1990, 21, 337-349.

87 J. Chaussy, P. Haen, J. C. Lasjaunias, P. Monceau, G. Waysand, A. Waintal, A. Meerschaut, P. Molinié and J. Rouxel, Solid State Commun., 1976, 20, 759-763.

88 S. van Smaalen, J. L. de Boer, A. Meetsma, H. Graafsma, H.-S. Sheu, A. Darovskikh, P. Coppens and F. Levy, Phys. Rev. B: Condens. Matter Mater. Phys., 1992, 45, 3103-3106.

89 H. Shi, H. Yang, H. Tian, Z. Wang, Y. Qin, Y. Song, M. Luo, W. Wang and J. Li, J. Nanosci. Nanotechnol., 2013, 13, 41234128.

90 M. Ido, Y. Okayama, T. Ijiri and Y. Okajima, J. Phys. Soc. Jpn., 1990, 59, 1341-1347.

91 Y. I. Latyshev, O. Laborde, P. Monceau and S. Klaumünzer, Phys. Rev. Lett., 1997, 78, 919-922.

92 L. Yang, Y. Tao, J. Liu, C. Liu, Q. Zhang, M. Akter, Y. Zhao, T. T. Xu, Y. Xu, Z. Mao, Y. Chen and D. Li, Nano Lett., 2018, 19, 415-421.

93 N. P. Ong and P. Monceau, Solid State Commun., 1978, 26, 487-491.

94 C. Roucau, R. Ayroles, P. Monceau, L. Guemas, A. Meerschaut and J. Rouxel, Phys. Status Solidi A, 1980, 62, 483-493.

95 S. Sugai, Phys. Rev. B: Condens. Matter Mater. Phys., 1984, 29, 953-965.

96 S. G. Zybtsev and V. Y. Pokrovskii, Phys. Rev. B, 2016, 94, 115140.
97 K. Inagaki, T. Toshima and S. Tanda, J. Phys. Chem. Solids, 2005, 66, 1563-1566.

98 J. Nichols, D. Dominko, L. Ladino, J. Zhou and J. W. Brill, Phys. Rev. B: Condens. Matter Mater. Phys., 2009, 79, 241110.

99 S. Hoen, B. Burk, A. Zettl and M. Inui, Phys. Rev. B: Condens. Matter Mater. Phys., 1992, 46, 1874-1877.

100 A. V. Golovnya, V. Y. Pokrovskii and P. M. Shadrin, Phys. Rev. Lett., 2002, 88, 246401.

101 V. Pokrovskii, S. Zybtsev and I. Gorlova, Phys. Rev. Lett., 2007, 98, 206404.

102 J. Nichols, C. Sandamali Weerasooriya and J. W. Brill, J. Phys.: Condens. Matter, 2010, 22, 334224.

103 K. Inagaki, T. Matsuura, M. Tsubota, S. Uji, T. Honma and S. Tanda, Phys. Rev. B, 2016, 93, 075423.

104 K. E. Farley, Z. Shi, G. Sambandamurthy and S. Banerjee, Phys. Chem. Chem. Phys., 2015, 17, 18374-18379.

105 K. Wu, B. Chen, H. Cai, M. Blei, J. Bennett, S. Yang, D. Wright, Y. Shen and S. Tongay, J. Phys. Chem. C, 2017, 121, 28187-28193.

106 S. Sridhar, D. Reagor and G. Gruner, Phys. Rev. Lett., 1985, 55, 1196-1199.

107 B. J. Kim, B. J. Jeong, S. Oh, S. Chae, K. H. Choi, T. Nasir, S. H. Lee, H. K. Lim, I. J. Choi, M.-K. Hong, H. K. Yu, J.-H. Lee and J.-Y. Choi, Materials, 2019, 12, 2462.

108 M. A. Stolyarov, G. Liu, M. A. Bloodgood, E. Aytan, C. Jiang, R. Samnakay, T. T. Salguero, D. L. Nika, S. L. Rumyantsev, M. S. Shur, K. N. Bozhilov and A. A. Balandin, Nanoscale, 2016, 8, 15774-15782.

109 G. Kumagai, T. Matsuura, K. Ichimura, K. Yamaya, K. Inagaki and S. Tanda, Phys. Rev. B: Condens. Matter Mater. Phys., 2010, 81, 184506.

110 G. Liu, S. Rumyantsev, M. A. Bloodgood, T. T. Salguero, M. Shur and A. A. Balandin, Nano Lett., 2016, 17, 377-383.

111 A. Nomura, K. Yamaya, S. Takayanagi, K. Ichimura, T. Matsuura and S. Tanda, Europhys. Lett., 2017, 119, 17005.

112 Y.-H. Chang, W. Zhang, Y. Zhu, Y. Han, J. Pu, J.-K. Chang, W.-T. Hsu, J.-K. Huang, C.-L. Hsu, M.-H. Chiu, T. Takenobu, H. Li, C.-I. Wu, W.-H. Chang, A. T. S. Wee and L.-J. Li, ACS Nano, 2014, 8, 8582-8590.

113 J. Zhou, J. Lin, X. Huang, Y. Zhou, Y. Chen, J. Xia, H. Wang, Y. Xie, H. Yu, J. Lei, D. Wu, F. Liu, Q. Fu, Q. Zeng, C.-H. Hsu, C. Yang, L. Lu, T. Yu, Z. Shen, H. Lin, B. I. Yakobson, Q. Liu, K. Suenaga, G. Liu and Z. Liu, Nature, 2018, 556, 355-359.

114 H. Li, Y. Li, A. Aljarb, Y. Shi and L.-J. Li, Chem. Rev., 2017, 118, 6134-6150.

115 Z. Zhang, P. Yang, M. Hong, S. Jiang, G. Zhao, J. Shi, Q. Xie and Y. Zhang, Nanotechnology, 2019, 30, 182002.

116 S. Tongay, W. Fan, J. Kang, J. Park, U. Koldemir, J. Suh, D. S. Narang, K. Liu, J. Ji, J. Li, R. Sinclair and J. Wu, Nano Lett., 2014, 14, 3185-3190.

117 S. Wang, X. Wang and J. H. Warner, ACS Nano, 2015, 9, 5246-5254.

118 K. M. McCreary, A. T. Hanbicki, J. T. Robinson, E. Cobas, J. C. Culbertson, A. L. Friedman, G. G. Jernigan and B. T. Jonker, Adv. Funct. Mater., 2014, 24, 6449-6454.

119 Z. Chen, Y. Qi, X. Chen, Y. Zhang and Z. Liu, Adv. Mater., 2018, 31, 1803639. 
120 P. Chen, Z. Zhang, X. Duan and X. Duan, Chem. Soc. Rev., 2018, 47, 3129-3151.

121 H. Haraldsen, A. Kjekshus, E. Røst, A. Steffensen and J. Munch-Petersen, Acta Chem. Scand., 1963, 17, 1283-1292.

122 L. Brattås, A. Kjekshus, J. Krogh-Moe, J. Songstad and Å. Pilotti, Acta Chem. Scand., 1972, 26, 3441-3449.

123 S. Kikkawa, M. Koizumi, S. Yamanaka, Y. Onuki and S. Tanuma, Phys. Status Solidi A, 1980, 61, K55-K57.

124 A. Lipatov, P. M. Wilson, M. Shekhirev, J. D. Teeter, R. Netusil and A. Sinitskii, Nanoscale, 2015, 7, 12291-12296.

125 J. O. Island, M. Buscema, M. Barawi, J. M. Clamagirand, J. R. Ares, C. Sánchez, I. J. Ferrer, G. A. Steele, H. S. J. van der Zant and A. Castellanos-Gomez, Adv. Opt. Mater., 2014, 2, 641-645.

126 J. O. Island, M. Barawi, R. Biele, A. Almazán, J. M. Clamagirand, J. R. Ares, C. Sánchez, H. S. J. van der Zant, J. V. Álvarez, R. D'Agosta, I. J. Ferrer and A. Castellanos-Gomez, Adv. Mater., 2015, 27, 2595-2601.

127 H. S. W. Chang and D. M. Schleich, J. Solid State Chem., 1992, 100, 62-70.

128 H. Yi, S. J. Gilbert, A. Lipatov, A. Sinitskii, J. Avila, J. Abourahma, T. Komesu, M. C. Asensio and P. A. Dowben, J. Phys.: Condens. Matter, 2020, 32, $29 \mathrm{LT01.}$

129 W.-W. Xiong, J.-Q. Chen, X.-C. Wu and J.-J. Zhu, J. Mater. Chem. C, 2015, 3, 1929-1934.

130 X. Yu, X. Wen, W. Zhang, L. Yang, H. Wu, X. Lou, Z. Xie, Y. Liu and H. Chang, CrystEngComm, 2019, 21, 5586-5594.

131 T. Pham, S. Oh, P. Stetz, S. Onishi, C. Kisielowski, M. L. Cohen and A. Zettl, Science, 2018, 361, 263-266.

132 X. Wu, Y. Tao, Y. Hu, Y. Song, Z. Hu, J. Zhu and L. Dong, Nanotechnology, 2005, 17, 201-205.

133 C. C. Mayorga-Martinez, Z. Sofer, J. Luxa, Š. Huber, D. Sedmidubský, P. Brázda, L. Palatinus, M. Mikulics, P. Lazar, R. Medlín and M. Pumera, ACS Nano, 2017, 12, 464-473.

134 W. Li, L. Yang, J. Wang, B. Xiang and Y. Yu, ACS Appl. Mater. Interfaces, 2015, 7, 5629-5633.

135 S. Kikkawa, K. Shinya and M. Koizumi, J. Solid State Chem., 1982, 41, 323-328.

136 E. Canadell, I. E. I. Rachidi, J. P. Pouget, P. Gressier, A. Meerschaut, J. Rouxel, D. Jung, M. Evain and M. H. Whangbo, Inorg. Chem., 1990, 29, 1401-1407.

137 T. Lu, S. Dong, C. Zhang, L. Zhang and G. Cui, Coord. Chem. Rev., 2017, 332, 75-99.

138 Z. Lei, J. Zhan, L. Tang, Y. Zhang and Y. Wang, Adv. Energy Mater., 2018, 8, 1703482.

139 J. Xie, R. Wang, J. Bao, X. Zhang, H. Zhang, S. Li and Y. Xie, Inorg. Chem. Front., 2014, 1, 751-756.

140 R. R. Chianelli and M. B. Dines, Inorg. Chem., 1975, 14, 2417-2421.

141 M. Randle, A. Lipatov, A. Kumar, C.-P. Kwan, J. Nathawat, B. Barut, S. Yin, K. He, N. Arabchigavkani, R. Dixit, T. Komesu, J. Avila, M. C. Asensio, P. A. Dowben,
A. Sinitskii, U. Singisetti and J. P. Bird, ACS Nano, 2018, 13, 803-811.

142 W.-W. Xiong, J.-Q. Chen, X.-C. Wu and J.-J. Zhu, J. Mater. Chem. C, 2014, 2, 7392-7395.

143 X. Chen, S. Shen, L. Guo and S. S. Mao, Chem. Rev., 2010, 110, 6503-6570.

144 M. Barawi, E. Flores, I. J. Ferrer, J. R. Ares and C. Sánchez, J. Mater. Chem. A, 2015, 3, 7959-7965.

145 E. Flores, J. R. Ares, I. J. Ferrer and C. Sánchez, Phys. Status Solidi RRL, 2016, 10, 802-806.

146 R. Ahammed, A. Rawat, N. Jena, Dimple, M. K. Mohanta and A. De Sarkar, Appl. Surf. Sci., 2020, 499, 143894.

147 P. He, X.-X. Wang, Y.-Z. Cai, J.-C. Shu, Q.-L. Zhao, J. Yuan and M.-S. Cao, Nanoscale, 2019, 11, 6080-6088.

148 R. Späh, M. Lux-Steiner, E. Bucher and S. Wagner, Appl. Phys. Lett., 1984, 45, 744-745.

149 Y.-R. Tao, X.-C. Wu and W.-W. Xiong, Small, 2014, 10, 49054911.

150 Y.-Q. Wang, X. Wu, Y.-L. Wang, Y. Shao, T. Lei, J.-O. Wang, S.-Y. Zhu, H. Guo, L.-X. Zhao, G.-F. Chen, S. Nie, H.-M. Weng, K. Ibrahim, X. Dai, Z. Fang and H.-J. Gao, Adv. Mater., 2016, 28, 5013-5017.

151 W. Wu, Y. Wang, Y. Niu, P. Wang, M. Chen, J. Sun, N. Wang, D. $\mathrm{Wu}$ and Z. Zhao, ACS Appl. Mater. Interfaces, 2020, 12, 14165-14173.

152 J. Wu, D. Wang, H. Liu, W.-M. Lau and L.-M. Liu, RSC Adv., 2015, 5, 21455-21463.

153 A. Hayashi, T. Matsuyama, A. Sakuda and M. Tatsumisago, Chem. Lett., 2012, 41, 886-888.

154 N. Tanibata, T. Matsuyama, A. Hayashi and M. Tatsumisago, J. Power Sources, 2015, 275, 284-287.

155 J. Li, Q. Sun, Z. Wang, J. Xiang, B. Zhao, Y. Qu and B. Xiang, Appl. Surf. Sci., 2017, 412, 113-120.

156 K. Biswas, J. He, I. D. Blum, C.-I. Wu, T. P. Hogan, D. N. Seidman, V. P. Dravid and M. G. Kanatzidis, Nature, 2012, 489, 414-418.

157 A. Banik, S. Roychowdhury and K. Biswas, Chem. Commun., 2018, 54, 6573-6590.

158 J. Sun, H. Shi, T. Siegrist and D. J. Singh, Appl. Phys. Lett., 2015, 107, 153902.

159 J. Shen, X. Zhang, S. Lin, J. Li, Z. Chen, W. Li and Y. Pei, J. Mater. Chem. A, 2016, 4, 15464-15470.

160 Y. Pei, X. Shi, A. LaLonde, H. Wang, L. Chen and G. J. Snyder, Nature, 2011, 473, 66-69.

161 S. Lin, W. Li, X. Zhang, J. Li, Z. Chen and Y. Pei, Inorg. Chem. Front., 2017, 4, 1066-1072.

162 C. Wang, C. Zheng and G. Gao, J. Phys. Chem. C, 2020, 124, 6536-6543.

163 D. Zhao, S. Budhi, A. Rodriguez and R. T. Koodali, Int. J. Hydrogen Energy, 2010, 35, 5276-5283.

164 D. W. Bullett, J. Phys. C: Solid State Phys., 1979, 12, 277-281.

165 M. Abdulsalam and D. P. Joubert, Phys. Status Solidi B, 2016, 253, 868-874.

166 F. Lévy and H. Berger, J. Cryst. Growth, 1983, 61, 61-68. 